\title{
Hedgehog Signalling Modulates Glial Proteostasis and Lifespan.
}

Andrew Rallis ${ }^{1}$, Juan A. Navarro ${ }^{2}$, Mathias Rass ${ }^{2}$, Amélie $\mathrm{Hu}^{3}$, Serge Birman ${ }^{3}$, Stephan Schneuwly ${ }^{2}$ and Pascal $P$. Thérond ${ }^{1 *}$.

${ }^{1}$ Université Côte d'Azur, CNRS, Inserm, iBV, France

${ }^{2}$ Department of Developmental Biology, Institute of Zoology, Universitaetsstr. 31, University of Regensburg, 93040 Regensburg, Germany.

${ }^{3}$ Genes Circuits Rhythms and Neuropathology, Brain Plasticity Unit, ESPCI Paris, CNRS, Labex MemoLife, PSL Research University, 10 rue Vauquelin, 75005 Paris, France

* Corresponding author: therond@unice.fr

\section{SUMMARY}

The conserved Hedgehog ( $\mathrm{Hh}$ ) signaling pathway has a well-established role in animal development, however its function during adulthood remains largely unknown. Here, we investigated whether the $\mathrm{Hh}$ signaling pathway is active during adult life in Drosophila melanogaster and uncovered a protective function for Hh signaling in coordinating correct proteostasis in glial cells. Adult-specific depletion of Hh reduces lifespan, locomotor activity and dopaminergic neuron integrity. Conversely, increased expression of $\mathrm{Hh}$ extends lifespan and improves fitness. Moreover, Hh pathway activation in glia rescues the lifespan and age-associated defects of hedgehog $(h h)$ mutants. At the molecular level, the Hh pathway regulates downstream chaperones, principally $h s p 40$ and $h s p 68$, whose overexpression in glial cells rescues the shortened lifespan and proteostasis defects of hh mutants. Finally, we demonstrate the protective ability of Hh signalling in a Drosophila Alzheimer's disease model expressing human Amyloid Beta $(\mathrm{A} \beta 1-42)$ in the glia. Overall, we propose that $\mathrm{Hh}$ signalling is requisite for lifespan determination and correct proteostasis in glial cells and may have potential in ameliorating a wide range of degenerative diseases.

\section{INTRODUCTION}

The Hedgehog $(\mathrm{Hh})$ signalling pathway is an evolutionarily conserved module that organizes animal patterning and is essential for determining cell identity and the final body plan (Briscoe and Thérond., 2013). Hh and components of its pathway were initially 
discovered in Drosophila and highly conserved members are present in the vast majority of metazoa including in mammals, which possess three Hh paralogs, the most well studied being Sonic Hedgehog (Shh; Ingham, 2018). In human, abnormally sustained activation of the Shh pathway in the adult is critical in the initiation and growth of many tumors (Beachy et al., 2004; Curran, 2018). Although the developmental facets of Hh signalling have been well established, the role of $\mathrm{Hh}$ signalling in adult homeostasis has been difficult to evaluate due to the vital function of the Hh pathway during development.

Emerging lines of evidence suggest that Shh has adult specific functions in the nervous system (Petrova and Joyner, 2014). For instance, in mice, Shh regulates the proliferation of neural stem cells throughout adult life (Lai et al., 2003; Palma et al., 2005; Ahn and Joyner, 2005; Ferent et al., 2014; Yao et al., 2016). Shh signalling also has been demonstrated to act as a modulator of brain plasticity and nerve regeneration (Yao et al., 2016). Furthermore, Shh signaling is elevated in different CNS injury models, such as stroke, spinal cord injury and stab wound (Jin et al., 2015; Amankulor et al., 2009; Bambakidis et al., 2010). In line with the above, treatment with Shh or Shh agonists has shown to improve neurological function and promote neuroregeneration (Chechneva et al., 2014; Huang et al., 2013; Bambakidis et al., 2012; Thomas et al., 2018) whereas depletion of Shh signalling components exacerbated the neuropathology following cerebral ischemia and nerve injury (Ji et al., 2012; Martinez et al., 2015).

There is accumulating evidence that Shh signalling utilises a neuron-glia communication mode to coordinate homeostasis in the post-natal brain. Under physiological conditions, Shh is predominantly released by neurons (Gonzalez Reyes et al., 2012; Eitan et al., 2016; Okuda et al., 2016) and glial cells have been shown, in vitro, to be highly responsive to Shh signalling (Ugbode et al., 2017; Okuda et al., 2016). Furthermore, Shh signaling is active in vivo in the adult mouse forebrain (Garcia et al., 2010) in which signaling is activated in a discrete population of glial cells that respond to Shh secreted from neurons (Garcia et al., 2010, Farmer et al., 2016). Importantly, activating Shh signaling in glial cells has been demonstrated to be neuroprotective. For example, in vitro, Shh-stimulated mouse cortical astrocytes protect co-cultured neurons 
from kainite (KA)-induced cell death (Ugbode et al., 2017). Furthermore, in neurodegeneration mouse models induced by intraperitoneal injection of KA, or surgical brain injury, Shh signaling in glia regulates proliferation of astrocytes and microglia (Sirko et al., 2013; Pitter et al, 2014).

Due to its role as a neuroprotective factor, it is hypothesised that Shh may be implicated in alleviating age-related neurodegenerative disease (Zhang et al., 2014; Yao et al., 2017). For instance in cultured rat hippocampal neurons, Shh signalling confers protection against neurotoxins such as the Amyloid beta peptide (A $\beta$ 1-42; Yao et al., 2017). However the mechanism by which Shh exerts its protective function in a live organism has not been explored. In Alzheimer's disease, accumulation of A $\beta 1-42$ has been demonstated to precede the formation of amyloid plaques, and the deposition of amyloid plaques correlates with an increase in age-related cognitive dysfunction (D'Andrea et al., 2001; Rodrigue et al, 2009). A $\beta$ 1-42 has a high propensity to aggregate and there is a large body of evidence that chaperones prevent deleterious misfolding and aggregation of Amyloid (Evans et al, 2006; Magrané et al, 2004). In fact, the Hsp70 chaperone and its partner Hsp40 have been demonstrated to act synergistically to inhibit the early stages of $A \beta$ 1-42 aggregation in vitro (Evans et al, 2006). Moreover in rat primary neuronal cultures seeded with $A \beta 1-42, h s p 70$ overexpression improves cell viability (Magrané et al, 2004). Based on previous studies is possible that Shh prevents accumulation of $A \beta$ 1-42 aggregates, however this has yet to be evaluated.

Here we utilize Drosophila melanogaster to evaluate the function of the $\mathrm{Hh}$ pathway at the physiological and cellular level during adult life. This model enables us to address the tissue-specific functional role of the Hh signaling pathway in the adult brain. We identify a new role for Hh signalling in adult life span and glial proteostasis and uncover the mechanism by which $\mathrm{Hh}$ prevents accumulation of protein aggregates. We show that increasing $\mathrm{Hh}$ levels promotes lifespan extension, whereas depletion of $\mathrm{Hh}$ activity during adult life reduces lifespan. We further demonstrate that specific activation of the Hh pathway in adult glia potently rescues the shortened lifespan, locomotor and dopaminergic neuron defects observed in hedgehog $(h h)$ mutants. We found that proteostasis in glial cells is affected in absence of Hh activity and identified two highly 
conserved chaperones $h s p 40$ and $h s p 68$ (a member of the Hsp70 protein family) that are regulated by the Hh pathway. Their overexpression potently rescues proteostasis defects in the glia of $h h$ mutant animals and rescues $h h$ mutant reduced lifespan. As proof of concept we also demonstrate that activation of the Hh pathway and one of its downstream chaperone targets in the glia is able to rescue the deleterious effects of expression of human Amyloid Beta (A $\beta$ 1-42) in an in vivo Alzheimer's disease model. The work described here makes a significant contribution to our understanding of the role of the $\mathrm{Hh}$ morphogen during adulthood: it identifies for the first time a protective role for Hh on glia proteostasis, and dissects its mechanism of action, showing that Hh operates through the regulation of highly conserved chaperones.

\section{RESULTS}

The Hh signaling pathway is present in glial cells of the adult brain Here, we address the role of Hh signaling in the adult brain of Drosophila melanogaster in which it has not yet been studied. To do so, we first investigated the pattern of expression of Hh signalling components, in particular the Smoothened (Smo) serpentine protein and the transcription factor $\mathrm{Ci} / \mathrm{Gli}$ which is able to activate the expression of target genes (Fig. 1a-d). For this, we utilized the Crispr/Cas9 genome editing and generated a new Ci-Trojan Gal4 line which represents the full Ci expression pattern and, together with a second independent system ( $\mathrm{C} i$-LexA line), found that $\mathrm{C} i$ is predominantly expressed in glial cells in the adult fly brain (Fig. 1a, b and Fig. S1a). Immunofluorescent analysis using antibodies against Smo and $\mathrm{Ci}$ was also performed and revealed a strong localization of both components in glia (Fig. 1c-d). RNAi-depletion of Smo and $\mathrm{Ci}$ in glia reduced immunoreactivity for both proteins, supporting specificity of the stainings (Fig. S1b-e). These findings are consistent with a recent single cell transcriptome analysis of the Drosophila and human adult brain identifying Smo and Ci/Gli expression in glia (Davie et al., 2018, Lake et al, 2018).

Knowing that the Hh pathway is present in adult glial cells, we further examined the pattern of expression of $\mathrm{Hh}$ in the adult brain. Using two different $h h-\mathrm{Gal} 4$ reporter lines, inserted at two different positions in the $h h$ genomic locus, we found that $h h$ is expressed in the adult brain in approximately 10-15 neurons per hemisphere that are 
positive for the pan-neuronal marker Elav, and negative for the glia-specific marker Repo (Fig. 1e-f and S1f-g). Using a third reporter line for $h h$-expressing cells - the $h h$ dsRed enhancer trap (Akimoto et al., 2005) - we identified glutamatergic and GABAergic neurons that are positive for the hh-expressing cells (Fig. S1h-j). This shows that both subtypes of neurons produce $\mathrm{Hh}$, in agreement with recent single cell transcriptome analysis of the Drosophila adult brain (Croset et al., 2018; Davie et al., 2018) and with an equivalent study on Shh in the adult human brain (Lake et al, 2018).

Hh signalling is needed to maintain Dopaminergic neuron viability in the adult brain To investigate the functional relevance of $\mathrm{Hh}$ signalling in adult, we specifically depleted $\mathrm{Hh}$ in the entire organism using a $h h$ temperature sensitive allele $\left(h h^{t s}\right)$. This is a null allele for $h h$ at non-permissive temperature $\left(29^{\circ} \mathrm{C}\right)$ and, after $24 \mathrm{~h}$ at this temperature, no more Hh staining or activity can be detected in homozygote mutant animals (Ranieri et al., 2012; Palm et al., 2010). To avoid any patterning defect due to Hh developmental function, $h h^{\text {ts }}$ flies were raised at permissive temperature $\left(18^{\circ} \mathrm{C}\right)$ and transferred to nonpermissive temperature upon eclosure (Methods). Indeed, these flies do not exhibit any noticeable patterning defect (Strigini et., 1997). With this set up, we investigated whether $\mathrm{Hh}$ is required to maintain neuronal homeostasis in the adult brain. To explore this, we focused on dopaminergic (DA) neurons known to be highly susceptible to brain homeostasis defects, and whose cell number, neuronal projection and secretion of dopamine can be measured and quantified accurately (Friggi-Grelin et al., 2003; Navarro et al., 2014).

Nine DA clusters are present in the Drosophila adult brain, among which the protocerebral anterior medial - PAM - cluster, that is part of the circuit regulating climbing activity (Riemensperger et al., 2013). Strikingly, using anti-Tyrosine hydroxylase (TH, a functional marker for DA neurons) immunostaining (Friggi-Grelin et al., 2003), we observed a reduced number of DA neurons in the PAM cluster of aged $h$ h mutant adult brains (Fig. 1g-h and j) whereas no change in DA neuron number was detected in young hh mutant flies (Fig. 1i, S2f-g). These defects suggest that overall DA neuron volume in the adult brain might be affected in absence of $\mathrm{Hh}$. Indeed, we found that, during adulthood, hh mutant brains globally showed an abnormally low level of TH staining (Fig. 
$1 \mathrm{k}-0$, Table S1) as well as an overall reduction of the mRNA levels of the neuronal specific form of $\mathrm{TH}(\mathrm{nTH})$ and a decrease in brain dopamine levels (Fig. 3n-o, compare green and blue bars). In an alternative approach, we quantified DA neuron volume network by labelling their cell membrane with the mCD8-GFP reporter transgene and found similar results (Fig. S2a-e). To do so, we used the Ddc-Gal4 driver which targets a subset of DA neurons including the entire PAM cluster (Riemensperger et al., 2013). Indeed, Ddc DOPA decarboxylase - is the enzyme that catalyzes the transformation of L-Dopa into dopamine. Taken together these results show that $\mathrm{Hh}$ is necessary to maintain DA homeostasis in adult brain, which is illustrated with the substantial reduction of DA neuron functional integrity observed in $h$ hutant flies.

\section{Adult expression of $\mathrm{Hh}$ is necessary for correct lifespan determination}

We next decided to investigate whether the $h h$ mutant flies exhibit any survival or behavioral deficits, since defects in the dopaminergic neuron network have been demonstrated to have a detrimental effect upon lifespan and locomotor activity in both mammals and invertebrates (Thanos et al., 2016; Liao et al., 2017; Feany and Bender, 2000). Strikingly, we found that adult $h h^{\text {ts }}$ flies shifted to $29^{\circ} \mathrm{C}$ exhibit a drastically shortened lifespan (80 - 85\% decrease in the median lifespan when compared to control flies), both females and males (Fig. 2a, S3c and Table S2). When maintained at $25^{\circ} \mathrm{C}$, but not at $18^{\circ} \mathrm{C}$, the $h h^{\text {ts }}$ mutant flies also exhibited a strongly decreased lifespan (Fig. S3k, Table S2).

To confirm that the loss of $\mathrm{Hh}$ is responsible for this defect, we analysed the heteroallelic combination of $h h^{t s}$ null flies with $h h^{15}$, another strong allele of $h h$. The $h h^{t s} / h h^{15}$ mutant combination reduced lifespan by a similar degree as $h h^{t s}$ (Fig. 2a and Table S2). In addition, $h h^{t s}$ adult mutant flies exhibited diminished climbing ability when compared to age-matched controls (Fig. $2 b$ and Table S3). To further confirm that this effect is specifically due to $h h$ loss of function, re-expressing $h h$ exclusively into glutamatergic neurons in $h h^{t s}$ mutant flies potently rescued the median lifespan (red curve Fig. $2 \mathrm{e}$ ), reaching $68 \%$ of the control one (green curve Fig. $2 \mathrm{e}$ ), whereas expression in GABAergic neurons was sufficient to rescue median lifespan in $h h^{\text {ts }}$ mutant flies up to 41\% (S3a and Table S2). The $h h^{t s}$ climbing defects were also significantly rescued even 
in advanced aged flies (Fig. 2f, S3b and Table S3). The individual UAS and Gal4 transgenes alone had no significant effect on survival rate compared to control flies (Fig. S3d-e and Table S2). These results suggest that Hh expression in the adult fly brain is essential for correct lifespan determination and locomotion activity.

\section{Increased level of Hh results in lifespan extension.}

Then we tested whether increasing $\mathrm{Hh}$ level at adult stage extends lifespan. When $h h$ was overexpressed ( $h h \mathrm{OE}$ ) in an adult specific manner using a driver under the control of the $h h$ promoter (see Methods and Table S2), we observed a considerable expansion $(21 \%)$ of the median lifespan accompanied with a significantly improved climbing ability (Fig. 2a, b and Table S2 and S3). Interestingly, the $h$ h OE-dependent phenotypes were almost completely abolished upon inhibition of neuronal hh OE (using elav-Gal80 repressor of Gal4, see Methods) (Fig. 2c-d, Table S2 and 3). This effect was not observed upon expression of Gal80 in glial cells (using repo-Gal80; Table S2). Altogether, these data suggest that the $h h$-dependent lifespan extension and fitness relates to $h h$ expression in adult neurons, and that potentially a sub-population of both GABAergic and glutamatergic $h h$-expressing neurons contribute to this process.

\section{Hh signalling in glial cells regulates lifespan determination.}

In order to confirm the function of the Hh signaling module in glia, we analysed the consequence of smo or ci depletion in these cells. Glia-specific RNAi for smo or ci (using repo-Gal4) led to a strong reduction in survival rates, with a decrease of $43 \%$ and $48 \%$ in the median lifespan respectively (Fig. 3a and Table S2). In contrast, ci and smo depletion in neurons (using elav-Gal4, Fig. 3b and Table S2) only had a minor effect on survival rates. Furthermore, expressing an activated form of $\mathrm{Ci}$ ( $\mathrm{Ci}^{\text {act. }}$; Wang et al., 1999) in glial cells at 29 or $25^{\circ} \mathrm{C}$ strongly rescued the $h h^{t s}$ mutant shortened median lifespan and defective climbing ability (Fig. 3c-d, S4a-a", S3k and Tables S2 and S3). Additionally, overexpression of $\mathrm{Ci}^{\text {act }}$ or an activated form of Smo (Smo ${ }^{\text {act }}$, Zhang et al, 2004) in glia was sufficient to extend median survival time by $13 \%$ and $16 \%$ respectively (Fig. 3e-f). In all these experiments, the presence of each individual UAS transgene had a negligible effect on survival time (Fig. S3f-j and Table S2). 
To confirm that the DA neuron defects we observed in $h h$ mutant brains (described in Fig. 1) depends on the Hh signalling cascade in glial cells, we analysed whether expressing $\mathrm{Ci}^{\text {act }}$ in glial cells of a $h h$ adult mutant rescues the integrity of DA neurons. Indeed, expression of $\mathrm{Ci}{ }^{\text {act }}$ in glia in a $h$ hutant background strongly recovered the DA neuron volume (Fig. 3g-m) as well as the low neuronal TH mRNA expression and reduced dopamine content (Fig. 3n-o). Overall our data demonstrates that transduction of $\mathrm{Hh}$ signal through Smo and $\mathrm{Ci}$ activity in glial cells is necessary and sufficient for $h h$ dependent lifespan and fitness. It also shows that Ci-dependent activity in the glia is essential in protecting DA neurons during adult life.

\section{Hh signaling regulates proteostasis in glial cells.}

To identify the Hh targets involved in neuroprotection and lifespan determination, we searched for potential protective genes downstream of $\mathrm{Hh}$-dependent transcription in the adult brain. We performed a selective qRT-PCR screen for anti-oxidant defense molecules, autophagy components as well as chaperones (Fig. 4a and S5b-d), demonstrated to be implicated in lifespan regulation (Sun et al., 2012; Simonsen et al., 2008; Tower, 2011). As proof of principle, we examined well-established Hh specific targets regulated at the transcriptional level, such as patched (ptc, the canonical $\mathrm{Hh}$ receptor), decapentaplegic (dpp, of the TGFbeta family) and knot/collier (of the EBF transcription factor family) as well as other members of the Hh module which are posttranslationally regulated (ci and smo; Fig. S5a). In agreement with reports analysing $\mathrm{Hh}$ targets during development (Biehs et al, 2010), we found that ptc was specifically downregulated in brains of flies depleted for adult Hh activity. Out of the protective factors tested in aged $h h$ mutant adult brain samples, a distinct set of three chaperones, the heat shock proteins Hsp23, Hsp40 and Hsp68, displayed a significant mRNA level decrease, when compared to controls (Fig. 4b). Importantly, the expression of $\mathrm{Ci}^{\text {act }}$ in glial cells of $h$ mutants potently rescued the mRNA levels of $h s p 23, h s p 40$ and $h s p 68$ (Fig. 4b), supporting the notion that $h s p$ transcriptional downregulation is a consequence of the loss of Hh signaling. Furthermore, mRNA levels of both $h s p 40$ and $h s p 68$ are similar in agedmatched control flies at both $25^{\circ} \mathrm{C}$ and $29^{\circ} \mathrm{C}$, whereas both chaperone mRNA levels are drastically elevated at $37^{\circ} \mathrm{C}$ heat shock conditions (Fig. 4c-d). This shows that lifespan 
condition run at $29^{\circ} \mathrm{C}$ exhibit no significant difference in regards to the mRNA levels of hsp40 and $h s p 68$ compared to $25^{\circ} \mathrm{C}$ conditions.

Because of the well-accepted role of Hsps in regulating proteostasis (Lindquist and Craig, 1988) we hypothesised that the decrease in $h s p 40$ and $h s p 68$ expression level that we observed in $h$ mutant adult flies may promote accumulation of protein aggregates. We tested this hypothesis using a specific antibody detecting ubiquitin-positive aggregates in the Drosophila brain (Nezis et al., 2008). Accordingly, we observed that old hh mutant flies exhibited a strikingly elevated level of ubiquitinated aggregates when compared to age-matched control flies (Fig. 4e-g, 4l-l'). Moreover, this appeared predominantly localised in midbrain glia tracts, which are enriched for the proteoglycan Perlecan (Pcan; Davie et al., 2018), and also express Ci (Fig. 4g, S5m-p). In addition, ubiquitinated aggregates were also detected in glial cells of the anterior and posterior brain regions of the $h$ h mutant fly brain (Fig. S5e-h, i-I). These observations strongly suggest that a reduction in $h s p 23, h s p 40$ or $h s p 68$ in $h$ h mutant may cause the formation of ubiquitin positive protein aggregates glial cells. Accordingly, when either hsp40 or $h s p 68$ were overexpressed in glial cells of aged adult $h h$ mutants, they strongly reduced ubiquitinated aggregates in glial cells (Fig. 4h-l').

An additional readout for proteostasis function is the measure of pEIF2 $\alpha$ levels which is indicative of the presence of misfolded proteins. The unfolded protein response (UPR) in the Endoplasmic reticulum (ER) activates a signalling cascade which elevates pEIF2 $\alpha$ levels (Halliday et al., 2017). We tested this using the pEIF2 $\alpha$ antibody, which has been used to quantify pEIF2 $\alpha$ in Drosophila (Edenharter et al, 2018). Interestingly we found that $h h$ mutant adult brains exhibited a two-fold increase in pEIF2 $\alpha$ levels, which are strongly reduced to wild type control levels in $h h$ mutant adult flies expressing $h s p 68$ in the glia (Figure 4m-n). Conversely Hh overexpressing flies exhibit a $32 \%$ reduction in pEIF2 $\alpha$ levels compared to age-matched control flies (Fig. S5t-u).

Finally, to ascertain whether $h s p 40$ or $h s p 68$ contribute to $h h$-dependent survival and fitness, we specifically expressed each of them in glial cells of $h h$ mutant flies (Fig. 4o-p and Fig. S4b-d). Remarkably, we found that expression of either hsp68 or hsp40 potently increases the reduced lifespan observed in hh mutant animals (Fig 4o, S4b-c"). In contrast, hsp23 alone was ineffective (Fig. S4d-d"). We also observed that glial 
expression of $h s p 68$ rescued the defective climbing ability of $h h$ mutants by $57 \%$ (Fig. 4p). Overall, our data demonstrates that the $\mathrm{Hh}$ signalling in glial cells promotes expression of protective chaperones required for maintaining correct proteostasis during adult life.

\section{Hh signalling in the glia has a protective function in a human Amyloid beta disease model.}

Defects in glial proteostasis observed upon Hh depletion and their rescue via expression of $\mathrm{Hh}$ target chaperones in the glia, raises the possibility that stimulating $\mathrm{Hh}$ signalling in the glia may reduce aggregate formation and its concommitant toxicity. In Drosophila, expression of the human Amyloid- $\beta 1-42(A \beta 1-42)$ in the glia induces abnormal aggregate formation, reduced lifespan and defective locomotor activity (Jonson et al, 2018). Consistent with this study we expressed, in the adult Drosophila glia, the human arctic variant of $A \beta 1-42\left(A \beta 1-42^{E 693 G}\right)$ which is known to have a high propensity to aggregate and form fibrils in vitro (Nilsberth et al, 2001). We observed the formation of widespread amyloid aggregates throughout the adult brain (Fig. S6a-h.). This phenomenon is also noticeable in the giant glial cells of the optic chiasm (dashed box Fig. S6e and g) in the midbrain sections. The DAPI positive cells exhibit an abnormal morphology with a markedly reduced cell number in this region (Fig. S6g") compared to age-matched wild type control, which exhibit no detectable amyloid aggregates (Fig. S6f, $h-h ")$. Expression of human $A \beta 1-42^{E 693 G}$ in the glia also leads to reduction in lifespan as well as an age-dependent decline in locomotor activity (Fig. 5a-c).

Stimulation of the Hh pathway in glial cells expressing human A $\beta 1-42^{E 693 G}$ via expression of $\mathrm{Smo}^{\text {act }}$ reduced the presence of amyloid aggregates in the giant glial cells of the optic chiasm and rescued DAPI positive cells in this region (Fig. 5g-g"', S6i-i'", Fig 5j-k), and significantly rescued shortened lifespan and locomotion defects (Fig. 5a, c). The rescues were more effective in the $A \beta 1-42^{E 693 G}$ in young animals ( $80 \%$ survival rate) expressing Smo ${ }^{\text {Act }}$ with a rescue of $40 \%$ and a $69 \%$ rescue of debilitated locomotor activity at day 14 (Fig. 5a, c, Table S2-S3). Similarly, the expression of Hsp68 in the glia of A $\beta 1-42^{\text {E693G }}$ expressing animals improved longevity and locomotion by $43 \%$ and $88 \%$, respectively (Fig 5b-c, Table S2-S3) as well as a reduction in the presence of amyloid 
aggregates in the glia and a more potent rescue of cell number (Fig. 5h-h"',5j-k and S6jj"').

Taken together our study suggests that activating $\mathrm{Hh}$ signalling in the glia is protective against abnormal proteostasis. Our proof of concept study utilizing glial expression of the human $A \beta 1-42^{\mathrm{E} 693 \mathrm{G}}$ artic variant, provides an indicator for the protective capacity of $\mathrm{Hh}$ signalling in a disease paradigm.

\section{DISCUSSION}

Our findings provide evidence for a previously unidentified function for the Hh morphogen during adulthood distinct from its role as a patterning organizer and a tumorigenic factor (Briscoe and Thérond, 2013). Our results suggest that Hh signalling in the glia of the adult brain controls the expression of chaperone molecules, including Hsp40 and Hsp68, which repress the formation of age-associated protein aggregates. We propose a model in which $\mathrm{Hh}$ morphogen signaling, interpreted by glial cells, regulates lifespan determination and associated fitness, whilst maintaining adult brain homeostasis, including DA neuron protection (Figure 6).

\section{Hh signaling in glial cells as a lifespan determinant}

There is an emerging body of evidence that diverse glial cell dysfunction has an impact on neuroprotection and organismal lifespan. For instance, disturbance of mitochondrial function by frataxin silencing (Navarro et al., 2010) or impairment of glycolysis via depletion of glycolytic enzymes in the glial cells reduces lifespan and induces neurodegeneration in Drosophila (Volkenhoff et al., 2015; Miller et al., 2016). Additionally activation of the innate immunity response, namely the IMD/NF- $\kappa B$ pathway, in glial cells resulted in an increased expression of antimicrobial peptides, inducing neurodegeneration and diminishing lifespan (Kounatidis et al., 2017). Conversely, glial specific suppression of the IMD/NF- $\kappa B$ signalling pathway culminated in an increased presence of the AKH hormone and lifespan extension (Kounatidis et al., 2017). Furthermore, it has been proposed that the glial neuropeptide RGBA-1 is able to modulate the rate of ageing in C. elegans (Yin et al., 2017). Overall our study reinforces the above 
notion that glia have an important function in the modulation of lifespan and neuroprotection.

Indeed, our study revealed that the Hh signalling pathway is a novel regulator of glial proteostasis. The Hh targets in this cell type, namely Hsp40 and Hsp68, are highly conserved chaperones, (for which the Hsp40 and Hsp70 chaperone family members are the conserved homologues in mammals). These chaperones form part of a major protein complex, which recognises and binds ubiquitinated misfolded proteins, preventing their aggregation and facilitating their refolding or degradation (Lindquist et al., 1988; Jackrel and Shorter, 2017; Zarouchlioti et al., 2018). Interestingly, Hsp40 and Hsp70 act cooperatively as safeguards to prevent both the misfolding of newly synthesized proteins and protein aggregation upon cellular stress. A third Hh target, Hsp23, classified as a small heat shock protein "holdase", binds protein folding intermediates to prevent aggregation, but, in contrast to the Hsp40/70 complex, is unable to refold protein into their native states (Morrow et al., 2015). This functional difference could provide an explanation for the lack of rescue when Hsp23 is expressed in glia of hh mutant animals. Our study demonstrates that glial overexpression of $\mathrm{Hsp40}$ or Hsp68 is able to rescue proteostasis defects present in the $h h$ mutant glial cells, characterized by an elevated level of ubiquitin. Interestingly, $h h$ mutant flies also exhibit increased levels of pEIF2 $\alpha$, indicative of activation of the unfolded proteins response (Halliday et al., 2017), which can be restored to wild type levels through glial expression of Hsp68. Conversely, Hh overexpressing flies display reduced levels of pEIF2 $\alpha$, suggesting that stimulating Hh signalling is protective against protein misfolding.

Some unanswered questions remain, for instance what subtype of glial cells are responsible for mediating $\mathrm{Hh}$ coordinated adult survival and neuronal integrity. One possibility is, that the cortex glia which envelopes neuronal cell bodies could perform this function. It has been suggested that this subtype can provide trophic support to neurons (Stork et al, 2012). Alternatively, the ensheathing glia or the surface glia forming the glia of the blood brain barrier may be the location of Hh mediated neuroprotection. Further investigation is required to address the contribution of each glial subtype to Hh mediated survival and fitness. 
A further line of inquiry to address would be the degree to which protective $\mathrm{Hh}$ signalling in the glia is acting in a cell/non-cell autonomous manner. Given that both, Hsp40 and Hs68, were demonstrated in our study to reduce the presence of ubiquitin positive aggregates in the glia as well as rescue survival rates of $h h$ mutant flies suggests that they act in an autonomous manner, maintaining glial proteostasis. A recent study however demonstrated that both, Hsp40 and Hsp70, can be secreted on exosomes and exert a paracrine neuroprotective effect in an in vitro co-culture experiment (Takeuchi et al., 2015). This suggests that the chaperone targets of Hh signalling may be secreted from the glia and thereby contribute towards Hh-mediated neuroprotection and lifespan determination in a non-cell autonomous manner.

The potential of Hh signaling in alleviating degenerative disease

As proof of principle we tested components and targets of Hh signalling in an in vivo Alzheimer's disease (AD) model in Drosophila expressing the human arctic variant of $A \beta 1-42$ ( $\left.A \beta 1-42^{E 693 G}\right)$ in the glia. In agreement with a recent study (Jonson et al., 2018), here we show that glial expression of the A $1-42^{\text {E693G }}$ variant shortened lifespan, induced debilitated locomotion and increased the presence of amyloid aggregates. Remarkably, we found that induction of Hh signalling in the glia was able to considerably rescue the $A \beta 1-42^{E 693 G}$ - dependent phenotype, both at the molecular and physiological level, providing the first evidence to our knowledge to confirm the efficacy of Hh signalling in an in vivo AD model. We also show that increasing expression of Hsp68 reduces the level of $A \beta 1-42^{E 693 G}$ aggregates in vivo, which is consistent with a study showing that expression of $\mathrm{Hsp} 70$ reduces the level of amyloid $\beta$ aggregates in transgenic mice brains expressing a variant of the amyloid precursor protein (Hoshino et al., 2011). Overall we propose that targets under the regulation of Hh signaling in the glia have the potential to ameliorate proteinopathies, such as those caused by A $\beta$ 1-42 in Alzheimer's disease. It is now proposed that the glia play an important role in various proteinopathies and could function as carriers for the initiation and propagation of aggregates in the adult brain. In fact it has been demonstrated that glial cells can replicate and seed $A \beta$ amyloid fibrils in the mammalian brain independent of neuronal expression (Veeraraghavalu et al., 2014). Thus, activation of the Hh signalling pathway in the glia may have beneficial effects in 
combating a wide-range of proteinopathies which manifest themselves through aberrant protein aggregation and misfolding as observed in Parkinson's, Alzheimer and Huntington diseases.

\section{A conserved role for Hh signaling in healthspan and neuroprotection}

We propose that this new function for Hh signaling as a lifespan determinant may be conserved in mammals. All molecular players described here are conserved; the vertebrate counterpart of Drosophila $\mathrm{Hh}$, Shh, is also expressed in mouse adult neurons and glial cells respond to this signal (Garcia et al., 2010, Farmer et al., 2016). Since the activation of Shh signalling protects against diverse neurotoxins such as amyloid beta peptide in vitro (Yao et al., 2017), our study suggests that Hh signaling in glia may ameliorate age-related neurodegenerative diseases caused by aberrant protein aggregation as well as prolong healthspan and fitness in mammals. 
KEY RESOURCES TABLE

\begin{tabular}{|c|c|c|}
\hline REAGENT or RESOURCE & SOURCE & IDENTIFIER \\
\hline \multicolumn{3}{|l|}{ Antibodies } \\
\hline Oligomer A11 polyclonal antibody & Thermo Fischer & Cat \# AHB0052 \\
\hline Anti-Cubitus Interruptus (Ci) 2A1 & DSHB & Cat \# AB_2109711 \\
\hline Anti-Draper 8A1 & DSHB & Cat AB_2618106 \\
\hline Anti-Elav 7E8A10 & DSHB & Cat \# AB_528218 \\
\hline Anti-Repo 8D12 & DSHB & Cat \# AB_528448 \\
\hline Anti-Smoothened (Smo) $20 \mathrm{C} 6$ & DSHB & Cat \# AB_528472 \\
\hline Anti-GFP antibody & Abcam & Cat \# AB_13970 \\
\hline Anti-Tyrosine Hydroxylase (TH) & Millipore & Cat \# AB_152 \\
\hline Anti-Ubiquitin (Ubq) FK2 & Enzo Biosciences & Cat \# BML-PW8805 \\
\hline Anti-Perlecan & Laboratory & N/A \\
\hline Anti-phospho elF2a & Abcam & Cat \# AB_32157 \\
\hline \multicolumn{3}{|c|}{ Chemicals, Peptides, and Recombinant Proteins } \\
\hline Amyloid Tracker 680 & Ebba Biotech & Cat \# AmyTracker ${ }^{\mathrm{Tm}} 680$ \\
\hline $\begin{array}{l}\text { Vectashield Fluorescent Mounting } \\
\text { Medium }\end{array}$ & Vecta Laboratories & Cat \# H-1200 \\
\hline \multicolumn{3}{|l|}{ Critical Commercial Assays } \\
\hline Bio-Rad DC Protein Assay Kit II & BioRad & Cat\# 500-0112 \\
\hline \multicolumn{3}{|l|}{ Experimental Models: Organisms/Strains } \\
\hline D. Melanogaster: Hh-Gal4 & $\begin{array}{l}\text { Bloomington } \\
\text { Drosophila Stock } \\
\text { Centre (BDSC) }\end{array}$ & $\begin{array}{l}\text { BDSC: } 67046 \\
\text { Fly Base: FBti0017278 }\end{array}$ \\
\hline $\begin{array}{l}\text { D. Melanogaster: Hh-Gal4 T2A } \\
\text { (Trojan) }\end{array}$ & $\begin{array}{l}\text { Bloomington } \\
\text { Drosophila Stock } \\
\text { Centre (BDSC) }\end{array}$ & $\begin{array}{l}\text { BDSC: } 67493 \\
\text { Fly Base: FBti0186278 }\end{array}$ \\
\hline $\begin{array}{l}\text { D. Melanogaster: Ci-Gal4 T2A } \\
\text { (Trojan) }\end{array}$ & $\begin{array}{l}\text { Laboratory of Stephan } \\
\text { Schneuwly, } \\
\text { Regensburg } \\
\text { University }\end{array}$ & N/A \\
\hline D. Melanogaster: C155-elav-Gal4 & $\begin{array}{l}\text { Bloomington } \\
\text { Drosophila Stock } \\
\text { Centre (BDSC) }\end{array}$ & $\begin{array}{l}\text { BDSC: 458; } \\
\text { Fly Base: FBti0002575 }\end{array}$ \\
\hline D. Melanogaster: repo-Gal4 & $\begin{array}{l}\text { Bloomington } \\
\text { Drosophila Stock } \\
\text { Centre (BDSC) }\end{array}$ & $\begin{array}{l}\text { BDSC: } 7415 \\
\text { Fly Base: FBti0018692 }\end{array}$ \\
\hline
\end{tabular}




\begin{tabular}{|c|c|c|}
\hline D. Melanogaster: TH-Gal4 & $\begin{array}{l}\text { Bloomington } \\
\text { Drosophila Stock } \\
\text { Centre (BDSC) }\end{array}$ & $\begin{array}{l}\text { BDSC: } 8848 \\
\text { Fly Base FBtp0114847 }\end{array}$ \\
\hline D. Melanogaster: ChAT-Gal4 & $\begin{array}{l}\text { Bloomington } \\
\text { Drosophila Stock } \\
\text { Centre (BDSC) }\end{array}$ & $\begin{array}{l}\text { BDSC: } 6798 \\
\text { Fly Base FBst0006798 }\end{array}$ \\
\hline D. Melanogaster: ddc-Gal4 & $\begin{array}{l}\text { Bloomington Drosophila } \\
\text { Stock Centre (BDSC) }\end{array}$ & $\begin{array}{l}\text { BDSC: } 7010 \\
\text { Fly Base:FBst0007010 }\end{array}$ \\
\hline D. Melanogaster: vGlut-Gal4 & $\begin{array}{l}\text { Bloomington Drosophila } \\
\text { Stock Centre (BDSC) }\end{array}$ & $\begin{array}{l}\text { BDSC: } 26160 \\
\text { Fly Base FBti0076967 }\end{array}$ \\
\hline D. Melanogaster: Gad-Gal4 & $\begin{array}{l}\text { Bloomington Drosophila } \\
\text { Stock Centre (BDSC) }\end{array}$ & $\begin{array}{l}\text { BDSC: } 51630 \\
\text { Fly Base FBtp0016769 }\end{array}$ \\
\hline D. Melanogaster: Tdc2-Gal4 & $\begin{array}{l}\text { Bloomington Drosophila } \\
\text { Stock Centre (BDSC) }\end{array}$ & $\begin{array}{l}\text { BDSC: } 9313 \\
\text { Fly Base FBti0101786 }\end{array}$ \\
\hline D. Melanogaster: w1118 & $\begin{array}{l}\text { Bloomington Drosophila } \\
\text { Stock Centre (BDSC) }\end{array}$ & $\begin{array}{l}\text { BDSC: } 5905 \\
\text { Fly Base FBst0005905 }\end{array}$ \\
\hline D. Melanogaster: elav-Gal80 & $\begin{array}{l}\text { Laboratory of Sean } \\
\text { Sweeney, University } \\
\text { of York }\end{array}$ & N/A \\
\hline D. Melanogaster: repo-Gal80 & $\begin{array}{l}\text { Laboratory of Tzumin } \\
\text { Lee, Univesity of } \\
\text { Massachusetts }\end{array}$ & N/A \\
\hline D. Melanogaster: Gal80 Ts & $\begin{array}{l}\text { Bloomington } \\
\text { Drosophila Stock } \\
\text { Centre (BDSC) }\end{array}$ & $\begin{array}{l}\text { BDSC: } 7017 \\
\text { Fly Base: FBst0007017 }\end{array}$ \\
\hline D. Melanogaster: hh-dsRed & $\begin{array}{l}\text { Laboratory of Shigeo } \\
\text { Hayashi, Kobe } \\
\text { University }\end{array}$ & N/A \\
\hline D. Melanogaster: UAS: GFP NLS & $\begin{array}{l}\text { Bloomington } \\
\text { Drosophila Stock } \\
\text { Centre (BDSC) }\end{array}$ & $\begin{array}{l}\text { BDSC: } 4776 \\
\text { Fly Base: FBst0004776 }\end{array}$ \\
\hline D. Melanogaster: UAS:mCD8 GFP & $\begin{array}{l}\text { Bloomington } \\
\text { Drosophila Stock } \\
\text { Centre (BDSC) }\end{array}$ & $\begin{array}{l}\text { BDSC: } 5137 \\
\text { Fly Base: FBst0005137 }\end{array}$ \\
\hline $\begin{array}{l}\text { D. Melanogaster: } 6 \text { x UAS:mCD8 } \\
\text { mCherry }\end{array}$ & $\begin{array}{l}\text { Bloomington } \\
\text { Drosophila Stock } \\
\text { Centre (BDSC) }\end{array}$ & $\begin{array}{l}\text { BDSC: } 52268 \\
\text { Fly Base: FBti0162761 }\end{array}$ \\
\hline D. Melanogaster: UAS:Dcr2 (2) & $\begin{array}{l}\text { Bloomington } \\
\text { Drosophila Stock } \\
\text { Centre (BDSC) }\end{array}$ & $\begin{array}{l}\text { BDSC: } 24650 \\
\text { Fly Base: FBst0024650 }\end{array}$ \\
\hline D. Melanogaster: UAS:Dcr2 (3) & $\begin{array}{l}\text { Bloomington } \\
\text { Drosophila Stock } \\
\text { Centre (BDSC) }\end{array}$ & $\begin{array}{l}\text { BDSC: } 24651 \\
\text { Fly Base: FBti0100276 }\end{array}$ \\
\hline D. Melanogaster: UAS:Ci ${ }^{\text {Act }}$ & $\begin{array}{l}\text { Laboratory of Konrad } \\
\text { Basler, University of } \\
\text { Zurich }\end{array}$ & N/A \\
\hline D. Melanogaster: UAS:Smo Act & $\begin{array}{l}\text { Laboratory of Phillip } \\
\text { Beachy, Stanford } \\
\text { University }\end{array}$ & $\mathrm{N} / \mathrm{A}$ \\
\hline
\end{tabular}




\begin{tabular}{|c|c|c|}
\hline D. Melanogaster: UAS:hh M4 & $\begin{array}{l}\text { Laboratory of Phillip } \\
\text { Ingham, ICRF, } \\
\text { London }\end{array}$ & N.A \\
\hline D. Melanogaster: UAS:hsp23 & $\begin{array}{l}\text { Bloomington } \\
\text { Drosophila Stock } \\
\text { Centre (BDSC) }\end{array}$ & $\begin{array}{l}\text { BDSC: } 30541 \\
\text { Fly Base: FBti0129804 }\end{array}$ \\
\hline D. Melanogaster: UAS:hsp40 & $\begin{array}{l}\text { Bloomington } \\
\text { Drosophila Stock } \\
\text { Centre (BDSC) }\end{array}$ & $\begin{array}{l}\text { BDSC: } 30553 \\
\text { Fly Base: FBst0030553 }\end{array}$ \\
\hline D. Melanogaster: UAS:hsp68 & $\begin{array}{l}\text { Laboratory of Heinrich } \\
\text { Japer, Buck Institute } \\
\text { for Research on } \\
\text { Ageing }\end{array}$ & N/A \\
\hline $\begin{array}{l}\text { D. Melanogaster: UAS: Aß42 1- } \\
\text { 42. } \text { E693G }^{-}\end{array}$ & $\begin{array}{l}\text { Bloomington } \\
\text { Drosophila Stock } \\
\text { Centre (BDSC) }\end{array}$ & $\begin{array}{l}\text { BDSC: } 33773 \\
\text { Fly Base: FBti0141194 }\end{array}$ \\
\hline D. Melanogaster: UAS: Ci RNAi & $\begin{array}{l}\text { National Institute of } \\
\text { Genetics (NIG) Japan }\end{array}$ & 2152R-1 \\
\hline D. Melanogaster: UAS: Smo RNAi & $\begin{array}{l}\text { National Institute of } \\
\text { Genetics (NIG) Japan }\end{array}$ & 11561R-2 \\
\hline D. Melanogaster: Ci-LexA & $\begin{array}{l}\text { Bloomington } \\
\text { Drosophila Stock } \\
\text { Centre (BDSC) }\end{array}$ & $\begin{array}{l}\text { BDSC: } 54211 \\
\text { Fly Base: } \\
\text { FBsf0000163705 }\end{array}$ \\
\hline $\begin{array}{l}\text { D. Melanogaster: } 16 \text { x LexAop2- } \\
\text { mCD8 ::GFP }\end{array}$ & $\begin{array}{l}\text { Bloomington } \\
\text { Drosophila Stock } \\
\text { Centre (BDSC) }\end{array}$ & $\begin{array}{l}\text { BDSC: } 32206 \\
\text { Fly Base: FBti0131945 }\end{array}$ \\
\hline D. Melanogaster: $\mathrm{hh}^{\mathrm{ts} 2}$ & $\begin{array}{l}\text { Bloomington } \\
\text { Drosophila Stock } \\
\text { Centre (BDSC) }\end{array}$ & $\begin{array}{l}\text { BDSC: } 1684 \\
\text { Fly Base: FBst0001684 }\end{array}$ \\
\hline D. Melanogaster: $\mathrm{hh}^{15}$ & $\begin{array}{l}\text { Laboratory of Jym } \\
\text { Mohler, Columbia } \\
\text { University }\end{array}$ & N/A \\
\hline D. Melanogaster: $\mathrm{Smo}^{\mathrm{X} 43}$ & $\begin{array}{l}\text { Laboratory of Marek } \\
\text { Mlodzik, University of } \\
\text { Heidelberg }\end{array}$ & N/A \\
\hline
\end{tabular}

Software and Algorithms

\begin{tabular}{lll}
\hline Biorad CFX manager 3.1 Software & GraphPad & $\begin{array}{l}\text { http://www,graphpad.com/sci } \\
\text { entific-software/prism/ }\end{array}$ \\
\hline Prism Software &
\end{tabular}

\section{EXPERIMENTAL PROCEDURES}

\section{Fly Stocks and Lifespan/Locomotion Assays}

Flies were raised on standard media at $18^{\circ} \mathrm{C}$ for all experiments and transferred to $29^{\circ} \mathrm{C}$, no longer than 24 hours after adult eclosion, to ensure adult specific inactivation of the $h h$ mutants and adult specific expression of the relevant transgenes of the Hh signalling 
pathway using the Gal80 ${ }^{\text {ts }}$ UAS/GAL4 system during adulthood as well as to avoid developmental defects prior to adult eclosion. Stock lines and GAL4 driver lines were obtained from the Drosophila Stock center at Bloomington, or are described in the methods text. Flies were generated or backcrossed a minimum of 5 generations into a controlled uniform homogeneous genetic background (Flybase ID FBst0005905, w1118), in order to ensure that all phenotypes were robust and not associated with variation in genetic background. In this uniform homogeneous genetic background, the survival time of control flies is highly reproducible. Startled-induced negative geotaxis was used to assess fly locomotion. To determine lifespan, newly eclosed females were mated for 24 to 48 hours at $18^{\circ} \mathrm{C}$ collected and maintained at 20 flies per vial and transferred to fresh 11 vials every two days while being scored for survival. A minimum of 180 to 300 flies were used per genotype per lifespan assay, unless otherwise indicated.

\section{Immunohistochemistry}

Adult male heads were dissected and immunostained with the specified antibodies using standard Procedures (Wu and Luo, 2006).

\section{Statistical Analysis}

Survival assays were analyzed in Excel (Microsoft) and in Prism software (GraphPad) for survival curves and statistics.

\section{qRT-PCR analysis and Western Blots}

Techniques of molecular biology, and histology were standard. Expression of genes from fly heads extracts was carried out using standard procedures for mRNA extraction, cDNA synthesis and semi-quantitative Real Time PCR.

\section{Supplementary Information}

Supplemental information includes Supplemental Experimental Procedures, six figures and six tables can be found with this article: 


\section{Supplemental Experimental Procedures}

\section{Genetic background}

All flies were generated in the same uniform homogeneous genetic background in order to prevent genetic background effects, (line 5905 (Flybase ID FBst0005905, w1118)), and backcrossed a minimum of 5 generations into this uniform w1118 genetic background. This ensures that the observed phenotypes were due to gene manipulations, and not variation in genetic background. In these carefully controlled experiments, the lifespan of control flies was highly uniform and repeatable when 180 or more individuals were used for lifespan analysis (see Table S1). The short hand and the corresponding full-genotypes as well as explanation of the components utilised for the lifespan/mobility experiments are listed in Table S4.

\section{Fly stocks and genetics}

The UAS/GAL4/GAL80 ${ }^{\text {ts }}$ system was utilised (Brand and Perrimon., 1993) to drive adult tissue-specific protein expression of the relevant UAS: transgenic lines and to avoid developmental defects prior to adult eclosion. Gal80 ${ }^{\text {ts }}$ is an effective repressor of Gal4 activity at $18^{\circ} \mathrm{C}$. At $25^{\circ} \mathrm{C}$ or $29^{\circ} \mathrm{C} \mathrm{Gal} 80^{\text {ts }}$ is partially or completely inhibited, allowing Gal 4 activity thereby driving the expression of the relevant UAS transgene. The following Gal4/Gal80 strains were used in this study: hh-Gal4, elav-Gal4, repo-Gal4, TH-GAL4, ChAT-GAL4, Ddc-Gal4, vGlut-GAL4, Gad-GAL4, Tdc2-GAL4, w1118, elav-Gal80, Gal80 ${ }^{\text {ts }}$ (Bloomington, Drosophila Stock Centre) and repo-Gal80 (a gift from T. Lee, Awakaki and Lee, 2011) hh-dsRed (a gift from S. Hayashi, Akimoto et al, 2005). The following UAS transgenic lines were used: UAS:GFP NLS, UAS:mCD8::GFP, UAS: $h$ h M4, UAS: cict $^{\text {act }}$ UAS: smo Glu ${ }^{(A c t)}$ (Zhang et al, 2004), UAS: Dcr2, UAS: hsp23, UAS: hsp40 (Bloomington Drosophila Stock Centre) and UAS: Hsp68 (a gift from H. Jasper; Wang et al, 2005) The $h h$ mutant allele strains used were as follows: $h h^{t s 2}$, - a temperature sensitive allele shown to be null when inactivated at the non-permissive temperature $29^{\circ} \mathrm{C}$, (Stringini et al, 1997; Palm et al, 2013; Ranieri et al, 2012) and $h h^{15}$, is a presumed null allele (Mohler et al, 1988). The RNAi lines used in this study were: $\mathrm{Ci}$ RNAi (2125R-1), Smo RNAi (11561R-2), from fly stocks of national institute of genetics 
(NIG). The following lexA/LexOp line were used in this study ci-lexA, 16XLexAop2mCD8::GFP (Bloomington Drosophila stock Centre). The $s m o^{I I X 43}$ mutant was a gift from Marek Mlodzik (Nüsslein-Volhard et al, 1994).

\section{Adult specific inactivation of the Hh mutants}

To assess the role of Hh signalling in lifespan determination, we initially examined the temperature sensitive $h h$ mutant $\left(h h^{\text {ts2 }}\right.$ ) strain, which can be considered a $h h$ null (Stringini et al, 1997; Palm et al, 2013; Ranieri et al, 2012) when placed at non-permissive temperature $29^{\circ} \mathrm{C}$ for a minimum of $24 \mathrm{~h}$. We raised the $h h^{\text {ts2 }}$ flies at a permissive temperature of $18^{\circ} \mathrm{C}$ to ensure that eclosed adult flies exhibited no development defects and were viable.

\section{Lifespan assay}

Fly stocks were grown under standard conditions. For adult lifespan assays, parental fly lines were mated at $18^{\circ} \mathrm{C}$ for 48 hours with 20 females and 40 males in $177 \mathrm{ml}$ polypropylene bottles containing $50 \mathrm{ml}$ of standard $1 \mathrm{x}$ yeast food. Males were then removed and the remaining females were allowed to lay embryos within a 24-hour time frame. Female adult progeny were collected at $18^{\circ} \mathrm{C}$ and divided into cohorts of 20 per vial (unless otherwise specified) and mated with control $w 1118$ control males in a 1:3 ratio for a period of a maximum of 48 hours. Males were subsequently removed and cohorts of 20 mated females per vial were transferred to an $29^{\circ} \mathrm{C}$ incubator, with the mortality being measured approximately at the same time of day every 2 days.

\section{Adult locomotion ability}

Startled-induced negative geotaxis was used to assess fly locomotion. To perform this negative geotaxis assay, groups of 10 adult female flies of indicated age were transferred into a polystyrene round-bottom vials $(25 \mathrm{~mm} \times 95 \mathrm{~mm})$ and left to recover at room temperature for 5 minutes. Climbing ability was scored as the percentage of flies failing to climb higher than $5 \mathrm{~cm}$, unless otherwise stated (Table S3) from the bottom of the tube, within 10 seconds after the base of the tube was gently hit. Three repeats were carried 
out for each set of 10 flies and the results averaged. Between each experiment within the same set, flies were given 1 minute to recover prior to the next negative geotaxis experiment and subsequent measurement of climbing ability. For each genotype at a given age, a minimum of 100 flies were examined.

\section{Generation of ci-T2A-GAL4 line}

In order to overcome the lack of reliable Gal4 lines for ci, we generated a new stock using the Trojan Gal4 expression module (T2A-Gal4 cassette). In this approach, the native gene product is cleaved and the translation of GAL4 occurs as an independent protein (Diao et al, 2015). The Trojan module was integrated into the third exon of the ci locus. Two constructs were generated to integrate the construct into the fly genome. Firstly, a construct to express the guide RNA (gRNA) for the targeted cleavage of the DNA at the insertion site, and secondly the construct containing the Trojan cassette flanked by homology arms necessary for integration through homology directed repair. Primers to generate the gRNA (CigRNApU6fw: 5'-CTTCGCATTCCATCTTGCCGGACT-3' and CigRNApU6rv: 5'-AAACAGTCCGGCAAGATGGAATGC-3') were annealed using T4 polynucleotide kinase and cloned into Bbsl-digested pU6-Bbsl-chiRNA vector (Addgene plasmid 45946) as described in www.crisprflydesign.org. The two homology arms were amplified from genomic DNA using the following primers (Hom1ciAgelfw: 5'GGCCACCGGTCATGACCAGTGCAACGCC-3' and Hom1ciSacllrv: 5'GGCCCCGCGGCCCTCGCCGGCAAGATGGAATGCTG-3', hom2ciKpnlfw: 5'GCGCGGTACCACTTGGTTTGGGATCAGCAGCTGATTT-3' and hom2ciSpelrv: 5'GCGCACTAGTCTCAATGCGACAGCAGCTACGCC-3') and cloned into the empty backbone of the pTGEM(0) vector (Addgene plasmid 62891) using Agel and Sacll or Kpnl and Spel, respectively. A solution containing $100 \mathrm{ng} / \mu \mathrm{l}$ of the gRNA in pU6 and $500 \mathrm{ng} / \mu \mathrm{l}$ of pT-GEM(0) was injected into the vas-Cas9 line (number 51324 from Bloomington Stock Center). Positive transformants were identified by the presence of the 3XP3-dsRed module also contained in the pT-GEM vector. The vas-Cas 9 chromosome marked with 3xP3-GFP were excluded during the generation of the final stock. 


\section{Molecular Biology}

\section{Real-Time PCR}

Total RNA was extracted from 50 heads using peqGold TriFast reagent (PEQLAB Biotechnologie GMBH, Erlangen, Germany) following the manufacturer's instructions. $500 \mathrm{ng}$ of mRNA were converted to cDNA using QuantiTect Rev. Transcription Kit (Qiagen $\mathrm{GmbH}$, Hilden, Germany) then used for qPCR with ORA qPCR Green ROX L Mix (HighQu, Kralchtal, Germany) on a CFX connect Real- Time PCR Detection System (Bio-rad, Hercules, California, USA). The ribosomal protein 49 (rp49) was used as internal control. The results from at least four independent biological replicates were analysed with the Bio-Rad CFX manager 3.1 software. Gene expression levels were referred to the internal control, the relative quantification was carried out by means of the $\Delta \Delta \mathrm{Ct}$ method and the results were expressed as relative mRNA expression. Each experiment consisted of 4-5 independent biological replicates. The genes and the pairs of primers used for the analysis are listed below.

Pairs of primers used for qRT PCR analysis.

Gene Forward primer (5'-3')

atg1 (CG10967) GTCAGCCTGGTCATGGAGTAT

atg8a (CG32672) ATCCAGACCGTGTGCCCGTCAT

BiP (CG4147) TGTCACCGATCTGGTTCTTCAGGC

catalase (cat, GGACCAGGGCATCAAGAATCT

CG6871)

hedgehog $(\boldsymbol{h} \boldsymbol{h}$, GGATTCGATTGGGTCTCCTAC

CG4637)

patched (ptc, GGATCTTTACATACGCACCAGC

CG2411)

smoothened (smo, GARGTTCCTGGAGGTTGCGAT

CG11561)

cubitus interruptus GATTTTCGCCAAACTCTTTAGCC
Reverse primer (5'-3')

CGTCCCCTTGACACTCAGAT

ACCGACGGTCAGGTCGGAAGG

GTCCCATGACCAAGGACAACCATC

CTGCTCCACCTCAAGCAAAG

GGGAACTGATCGACGAATCT

GGACTGGAATACTGATCGCAG

AGTCGGTAATTGAGAGTCCCATT

ACATGGGATTAAGGGCGGTAG

(Ci, CG2125) 
decapentaplegic GCCAACACAGTGCGAAGTTT

(dpp, CG9885)

gstD1(CG10045)

hsp22 (CG4460)

hsp23 (CG4463)

hsp26(CG4183)

hsp27(CG4466)

hsp40(CG10578)

hsp67Ba(CG4167)

hsp68(CG5436)

hsc70-4(CG4264)

hsp70Aa(CG31366)

hsp83(CG1242)

knot (Kn, AGCGGACGGCTTTCATTGG

CG10197)

lamp1(CG3305)

nTH (CG10118)

GCTTTCCTTTATGCAAATTCATC

rp49 (CG7939) CCCAGCACTTCATCCGCCACC

sod1(CG11793) TGGACACGAGCTGAGCAAG

sod2(CG8905) AGTCGCAGTGGAAGAGCC
CGGGAATGCTCTTCACGTCG

CAGGTTGAGCAGCTTCTTGTT

CACATTGCTGTATCCCTGACTTTTGG GGCGCCCACCTGTTTCTC CCCTCGACCAAGATGGAGT

CGCGACGTGACATTTGAT

CGGGAAACTTAATGTCGAAGGAGAC CGAGGCAACGGGCACTTC

GTCAACCTTGGGAATACGAGTG TCGTTTGACCCGTTTGTAT CCTGCTTCACATTGAAGACGTA GAAAAACCCGACCCGAACTGGA GCTCCATTTGCGTAGAGTAACTG

GCTGAACCGTTTGATTTTCC CTTGGTCGGCTGTTGGTT GCGGGTGCGCTTGTTCGATCC GTGGCCGACATCGGAATAG AGATGTCATCCCAGTTGG

Immunohistochemisty

Fly brains were dissected at various time points during adulthood as specified and stained as previously described (Wu and Luo, 2006). For visualisation of dopaminergic neurons in the adult brain, the following antibody was used: rabbit anti-TH (millipore). For labelling of the neuron and glia nuclei, mouse anti-Elav: 1:50 (7E8A10 DSHB) and mouse antiRepo: 1:50 (8D12 DSHB) were used respectively. For labelling of the glial cytosol/membrane, mouse anti-Draper: 1:50 (8A1 DSHB) was used (Purice et al, 2016) For labelling glia, rabbit anti-Perlecan 1:100 was used (gift by S.Pizette). Additional antibodies were also used: chicken anti-GFP 1:1000 (Abcam). For examining localisation of $\mathrm{Hh}$ signalling components in the adult brain, the following antibodies were used: mouse 
anti-Smo 1:50 (20C6 DSHB) and rat anti-Ci155 1:10 (2A1 gift from R. Holmgren). For labelling of aggregates in the adult brain: mouse anti-Ubq 1:500 (Enzo Biosciences) was used and for amyloid aggregates, rabbit anti-A11 (Themo Fischer) was utilised. Secondary antibodies used were as follows: goat anti-chicken Alexa488 (1:500; Invitrogen A11039); donkey anti-mouse Alexa488 (1:500; Invitrogen A21202). donkey anti-mouse Alexa546 (1:500; Invitrogen A10036). goat anti-rabbit Alexa546 (1:500; Invitrogen A11035), goat anti-rat Alexa546 (1:500; Invitrogen A11081), goat anti-mouse 647 (1:200: Invitrogen A21236) and adult brains were mounted in Vectasheld (Vecta Laboratories). Samples were embedded in Vectashield IN mounting medium (Vector Laboratories, Burlingame, CA, USA).

In each experiment, at least 4-12 flies were scanned per genotype using the Leica TSC SP5 and SP8 Confocal Laser Scanning Platforms (Leica, Germany). The detector gain and laser settings calibrated at the same level for each experiment.

\section{Western Blot}

For Western blots, 15 adult bodies or 40 to 50 heads were homogenized in $80 \mathrm{ml}$ cold RIPA buffer (Sigma-Aldrich, St. Louis, MO, USA) with complete protease and phosphatase inhibitor mixture (Roche, Mannheim, Germany). The protein levels were determined using the BCA protein quantification kit (Thermo Scientific, Schwerte, Germany). Samples were diluted 1:4 with standard 4_Laemmli buffer, boiled for 5 min and approximately $25-30 \mu$ g of proteins were separated on $5 \%$ stacking-10\% separating SDS polyacrylamide gels. The resolved proteins were electroblotted to a Protran BA 85 nitrocellulose membrane (Hartenstein, Würzburg, Germany) and were probed using rabbit anti-phospho elF2aS1 (1:1500, ab32157, abcam, Cambridge, UK). Mouse anti-atubulin (1:5000, T9026, Sigma-Aldrich, St. Louis, MO, USA) was used as loading controls. Secondary fluorescent goat anti-rabbit 680RD and goat anti-mouse 800CW (1:10000, LiCor Inc., Bad Homburg, Germany) were used in all cases. Detection and quantification was conducted using the Odyssey system (Li-Cor Inc., Lincoln, NE, USA). Arbitrary fluorescent units were normalized to the internal loading control. Data were expressed as percentage of average control values and plotted as relative protein levels. 


\section{DA neuron volume quantification in the adult brain with Imaris software} Dopaminergic neurons were recognised through specific labelling with either mCD8::GFP or the anti-TH immunostaining. We used the IMARIS 8.4.1 module (Bitplane Inc) to process the raw files, which consisted of the entire $3 \mathrm{D} \mathrm{Z}$ stack of the adult brain. The IMARIS 'add new surfaces' option was selected for the relevant channel and the threshold was adjusted until all mCD8::GFP tagged neurons or neurons positive for anti-TH immunostaining were labelled as a discrete surface. Neurons were clearly distinguished from background due to the contiguous nature of their axons. We then measured the volume of DA neurons in each adult brain, using the 'statistics' option. All subsequent DA neuron volume data for each adult brain sample were subsequently exported to Excel. The collated data were further analysed within Prism software (GraphPad) to statistically compare $h h$ mutant and age-matched control samples.

The DA neuron volume network was analysed by labelling their cell membrane with the mCD8-GFP reporter transgene using the Ddc-GAL4 driver in tandem with staining for $\mathrm{TH}$. The $h$ mutant and control samples containing the Ddc-Gal4 driver exhibited extended survival times as a result we quantified DA neuron volume at different time points, young ( 0 to 7 days), middle-aged (day 21) and more aged time points (day 35). The decrease in $\mathrm{TH}$ immunostaining levels characteristic of older $h h$ mutant flies was more delayed and manifested at latter time points. The above variation in lifespan due to the presence of the Ddc-Gal4 driver, is consistent with studies demonstrating that $D d c$ has been implicated as a lifespan determinant (De Luca,et al, 2003; Paaby and Schmidt; 2009)

\section{DA neuron cell counting}

PAM DA neuron clusters were counted with the Fiji cell counting tool in a Z-projection covering the region were PAM DA neuron cell bodies were present.

\section{Dopamine quantifications}

Dopamine levels in Drosophila brain extracts were determined by HPLC coupled to electrochemical detection essentially as described (Hardie and Hirsch, 2006) using a 
mobile phase containing $4 \mathrm{mM}$ decanesulfonic acid, $50 \mathrm{mM}$ citrate/acetate, $\mathrm{pH} 4.5$ and 20\% acetonitrile. The HPLC system consisted of a Jasco model PU-2089 Plus isocratic pump (Jasco Inc., Easton, MD, USA) coupled to an ED703 Pulse electrochemical detector (GL Sciences, Shinjuku-ku, Tokyo, Japan) equipped with a low-volume flow cell. Separation was performed with a $10 \mathrm{~cm}$ x $4.6 \mathrm{~mm}$ Hypersil H3ODS-100A column (Hichrom, Berkshire, UK) protected by a Hypersil guard cartridge. The mobile phase was delivered at $0.3 \mathrm{ml} / \mathrm{min}$ flow rate. The brains of 10 flies per genotype were dissected and directly placed into $50 \mu \mathrm{l}$ of ice-cold $50 \mathrm{mM}$ citrate/acetate, $\mathrm{pH} 4.5$. They were quickly homogenized with a Teflon Pestle (Dominique Dutscher, Brumath, France). The homogenates were run through a nylon $0.2 \mu \mathrm{m}$ centrifugal filter (VWR, Radnor, PA, USA) before analysis. Dopamine detection was performed at a detector potential of $+1,000 \mathrm{mv}$ relative to a conductive diamond electrode. The injection volume was $10 \mu$ l. Three independent determinations were done for each genotype and three injections were done for each determination. Results are presented as mean \pm SEM.

\section{Ubiquitin signal intensity quantifications}

Quantifications of Ubq signal intensity were acquired utilising Image J>Measurement function, from two different regions of the adult fly brain, labelled 1 CB (Central Brain)/OL (Optical Lobe) boundary region and 2 Midline of the Central Brain (CB). The demarcated ROIs are indicated in Figures 4i and S5r, and the equivalent ROI were used for Ubq signal intensity quantifications in all samples analysed (Figure $4 \mathrm{~h}-\mathrm{k}$ and S5q-r). The $1000 \mu \mathrm{m}^{2}$ $(31.62 \mu \mathrm{m} \times 31.62 \mu \mathrm{m}) \mathrm{ROI}$ areas quantified represent the average pixel number in this region per $\mu \mathrm{m}^{2}$ and for all genotypes quantified 12 brain hemispheres were analysed and an average value was acquired.

\section{A11 amyloid aggregate surface area quantifications}

Quantifications of A11 positive amyloid aggregate surface area were acquired utilising Image $\mathrm{J}$ (Figure 5j). The threshold function was utilized to first delineate the presence of amyloid aggregates in the specified ROls and the same threshold intensity of 107 was used to quantify all samples. The demarcated ROls are indicated in Figures $5 \mathrm{~d}-\mathrm{e}$ and S6e-f and i-j, and the equivalent ROI of $43.14 \mu \mathrm{m} \times 91.96 \mu \mathrm{m}$ were used for A11 amyloid 
aggregate surface area quantifications in all samples analysed, within a $5 \mu \mathrm{m}$ Z-projection stack. For all genotypes quantified a minimum of 12 brain hemispheres were analysed within the selected $\mathrm{ROI}$ region $(43.14 \mu \mathrm{m} \times 91.96 \mu \mathrm{m})$, surrounding the giant glial cells of the optic chiasm.

\section{DAPI positive Cell counting}

DAPI positively stained cells in the entire ROI $(43.14 \mu \mathrm{m} \times 91.96 \mu \mathrm{m})$, were counted with the Fiji cell counting tool in a $5 \mu \mathrm{m}$ Z-projection stack for all samples (Figure 5f",g",h" and I", quantifications Figure 5k). A total of 12 samples, from 12 individual adult brains were quantified for each genotype.

\section{Statistical analysis}

Statistical analysis was carried out using prism version 7.02 for windows, graphpad software, (La Jolla, California USA, www.graphpad.com). For lifespan analysis the log rank test was utilized, to compare median lifespans of two specified data sets. In the case of survivals assays (Figure $3 \mathrm{~b}$ ) where error bars (SEM) overlapped between two data sets (Figure 3b) cox-regression (cr) analysis was used to calculate statistical significance in these instances. For climbing assays and DA neuron volume quantification, two ways analysis of variance (ANOVA) was used to compare the specified data points ${ }^{* * *}, p<$ $0.001 ;{ }^{* *}, p<0.01$ and $\left.{ }^{*}, p<0.05\right)$, whereas for quantification of DA neuron number, Ubq signal intensity, and A11 positive amyloid aggregates, the two-tailed t-test was used.

Analysis of Real Time PCR were performed as follows: i) when comparing 2 samples, equal variances were confirmed by an $\mathrm{F}$ test. ii) when comparing multiple samples, equal variances were confirmed by Bartlett and Brown-Forsythe tests. In all cases normality of data was assessed with Shapiro \& Wilk test and parametric or non-parametric tests were used accordingly. Since all data passed normality test, significance was determined by two-tailed T-test or by One-way ANOVA with post hoc Tukey Multiple Comparison Test ${ }^{* * *}, \mathrm{P}<0.001 ;{ }^{* *}, \mathrm{P}<0.01$ and $\left.{ }^{*}, \mathrm{P}<0.05\right)$. Statistical analysis was carried out using Prism version 7.02 for Windows, GraphPad Software, La Jolla California USA, www.graphpad.com 


\section{Figure legends}

Figure 1. Location of Hh signalling components in the Drosophila adult brain and DA neuron loss in $h h$ mutants

(a) Schematic representation of the Ci-T2A-Gal4 trojan line. (b-b") Cells positive for ci T2A-Gal4 (trojan line) expressing GFP NLS (green channel - b) are immunostained with the glial marker Repo (red channel- b'). The merged image (b") reveals that almost all the cells expressing $\mathrm{Ci}$ are glial cells. Scale bars: $50 \mu \mathrm{m}$.(c-d) Endogenous $\mathrm{Smo}$ and $\mathrm{Ci}$ are localised in the glial cells. Anterior $1 \mu \mathrm{M}$ section of a whole mount adult brain in which glial cells are specifically labelled with (c) mCD8::GFP (green channel) or with (d) anti-Draper (a glial receptor, green) and immunostained for Smo or $\mathrm{Ci}$ (red channel). Note that Smo and $\mathrm{Ci}$ staining are present in glial cells (dashed boxes), scale bars: $50 \mu \mathrm{m}$. (c'-d') Magnification of the region of the glia indicated by the dashed boxes. Scale Bars: $20 \mu \mathrm{m}$. (e-f) $h h$-expressing cells in the adult brain have neuronal identity. Projection pattern for Hh expressing cells (e-e"'). Cells positive for hh-Gal4 express membrane marker mCD8::GFP, (e-e"') images of $1 \mu \mathrm{m}$ sections for the forebrain, midbrain and hindbrain. (f) Expression of a nuclear GFP in hh-expressing cells of adult brain are positive for Elav (red channel), but not Repo (blue channel). Scale Bar: 50um. (f'-f') 5x magnification of the boxed regions shown in (f). The merge between $h h$-expressing cells (green) and antiElav immunostaining (red channel) is shown in the right panel. hh-expressing cells are indicated by white arrows. Scale bars: $5 \mu \mathrm{m}$. (Images consist of a $1 \mu \mathrm{m}$ section). (g-h") Representative confocal images of the PAM cluster cell bodies of control (g-g") and $h h$ mutant ( $h-h$ ") of aged adult fly brains, in which DA neurons are labelled with a nuclear GFP marker (GFP NLS in grey) and with anti-TH immunostaining (in red). Scale bars:10 $\mu \mathrm{m}$. (i-j) Quantifications of DA neuron number in the PAM cluster of young and aged flies. (k-n) $h h$ mutant and control brains with DA neurons immunostained with TH (in grey) in young $(\mathrm{k}, \mathrm{m})$ and aged flies $(\mathrm{l}, \mathrm{n})$. Arrows label the PAM DA neurons. (o) Quantifications of DA neuron volume in control and $h h$ mutant adult fly brains with $\mathrm{TH}$ immunostaining, in young $(\mathrm{Y})$, middle $(\mathrm{M})$ and aged flies $(\mathrm{A})$. For DA neuron number statistics the two tailed T-test was used and for DA neuron volume quantification two ways 
analysis of variance (ANOVA) test was used to compare the specified data points for $h h$ mutant and control samples $\left({ }^{* * *}, p<0.001 ;{ }^{* *}, p<0.01\right.$ and $\left.{ }^{*}, p<0.05\right)$.

Figure 2. Hh signalling affects adult lifespan determination and fitness

(a) Overexpression of $h h$ in $h h$-expressing cells - $h h$ OE ( $h h$ Gal4) - extends survival rate whereas $h h$ depletion drastically reduces it. Both combinations of $h h$ mutant alleles $\left(h h^{\text {ts }} / h h^{\text {ts }}\right.$ and $h h^{\text {ts }} / h h^{15}$ ) decrease the survival rate. (b) $h h$ overexpression in adult flies shows improved negative geotaxis ability during adult life compared to control flies whereas aged hhts/hhts mutant flies exhibit strong climbing defects. (c-d) The $h h$ dependent lifespan extension phenotype and improved fitness - hh OE (hh Gal4) - is abolished upon repression of $h$ h expression in neurons - $h$ h OE (elav-Gal80) - but not in glia (Table S2). (e-f) Expression of $h h$ in glutamatergic neurons of $h h$ mutant - $h h$ mutant $+h h$ OE (vGlut-Gal4) - efficiently rescues the short-lived phenotype of $h h$ mutants and the impaired locomotion phenotype. $p$ values refer to the degree of difference between the $h$ mutant and control/rescue samples as well as $h h \mathrm{OE}$ and equivalent control at median survival. Detailed genotypes, \% of changes in median survival and climbing activity, as well as corresponding $p$ values can be found for all figures in tables S2-3 and S5-6. For all lifespan studies the log rank test was utilized, to compare median lifespans of two specified data sets, and for all climbing activity assays (Table S2-S3 and Table S5S6), the two way ANOVA test was used to compare the specified data points ${ }^{* * *}, p<$ $0.001 ;{ }^{* *}, p<0.01$ and $\left.{ }^{*}, p<0.05\right)$.

Figure 3. Hh signaling in glial cells determines Hh mediated lifespan and is protective.

(a-b) Adult specific depletion of Smo (RNAi smo) or Ci (RNAi ci) in glia (repo-Gal4) but not in neurons (elav-Gal4) strongly reduces survival compared to the control. (b) Coxregression ${ }^{\mathrm{cr}}$ statistical analysis was used to compare control and RNAi lines and revealed no significant differences. (c-d) Expression of an activated form of $\mathrm{Ci}$ in the glia (ciact $\mathrm{OE}$, repo-Gal4) rescues the short survival time of $h h$ mutant, at $29^{\circ} \mathrm{C}$ well as (d) the impaired locomotion. (e-f) Overexpression of $c^{\text {act }}(\mathrm{e})$ or smo ${ }^{\text {act }}(\mathrm{f})$ in glial cells, extends lifespan (gi) Images of newly eclosed day 0 fly adult brains with TH immunostaining, (g) control, (h) 
$h h$ mutant, (i) $h h$ mutant expressing $c i^{\text {act }}$ in glia. (j-I) The expression of $c i^{\text {act }}$ (I) in the glia of $h$ m mutant flies, partially restores the volume of $\mathrm{TH}$ neurons exhibited in $h h$ mutant flies (k) (quantified in $\mathrm{m}$ ). Scale bars: $50 \mu \mathrm{m}$, Z-projections of entire adult brain. (n-o) neuronal $\mathrm{TH}(\mathrm{nTH})$ mRNA levels and dopamine brain concentration are significantly reduced in $h h$ mutant flies. Expression of $\mathrm{ci}^{\text {act }}$ in the glia potently restores both $(\mathrm{n}) \mathrm{nTH}$ mRNA expression and (o) brain dopamine to control levels. For lifespan studies the log rank test was utilized, and for climbing activity assays and DA neuron volume, the two-way ANOVA test was used $\left({ }^{* *}, p<0.001 ;{ }^{* *}, p<0.01\right.$ and ${ }^{*}, p<0.05$, see Tables S1-S3 and S5-S6). Analysis of mRNA levels TH expression and Dopamine levels differences between samples were determined by a two-tailed t-test and one way ANOVA test respectively $\left({ }^{* * *}, \mathrm{P}<0.001 ;{ }^{* *}, \mathrm{P}<0.01\right.$ and ${ }^{*}, \mathrm{P}<0.05$, see methods).

Figure 4. hh target chaperones maintain glial homeostasis, survival and fitness.

(a) List of protective factors tested in a qRT-PCR screen. mRNA levels significantly decreased in adult $h h$ mutant heads are highlighted in blue whereas mRNA levels of other chaperones and genes implicated in antioxidant defences and in lysosomal autophagy pathway (Fig. S4) show no significant difference. (b) The mRNA levels of $h s p 23, h s p 40$ and $h s p 68$ chaperones are all significantly decreased in 10-day-old $h h$ mutants (two-way t-test, $\left.p<0.001^{* * *}\right)$ and restored to close to or above control levels in $h h$ mutant flies expressing $\mathrm{Ci}^{\text {act }}$ in the glia. (c-d) Relative mRNA expression level of $h s p 40$ (c) and $h s p 68$ (d) at $25^{\circ} \mathrm{C}, 29^{\circ} \mathrm{C}$ and $37^{\circ} \mathrm{C}$ for day 12 control flies. No significant difference (One-way ANOVA, ns) was observed in mRNA levels of $h s p 40$ and $h s p 68$ between $25^{\circ} \mathrm{C}$ and $29^{\circ} \mathrm{C}$. (e-g) Day 15 hh mutant and control brains immunostained for Ubiquitin (Ubq, green) and Perlecan (Pcan, red in $\mathrm{f}, \mathrm{g}$ ). Scale bars $50 \mu \mathrm{m}$. (e'-g') Zooms of squared areas labelled in e-g. Scale Bars $30 \mu \mathrm{m}, 1 \mu \mathrm{M}$ section. Ubiquitin positive aggregates (blue arrows) are predominantly located in midbrain glial tracts immunostained with Pcan (f'). (h-k) Day 15 hh mutant (h) and control (i) brains and (j-k) hh mutant flies expressing $h s p 40$ (j) or $h s p 68$ (k) in glia, immunostained for anti-ubiquitin. Ubiquitin aggregates (blue arrows) are observed in $h h$ mutant adult brains (h). Expression of $h s p 40$ (j) or $h s p 68$ (k) in the glia of $h h$ mutants reduces the occurrence of aggregates. Quantification of Ubq signal intensity (I-l') were acquired from the equivalent boxed areas (i) in all samples, box 1: CB/OL 


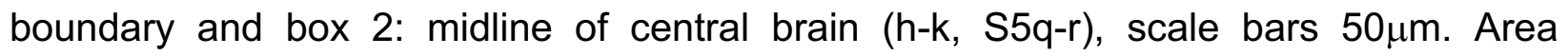
quantified for Ubq signal intensity: $31.62 \mu \mathrm{m} \times 31.62 \mu \mathrm{m}\left(1000 \mu \mathrm{m}^{2}\right)$. Two-way t-test used to analyse differences in Ubq signal intensity between $h h$ mutant and control samples in $\left.\mathrm{I}-\mathrm{I}, \mathrm{p}<0.001^{* * *}\right)$. Images (h-k) consists of a stack of $25 \mu \mathrm{M}(1 \mu \mathrm{M}$ per section). (m-n) Immunoblots showing pEIF2a levels in $h h$ mutant and $h$ h mutant flies expressing $h s p 68$ in the glia in day 12 adult head samples. (n) hh mutant flies exhibit a 2-fold increase in pEIF2a levels, which is rescued to control levels in $h h$ mutant samples with glial expression of $h s p 68$, ( $p<0.0001^{* * * *}$, one-way ANOVA with post-hoc Tukey).(o-p) Overexpression of $h s p 68$ in the glia strongly rescues the $h h$ mutant short-lived phenotype, log rank test, $p<0.0001$ (o) and (p) hh-defective locomotion - two- way ANOVA test, $p<0.001)$

Figure 5. Hh signalling in the glia has a protective function in a human Amyloid beta disease model.

(a-c) The shortened lifespan and debilitated locomotion that results from glial expression of human $\mathrm{A} \beta 42^{\mathrm{E} 693 \mathrm{G}}$ can be partially rescued by expression of $(\mathrm{a}, \mathrm{c})$ an activated form of Smo $\left(S m 0^{A c t}\right)$ or $(\mathrm{b}, \mathrm{c})$ hsp68 expression in the glia. The rescues are more potent in the human $A \beta 42{ }^{\mathrm{E} 693 \mathrm{G}}$ middle aged animals at the 20\% mortality rate (Table S2 and S3; as for locomotion assays in c, lifespan assays in a and b were performed in the same experiment and yellow and red curves separated for visualisation). For lifespan analysis log rank test utilised ( $p<0.0001)$, for climbing activity two-way Anova test was used, $p<0.001^{* * *}$. (d-e) Confocal section ( $5 \mu \mathrm{m}$ stack) of the optical lobe of 15 -day old flies expressing human $A \beta 42^{\mathrm{E} 693 \mathrm{G}}$ in the glia, (d) showing the presence of amyloid aggregates (demarcated by the dashed box), in the giant glial cells of the optic chiasm (glial cells labelled with GFP NLS in the merged image); in control specimens the amyloid aggregates are not discernable (e), Scale bars $20 \mu \mathrm{m}$. In order to quantify the presence of aggregates and to count cell number in the region of interest - ROI (dashed box), an area of $43.14 \mu \mathrm{m} \times 91.96 \mu \mathrm{m}$ was selected for the ROI (f). Blue arrows indicate the presence of A11 positively stained amyloid aggregates in flies overexpressing human $A \beta 42^{E 693 G}$ in the glia- red channel (f). The grey channel displays the presence of the 
amyloid (f'), as detected by the Amyloid Probe (AmyTracker ${ }^{\mathrm{TM}} 680$ ) and the cyan channel displays DAPI positive cells (f'), with the merge of all three channels also shown (f'”), Scale Bars $10 \mu \mathrm{m}$. Overexpression of either $(\mathrm{g}) \mathrm{Smo}^{A c t}(\mathrm{~h})$ or $h s p 68$ is able to reduce the presence of amyloid aggregates (j), (see Experimental procedures for amyloid aggregate quantification), and rescue the decreased cell number (g", h") observed in flies overexpressing human $A \beta 42^{\mathrm{E} 693 \mathrm{G}}(\mathrm{k})$. For both amyloid aggregate quantifications and cell number the two way t-test was used $\left(p<0.001^{* * *}\right)$

Figure 6 - Proposed model by which Hh signalling mediates glial proteostasis and neuroprotection

We put forward the following model: a) 1. the presence of the Hh signal 2. induces activation of the canonical $\mathrm{Hh}$ signalling pathway in the glia via $\mathrm{Ci}$ activation. 3. This regulates mRNA levels of downstream target chaperones in the glia, which maintain proteostasis and 4. maintains DA neuron integrity. b) 1 . Conversely in the absence of $\mathrm{Hh}$ signalling, $2 \mathrm{Ci}$ remains inactive in glia and 3 mRNA levels of downstream chaperones are decreased. Subsequently, glial proteostasis declines, leading to an increase in abnormal protein aggregation, characterised by an elevated level of Ubq positive aggregates. This proteotoxic environment in the glia is deleterious to lifespan and mobility of the organism and leads to 4 degeneration of DA neurons and a decrease in dopamine levels, potentially due to a reduction in neuroprotective factors produced in the glia.

Fig. S1. Location and expression of Hh signalling components in the adult brain, Related to Figure 1

(a-a") ci-expressing cells have glial identity (a) ci-lexA driving mCD8::GFP (green) colabelled with the glial markers repo-Gal4 5xmCD8 Cherry (red) and Draper (grey). Scale bars: $50 \mu \mathrm{m}$. Equivalent panels in a' represent $2 x$ magnified images of the anterior $(1 \mu \mathrm{m})$ section of the adult brain, demarcated by the boxed region. (a") The equivalent posterior section of the brain is shown in the lower panels. (b-e) Smo/Ci immunoreactivity is reduced upon RNAi knockdown of $s m o$ (c-c") and $c i$ in the glia (e-e"), compare to controls in $b$ and $d$, scale bars $20 \mu \mathrm{m}$. Magnification of the anterior $(1 \mu \mathrm{m})$ section of the brain (region outlined in upper panel) is shown in middle panel, scale bars $50 \mu \mathrm{m}$. Posterior 
$(1 \mu \mathrm{m})$ sections of control brains stained with anti-Smo (b") and anti-Ci (d"), and the corresponding RNAi knockdown are shown in b" and d". The left panels in a-a" show labelling of the glia using mCD8::GFP and the merge with the Smo immunostaining is shown in the right panels. (f) Schematic representation of the $h h-T 2 A-G a l 4$ trojan line. Projection pattern for $h$ h expressing cells. (g-g"') Cells positive for $h h-T 2 A-G a l 4$, expresses cytoplasmic GFP, (g-g"') images of $1 \mu \mathrm{m}$ sections of the forebrain, midbrain and hindbrain respectively). (h-i) $h$ h neurons are GABAergic and glutamatergic. The $h h-$ dsRed enhancer trap (which labels nuclei of $h$-expressing cells - left panels) overlaps (white arrows) with a nuclear GFP driven by either GAD-Gal4 or vGlut-Gal4 (middle panels - green channels). Genotypes: UAS: GFP NLS, hh-dsred/GAD-Gal4 and UAS: GFP NLS, hh-dsRed/vGlut-Gal4. Middle panels (h-i) and lower panels (i) are magnified images of single GABAergic and Glutamatergic cell body neurons positive for $h h$-dsRed (white arrows). Scale bars: $20 \mu \mathrm{m}$ and magnified images $5 \mu \mathrm{m}$ and $10 \mu \mathrm{m}$. CB and OL stand for central brain and optical lobe, respectively (Images consist of a $1 \mu \mathrm{m}$ section). (j) Table showing the subtypes of neuronal cells tested for overlaps with $h h$ positive cells.

\section{Fig S2. DA neuron loss in hh mutant flies, Related to Figure 1}

(a-d) Representative images of $h h$ mutant and control adult fly brains with DA neurons labelled with mCD8::GFP, in young $(a, c)$ and aged flies (b, d). Arrows label the PAM DA neurons. Scale bars: $50 \mu \mathrm{m}$, Z-projection stacks are of the entire adult brain. (e) Quantifications of DA neuron volume in the control and $h h$ mutant adult flies using a mCD8::GFP membrane marker. (f-g") Representative confocal images of the PAM cluster cell bodies of control (f-f') and $h$ h mutant (g-g") in young adult fly brains, in which DA neurons are labelled with a nuclear GFP marker (GFP NLS in grey) and with anti-TH

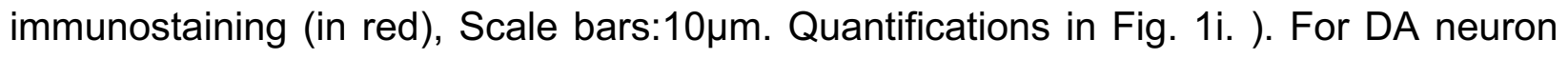
number and DA neuron volume quantification two-way ANOVA test was used to compare the specified data points for $h$ mutant and control samples $\left({ }^{* * *}, p<0.001\right)$.

Fig. S3. Hh signalling in GABAergic neurons partially rescues the $h \boldsymbol{h}$ mutant shortened lifespan and defective locomotion, Related to Figure 2. 
(a) Overexpression of $h h$ in GABAergic neurons partially rescues the $h h$ mutant shortened survival phenotype and (b) the impaired locomotion phenotype. (b) $h$ h mutant male flies $\left(h h^{t s 2} / h h^{t s 2}\right)$ exhibit a reduced survival time. (d-j) Survival time of UAS and Gal4 control lines: (d) hh-Gal4, Gal80 $/$ t+; (e) UAS:hhM4; (f) UAS:ci ${ }^{\text {act } /+; ~(g) ~ U A S: s m o ~ d s R N A /+; ~(h) ~}$ UAS:ci dsRNA/+; (i) UAS:Dcr2 (II)/+; (j) UAS:Dcr2 (III)/+. All compared to control $w^{1118}$ (c, $\mathrm{d}, \mathrm{f}-\mathrm{j}$ ) or UAS-GFP NLS flies (e). (k) Expression of an activated form of Ci in the glia (cict $\mathrm{OE}$, repo-Gal4) rescues the short survival time of $h h$ mutant at $25^{\circ} \mathrm{C}$. For lifespan analysis log rank test was utilised $(p<0.0001)$, for climbing activity two-way anova test was used, $\mathrm{p}<0.001^{* * *}$.

Fig S4. Stimulation of Hh signalling and expression of Hh target chaperones in the glia potently rescues the $\boldsymbol{h} h$ mutant shortened lifespan, Related to Figure 3 and 4. (a-a") Three independent experiments performed in triplicate, showing that overexpression of $\mathrm{ci}^{\text {act }}$ rescues the $h h$ mutant short-lived phenotype. Overexpression of hsp68 (b-b") and hsp40 (c-c") potently rescue the hh mutant short-lived phenotype, (log rank test $-p<0.0001)$ whereas no rescue was observed with overexpression of $h s p 23$ (log rank test - ns) (d-d"). Note that longevity curves for $h s p 68$ and $h s p 23$ were run in parallel with the same controls. For lifespan analysis log rank test was utilised and $h h$ mutant samples were compared to $h h$ mutants expressing the specified chaperone in the glia, see Table S2 and Table S5.

Fig. S5. Proteostasis defects in $h \boldsymbol{h}$ mutant flies, Related to Figure 4.

(a) mRNA expression level of Hh signalling pathway components in adult heads. Both $h h$ and ptc mRNA levels were substantially decreased in $h h$ mutant samples compared with age-matched controls ( $p<0.001^{* * *}$, two tailed t-test) (b-d) Among several tested genes, the mRNA levels of genes implicated in antioxidant defences (b), the lysosomal autophagy pathway (c) and chaperones (d) exhibit no significant difference except for the ones presented in figure 4b. Each experiment consisted of 4-5 independent biological replicates. The genes and the pairs of primers used for the analysis are listed in Methods. (e-l") Representative images of day 10 hh mutant $(n=10)$ and control fly brains $(n=10)$ with glial cells labelled with mCD8::GFP (green channel). In all analysed $h h$ mutant samples 
we observed abnormal accumulations of GFP in the glia, which also contain elevated level of ubiquitin (Ubq) staining, (indicated by blue arrows - red channel) in both anterior (e-e") and posterior brain regions (i-i"). This phenomenon was not observed in the equivalent aged matched control samples in either the anterior ( $g-g$ ") or posterior brain regions (k-k"). $1 \mu \mathrm{m}$ section, scale bars $50 \mu \mathrm{m}$. The equivalent magnified image of the region of interest (marked by the blue dashed rectangle) are displayed in the adjacent panels (f-f", h-h", j-j" and I-l"), respectively. $1 \mu \mathrm{m}$ stack, scale bars $30 \mu \mathrm{m}$. (m-n") Control flies with glial cells labelled with mCD8::GFP (green channel) in the midbrain region $\mathrm{m}$ ), immunostained with anti-Perlecan (Pcan, red in m'), merged channels (m"). $1 \mu \mathrm{m}$ section, scale bars $50 \mu \mathrm{m}$. ( $\mathrm{n}$ ) Magnification of the region of interest (demarcated by the blue dashed rectangle in $\mathrm{m}$ ) of the glial tracts in the adult midbrain are displayed in the adjacent panels (n-n"). Stack of $2 \times 1 \mu \mathrm{m}$ sections, scale bars $50 \mu \mathrm{m}$. (0) mCD8-GFP driven in ciexpressing cells immunostained with Pcan (o') and merged image in o", scale bars 50 $\mu \mathrm{m}$. Magnification of the region of interest (demarcated by the white dashed rectangle in o) are displayed in the adjacent panels (p-p"). Stack of $2 \times 1 \mu \mathrm{m}$ sections, scale bars 30 $\mu \mathrm{m}$. (q-r) Images of Day 0 hh mutant and control brains immunostained for Ubiquitin (Ubq, green), scale bars $50 \mu \mathrm{m}$, quantifications, (s, see Fig. 4l-l'), two-tailed t-test (ns). (t) Representative Immunoblot showing pEIF2a levels in $h$ h overexpressing day 30 adult head samples and aged matched controls. (u) hh overexpressing samples exhibit a $32 \%$ reduction in pEIF2a levels compared to age-matched control specimens, $\left(p<0.005^{* *}\right.$, twotailed t-test).

Fig. S6. Detection of Amyloid aggregates in adult brains of flies expressing the human $\mathrm{A} \beta 42^{\mathrm{E} 693 \mathrm{G}}$ variant in glial cells, Related to Figure 5.

Representative images of $1 \mu \mathrm{m}$ sections for the forebrain (a-a"'), hindbrain (c-c"') and midbrain (e-e"', $5 \mu \mathrm{m}$ section) expressing the human A $\beta 42{ }^{\mathrm{E} 693 G}$ variant along with GFP NLS (green channel) in the glia and stained for DAPI (cyan channel), along with the merged channel in 22 day old specimens. The equivalent age-matched control samples expressing only GFP NLS in the glia are also shown: forebrain (b-b"'); hindbrain (d-d"') and midbrain (f-f'") sections. Throughout all sections of the adult brain amyloid aggregates (indicated by blue arrows) can be observed in the samples expressing human 
$\mathrm{A} \beta 42^{\mathrm{E} 693 \mathrm{G}}$ in the glia, whereas this is not the case for the control specimens, immunostained with the A11 amyloid aggregate specific antibody, Scale Bars: $50 \mu \mathrm{m}$; The demarcated dashed box (ROI) in e, encompasses the giant glial cells of the optic chiasma and the magnified images (g-g"') reveal the presence of amyloid aggregates indicated by the blue arrows (g), whereas in the equivalent control specimens (h-h"') such aggregated structures could not be detected (h). Samples expressing human $A \beta 42{ }^{\mathrm{E} 693 \mathrm{G}}$ in the glia also exhibited enlarged cells with a swollen morphology (red arrows) and a reduction in cell numbers in the ROI compared to control samples. Scale Bars: 10 $\mu \mathrm{m}$. (i-j) Confocal section ( $5 \mu \mathrm{m}$ stack) of the optical lobe of 15 day old flies expressing human $A \beta 42$ E693G along with $\mathrm{Smo}^{\mathrm{Act}}$ (i) or Hsp68 (j) in the glia, Scale Bars $20 \mu \mathrm{m}$. In order to quantify the surface area of amyloid aggregates and cell number in the region of interest - ROI (dashed box), an area of $43.14 \mu \mathrm{m} \times 91.96 \mu \mathrm{m}$ was selected (see Figure $5 \mathrm{~g}-\mathrm{h}$, for selected ROls).

\section{Table S1}

A summary of the DA neuron volume data and statistical analysis for all the relevant genotypes shown in the text. (Statistical comparisons between age-matched samples for young, middle aged and old flies are colour coded blue, green and yellow respectively).

\section{Table S2}

Summary of statistical analysis/median and maximum lifespan values for all relevant genotypes mentioned in the text.

\section{Table S3.}

A summary of the climbing ability and a statistical analysis for all the relevant genotypes mentioned in the text.

\section{Table S4.}

Displays the short-hand notation and the corresponding full genotype used in both the lifespan and locomotion analysis. 


\section{Table S5.}

Shows the data sets that were compared when calculating the $p$ value in the lifespan studies.

\section{Table S6.}

Displays the data sets that were compared when calculating the p value in the locomotion assays.

\section{REFERENCES}

Ahn, S. and Joyner, A. L. (2005). In vivo analysis of quiescent adult neural stem cells responding to Sonic hedgehog. Nature 437, 894-897.

Akimoto, A., Wada, H. and Hayashi, S. Enhancer trapping with a red fluorescent protein reporter in Drosophila (2005). Developmental Dynamics 233, 993-997

Álvarez-Buylla A, Ihrie RA. (2014) Sonic hedgehog signaling in the postnatal brain. Semin Cell Dev Biol;33:105-11. Review.

Amankulor NM, Hambardzumyan D, Pyonteck SM, Becher OJ, Joyce JA, Holland EC. (2009). Sonic hedgehog pathway activation is induced by acute brain injury and regulated by injury-related inflammation. J Neurosci;29(33):10299-308

Awasaki, T. and Lee, T. New tools for the analysis of glial cell biology in Drosophila. (2011). Glia 59, 1377-1386.

Bambakidis NC, Wang X, Lukas RJ, Spetzler RF, Sonntag VK, Preul MC. (2010). Intravenous hedgehog agonist induces proliferation of neural and oligodendrocyte precursors in rodent spinal cord injury. Neurosurgery. 67(6):1709-15.

Bambakidis NC, Petrullis M, Kui X, Rothstein B, Karampelas I, Kuang Y, Selman WR, LaManna JC, Miller RH. (2012). Improvement of neurological recovery and stimulation of 
neural progenitor cell proliferation by intrathecal administration of Sonic hedgehog. J Neurosurg. 116(5):1114-20

Beachy PA, Karhadkar SS, Berman DM. (2004) Tissue repair and stem cell renewal in carcinogenesis. Nature 432(7015):324-31. Review.

Biehs, B., Kechris, K., Liu, S. and Kornberg, T (2010). Hedgehog targets in the Drosophila embryo and the mechanisms that generate tissue-specific outputs of Hedgehog signaling. Development 137, 3887-3898

Briscoe, J. and Thérond, P. (2013). The mechanisms of Hedgehog signalling and its roles in development and disease. Nature Reviews Molecular Cell Biology 14, 418-431

Chechneva OV, Mayrhofer F, Daugherty DJ, Krishnamurty RG, Bannerman P, Pleasure DE, Deng W. (2014). A Smoothened receptor agonist is neuroprotective and promotes regeneration after ischemic brain injury. Cell Death Dis 5:e1481.

Croset V, Treiber CD, Waddell S (2018). Cellular diversity in the Drosophila midbrain revealed by single-cell transcriptomics. Elife. 7. pii: e34550.

Curran T. (2018). Reproducibility of academic preclinical translational research: lessons from the development of Hedgehog pathway inhibitors to treat cancer. Open Biol. 8(8). pii: 180098. Review.

D'Andrea MR, Nagele RG, Wang HY, Peterson PA, Lee DH. (2001) Evidence that neurones accumulating amyloid can undergo lysis to form amyloid plaques in Alzheimer's disease. Histopathology. 38(2):120-34.

Davie K, Janssens J, Koldere D, De Waegeneer M, Pech U, Kreft Ł, Aibar S, Makhzami S, Christiaens V, Bravo González-Blas C, Poovathingal S, Hulselmans G, Spanier KI, Moerman T, Vanspauwen B, Geurs S, Voet T, Lammertyn J, Thienpont B, Liu S, 
Konstantinides N, Fiers M, Verstreken P, Aerts S. (2018). A Single-Cell Transcriptome Atlas of the Aging Drosophila Brain. Cell;174(4):982-998.

De Luca M, Roshina NV, Geiger-Thornsberry GL, Lyman RF, Pasyukova EG, Mackay TF. (2003)Dopa decarboxylase (Ddc) affects variation in Drosophila longevity. Nat Genet. 34(4):429-33

Diao F, Ironfield H, Luan H, Diao F, Shropshire WC, Ewer J, Marr E, Potter CJ, Landgraf M, White BH.(2015) Plug-and-play genetic access to drosophila cell types using exchangeable exon cassettes. Cell Rep.10(8):1410-21.

Echelard Y, Epstein DJ, St-Jacques B, Shen L, Mohler J, McMahon JA, McMahon AP. (1993) Sonic hedgehog, a member of a family of putative signaling molecules, is implicated in the regulation of CNS polarity. Cell. 75(7):1417-30.

Edenharter O, Schneuwly S,. Navarro JA (2018). Mitofusin-Dependent ER Stress Triggers Glial Dysfunction and Nervous System Degeneration in a Drosophila Model of Friedreich's Ataxia. Front Mol Neurosci. 11: 38.

Eitan E, Petralia RS, Wang YX, Indig FE, Mattson MP, Yao PJ. (2016). Probing extracellular Sonic hedgehog in neurons. Biol Open. 5(8):1086-92.

Evans CG, Wisén S, Gestwicki JE. (2006). Heat shock proteins 70 and 90 inhibit early stages of amyloid beta-(1-42) aggregation in vitro. J Biol Chem. 281(44):33182-91.

Farmer WT, Abrahamsson T, Chierzi S, Lui C, Zaelzer C, Jones EV, Bally BP, Chen GG, Théroux JF, Peng J, Bourque CW, Charron F, Ernst C, Sjöström PJ, Murai KK. (2016) Neurons diversify astrocytes in the adult brain through sonic hedgehog signaling. Science. 351(6275):849-54.

Feany, M. and Bender, W (2000). A Drosophila model of Parkinson's disease. Nature 404, 394-398 
Ferent J, Cochard L, Faure H, Taddei M, Hahn H, Ruat M, Traiffort E. (2014). Genetic activation of Hedgehog signaling unbalances the rate of neural stem cell renewal by increasing symmetric divisions. Stem Cell Reports. 3(2):312-23.

Friggi-Grelin, F., Iché, M. and Birman, S. (2003) Tissue-specific developmental requirements of Drosophila tyrosine hydroxylase isoforms. Genesis 35, 175-184

Garcia, A., Petrova, R., Eng, L. and Joyner, A. (2010). Sonic Hedgehog Regulates Discrete Populations of Astrocytes in the Adult Mouse Forebrain. Journal of Neuroscience $30,13597-13608$

Gonzalez-Reyes LE, Verbitsky M, Blesa J, Jackson-Lewis V, Paredes D, Tillack K, Phani S, Kramer ER, Przedborski S, Kottmann AH. (2012) Sonic hedgehog maintains cellular and neurochemical homeostasis in the adult nigrostriatal circuit. Neuron.75(2):306-19

Halliday M, Hughes D, Mallucci GR. (2017). Fine-tuning PERK signaling for neuroprotection. J Neurochem.142(6):812-826. Review.

Hardie, S. L., and Hirsh, J. (2006) An improved method for the separation and detection of biogenic amines in adult Drosophila brain extracts by high performance liquid chromatography. Journal of Neuroscience Methods, 153(2), 243-249.

Hoshino T, Murao N, Namba T, Takehara M, Adachi H, Katsuno M, Sobue G, Matsushima T, Suzuki T, Mizushima T. (2011) Suppression of Alzheimer's disease-related phenotypes by expression of heat shock protein 70 in mice. J Neurosci. 31(14):5225-34.

Huang SS, Cheng H, Tang CM, Nien MW, Huang YS, Lee IH, Yin JH, Kuo TB, Yang CC, Tsai SK, Yang DI. (2013). Anti-oxidative, anti-apoptotic, and pro-angiogenic effects mediate functional improvement by sonic hedgehog against focal cerebral ischemia in rats. Exp Neurol. 247:680-8. 
Ingham PW (2018). From Drosophila segmentation to human cancer therapy. Development. 145(21). Review.

Jackrel ME, Shorter J. (2017). Protein-Remodeling Factors As Potential Therapeutics for Neurodegenerative Disease.Front Neurosci. 11:99. Review.

Ji H, Miao J, Zhang X, Du Y, Liu H, Li S, Li L. (2012). Inhibition of sonic hedgehog signaling aggravates brain damage associated with the down-regulation of Gli1, Ptch1 and SOD1 expression in acute ischemic stroke. Neurosci Lett. 506(1):1-6

Jin Y, Raviv N, Barnett A, Bambakidis NC, Filichia E, Luo Y. (2015). The shh signaling pathway is upregulated in multiple cell types in cortical ischemia and influences the outcome of stroke in an animal model. PLoS One.10(4).

Jonson M, Nyström S, Sandberg A, Carlback M, Michno W, Hanrieder J, Starkenberg A, Nilsson KPR, Thor S, Hammarström P. (2018) Aggregated A $\beta 1-42$ Is Selectively Toxic for Neurons, Whereas Glial Cells Produce Mature Fibrils with Low Toxicity in Drosophila. Cell Chem Biol. 25(5):595-610.

Kounatidis I, Chtarbanova S, Cao Y, Hayne M, Jayanth D, Ganetzky B, Ligoxygakis P. NF-kB Immunity in the Brain Determines Fly Lifespan in Healthy Aging and Age-Related Neurodegeneration. Cell Rep. 19(4):836-848.

Lai K, Kaspar BK, Gage FH, Schaffer DV. (2003) Sonic hedgehog regulates adult neural progenitor proliferation in vitro and in vivo. Nat Neurosci. 6(1):21-7.

Lake BB, Chen S, Sos BC, Fan J, Kaeser GE, Yung YC, Duong TE, Gao D, Chun J, Kharchenko PV, Zhang K (2018). Integrative single-cell analysis of transcriptional and epigenetic states in the human adult brain. Nat Biotechnol. 36(1): 70-80. 
Liao S, Broughton S, Nässel DR (2017). Behavioral Senescence and Aging-Related Changes in Motor Neurons and Brain Neuromodulator Levels Are Ameliorated by Lifespan-Extending Reproductive Dormancy in Drosophila. Front Cell Neurosci, 11:111

Lindquist S, Craig EA. The heat-shock proteins. (1988) Annu Rev Genet.; 22:631-77. Review.

Magrané J, Smith RC, Walsh K, Querfurth HW. (2004). Heat shock protein 70 participates in the neuroprotective response to intracellularly expressed beta-amyloid in neurons. $J$ Neurosci.24(7):1700-6.

Martinez JA, Kobayashi M, Krishnan A, Webber C, Christie K, Guo G, Singh V, Zochodne DW. (2015) Intrinsic facilitation of adult peripheral nerve regeneration by the Sonic hedgehog morphogen. Exp Neurol. 271:493-505.

Miller D, Hannon C, Ganetzky B (2012). A mutation in Drosophila Aldolase causes temperature-sensitive paralysis, shortened lifespan, and neurodegeneration. J Neurogenet. 26(3-4):317-27

Mohler, J. (1988) Requirements for hedgehog, a segmental polarity gene, in patterning larval and adult cuticle of Drosophila. Genetics 120, 1061-72.

Morrow G. and Tanguay R. M. (2015). Drosophila small heat shock proteins: an update on their features and functions. In The Big Book of sHSPs (ed. Tanguay R. M. and Hightower L. E.), pp. 579- 606. Switzerland: Springer International.

Navarro JA, Heßner S, Yenisetti SC, Bayersdorfer F, Zhang L, Voigt A, Schneuwly S, Botella JA. (2014); Analysis of dopaminergic neuronal dysfunction in genetic and toxininduced models of Parkinson's disease in Drosophila. J Neurochem 131(3):369-82. 
Navarro JA, Ohmann E, Sanchez D, Botella JA, Liebisch G, Moltó MD, Ganfornina MD, Schmitz G, Schneuwly S. (2010) ; Altered lipid metabolism in a Drosophila model of Friedreich's ataxia. Hum Mol Genet. 19(14):2828-40.

Nezis IP, Simonsen A, Sagona AP, Finley K, Gaumer S, Contamine D, Rusten TE, Stenmark H, Brech A. (2008). Ref(2)P, the Drosophila melanogaster homologue of mammalian p62, is required for the formation of protein aggregates in adult brain. $\mathrm{J}$ Cell Biol. 180(6):1065-71

Nilsberth C, Westlind-Danielsson A, Eckman CB, Condron MM, Axelman K, Forsell C, Stenh C, Luthman J, Teplow DB, Younkin SG, Näslund J, Lannfelt L. (2001) The 'Arctic' APP mutation (E693G) causes Alzheimer's disease by enhanced Abeta protofibril formation. Nat Neurosci. 4(9):887-93.

Nüsslein-Volhard, C., Wieschaus, E. and Kluding, H. (1994) Mutations affecting the larval cuticle in Drosophila melanogaster: zygotic loci on the second chromosome. Roux's Arch. Dev. Biol. 193, 267-282.

Okuda H, Tatsumi K, Morita-Takemura S, Nakahara K, Nochioka K, Shinjo T, Terada Y, Wanaka A. (2016). Hedgehog Signaling Modulates the Release of Gliotransmitters from Cultured Cerebellar Astrocytes. Neurochem Res.;41(1-2):278-89.

Paaby AB and Schmidt PS (2009). Dissecting the genetics of longevity in Drosophila melanogaster. Fly (Austin), 3(1):29-38.

Palm W, Swierczynska MM, Kumari V, Ehrhart-Bornstein M, Bornstein SR, Eaton S. Secretion and signaling activities of lipoprotein-associated hedgehog and non-sterolmodified hedgehog in flies and mammals (2013). PLoS Biol.;11(3):e1001505. 
Palma V, Lim DA, Dahmane N, Sánchez P, Brionne TC, Herzberg CD, Gitton Y, Carleton A, Alvarez-Buylla A, Ruiz i Altaba A. (2005). Sonic hedgehog controls stem cell behavior in the postnatal and adult brain. Development. 132(2):335-44.

Petrova R, Joyner AL (2014). Roles for Hedgehog signaling in adult organ homeostasis and repair. Development. 141(18):3445-57. Review.

Pitter KL, Tamagno I, Feng X, Ghosal K, Amankulor N, Holland EC, Hambardzumyan D. (2014). The SHH/Gli pathway is reactivated in reactive glia and drives proliferation in response to neurodegeneration-induced lesions. Glia.62(10):1595-607

Purice, M., Speese, S. and Logan, M (2016). Delayed glial clearance of degenerating axons in aged Drosophila is due to reduced PI3K/Draper activity. Nature Communications $7,12871$.

Ranieri N, Ruel L, Gallet A, Raisin S and Thérond PP. (2012) Distinct phosphorylations on kinesin costal-2 mediate differential hedgehog signaling strength. Dev Cell, 22(2):27994.

Riemensperger T, Issa AR, Pech U, Coulom H, Nguyễn MV, Cassar M, Jacquet M, Fiala A, Birman S. (2013) A single dopamine pathway underlies progressive locomotor deficits in a Drosophila model of Parkinson disease. Cell Rep, 5(4):952-60.

Rodrigue KM, Kennedy KM, Park DC. (2009). Beta-amyloid deposition and the aging brain. Neuropsychol Rev. 19(4):436-50. Review.

Simonsen A, Cumming RC, Brech A, Isakson P, Schubert DR, Finley KD. (2008). Promoting basal levels of autophagy in the nervous system enhances longevity and oxidant resistance in adult Drosophila. Autophagy. 4(2):176-84 
Sirko S, Behrendt G, Johansson PA, Tripathi P, Costa M, Bek S, Heinrich C, Tiedt S, Colak D, Dichgans M, Fischer IR, Plesnila N, Staufenbiel M, Haass C, Snapyan M, Saghatelyan A, Tsai LH, Fischer A, Grobe K, Dimou L, Götz M. (2013) Reactive glia in the injured brain acquire stem cell properties in response to sonic hedgehog Cell Stem Cell. 12(4):426-39

Strigini M and Cohen SM (1997). A Hedgehog activity gradient contributes to AP axial patterning of the Drosophila wing. Development.124(22):4697-705.

Stork T, Bernardos R, Freeman MR. (2012) Analysis of glial cell development and function in Drosophila. Cold Spring Harb Protoc. 2012(1):1-17. Review.

Sun X, Komatsu T, Lim J, Laslo M, Yolitz J, Wang C, Poirier L, Alberico T, Zou S. (2012) Nutrient-dependent requirement for SOD1 in lifespan extension by protein restriction in Drosophila melanogaster. Aging Cell. 11(5):783-93

Takeuchi T, Suzuki M, Fujikake N, Popiel HA, Kikuchi H, Futaki S, Wada K, Nagai Y (2015) Intercellular chaperone transmission via exosomes contributes to maintenance of protein homeostasis at the organismal level. Proc Natl Acad Sci U S A. 112(19).

Thanos PK, Hamilton J, O'Rourke JR, Napoli A, Febo M, Volkow ND, Blum K, Gold M (2016) Dopamine D2 gene expression interacts with environmental enrichment to impact lifespan and behavior. Oncotarget. 7(15):19111-23.

Thomas JL, Morgan GW, Dolinski KM, Thummel R. (2018). Exp Eye Res. 166:106-115

Tower J. (2011) Heat shock proteins and Drosophila aging. Exp Gerontol. 46(5):355-62. Review. 
Ugbode CI, Smith I, Whalley BJ, Hirst WD, Rattray M (2017). Sonic hedgehog signalling mediates astrocyte crosstalk with neurons to confer neuroprotection. J Neurochem. 142(3):429-443.

Veeraraghavalu K, Zhang C, Zhang X, Tanzi RE, Sisodia SS. (2014) Age-dependent, non-cell-autonomous deposition of amyloid from synthesis of $\beta$-amyloid by cells other than excitatory neurons. J Neurosci. 34(10):3668-73.

Volkenhoff A, Weiler A, Letzel M, Stehling M, Klämbt C, Schirmeier S.(2015). Glial Glycolysis Is Essential for Neuronal Survival in Drosophila. Cell Metab. 22(3):437-47.

Wang, G., Wang, B. and Jiang, J (1999). Protein kinase A antagonizes Hedgehog signaling by regulating both the activator and repressor forms of Cubitus interruptus. Genes \& Development 13, 2828-2837

Wang MC, Bohmann D and Jasper H (2005). JNK extends life span and limits growth by antagonizing cellular and organism-wide responses to insulin signaling. Cell.121(1):11525.

Wu, J. and Luo, L. (2006) A protocol for dissecting Drosophila melanogaster brains for live imaging or immunostaining. Nature Protocols 1, 2110-2115

Yao PJ, Petralia RS, Mattson MP. (2016) Sonic Hedgehog Signaling and Hippocampal Neuroplasticity. Trends Neurosci;39(12):840-850. Review.

Yao PJ, Manor U, Petralia RS, Brose RD, Wu RT, Ott C, Wang YX, Charnoff A, LippincottSchwartz J, Mattson MP. (2017) Sonic hedgehog pathway activation increases mitochondrial abundance and activity in hippocampal neurons. Mol Biol Cell. 2017 28(3):387-395. 
Yin JA, Gao G, Liu XJ, Hao ZQ, Li K, Kang XL, Li H, Shan YH, Hu WL, Li HP, Cai SQ. (2017). Genetic variation in glia-neuron signalling modulates ageing rate._Nature. 551(7679):198-203.

Zarouchlioti C, Parfitt DA, Li W, Gittings LM, Cheetham ME. (2018). DNAJ Proteins in neurodegeneration: essential and protective factors. Philos Trans R Soc Lond B Biol Sci. 373(1738). pii: 20160534. Review

Zhang C, Williams EH, Guo Y, Lum L, Beachy PA. (2004) Extensive phosphorylation of Smoothened in Hedgehog pathway activation. Proc Natl Acad Sci U S A. 2004 Dec 28;101(52):17900-7. Epub 2004 Dec 14.

Zhang Y, Dong W, Guo S, Zhao S, He S, Zhang L, Tang Y, Wang H. (2014) Neurol Sci. 35(12):1931-40 Lentivirus-mediated delivery of sonic hedgehog into the striatum stimulates neuroregeneration in a rat model of Parkinson disease.

\section{Acknowledgments}

We thank all "fly" members of the IBV Institute, and Tamas Matusek, Laurence StacciniLavenant and Caterina Novelli for additional help. We also thank Julien Marcetteau, Pierre Léopold, Florence Besse, Caroline Médioni, Arnaud Hubstenberger, Hervé Tricoire, Thomas Préat, Pauline Spéder, Laure Bally-Cuif and Catherine Rabouille for critical analysis of the manuscript. AR held a Ligue Nationale Contre le Cancer fellowship. This work was supported by grants from Ligue National and Contre le Cancer "Equipe labellisée 2016', the LABEX SIGNALIFE (ANR-11-LABX-0028-01), the ANR (ANR-15CE13-0002-01 and ANR-17-C814-0041-01) to P.P.T, the Federation de la Recherche sur le Cerveau to S.B.

Competing financial interests:

The authors declare no competing financial interests.

\section{Authors contributions}


A. R. and P. P. T. conceived the experiments and wrote the manuscript. J. N. and M. R. conducted the experiments on Figures 1a-b, 3n, 4a-d, 4m-n S1f-g"', S5a-d and S5t-u. S.B. and AH conducted experiment on Figure 30. A. R. conducted all other experiments. All authors participated in the critical analysis of the manuscript. 


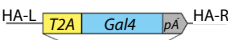

bioRxiv preprint doi: https://doi.org/10.1101/2020.02.05.935585; this (wifrch was not certified by peer-review) is the author/fund

$\square$ untranslated $\square$ coding CRISPR/Cas target site
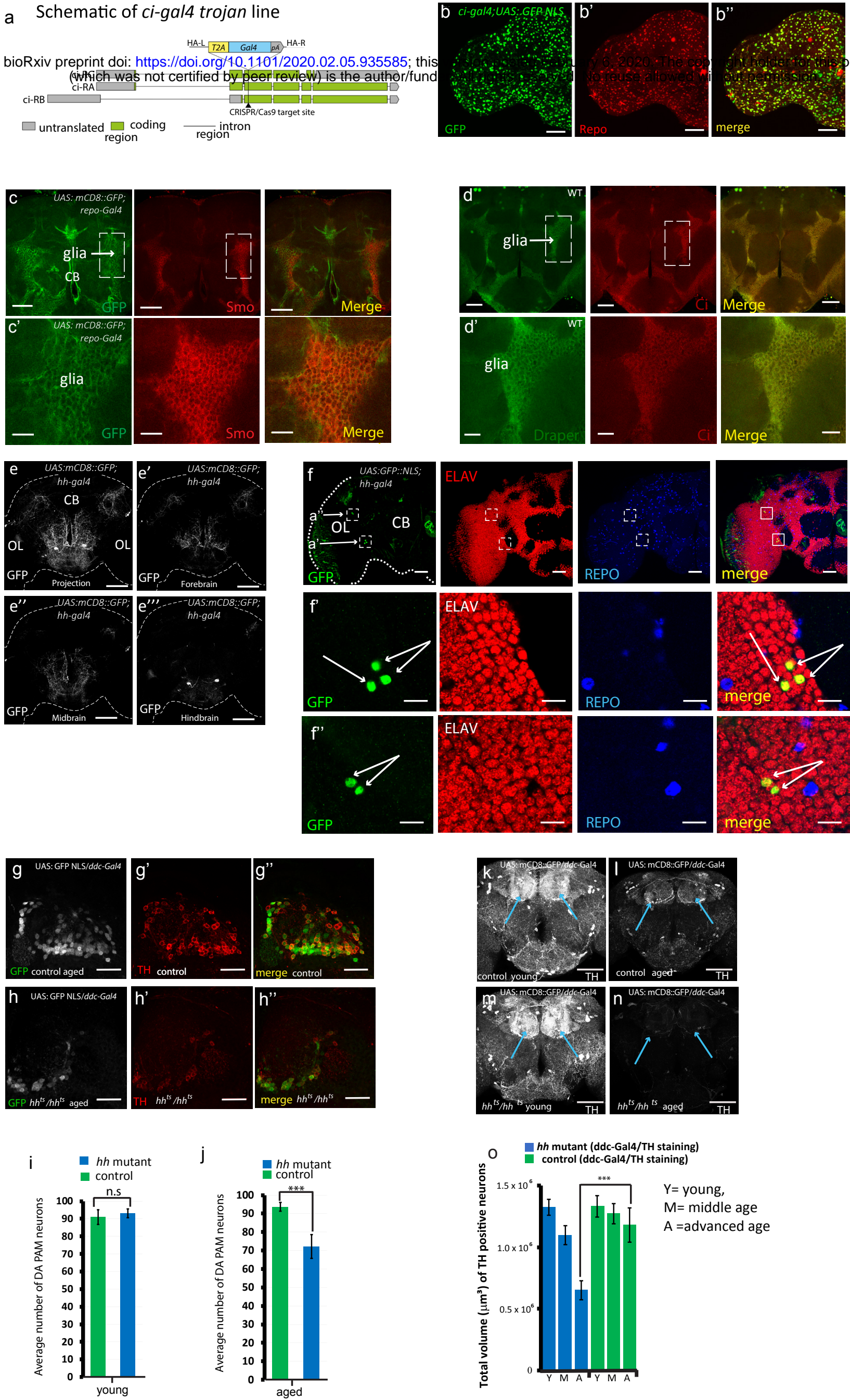


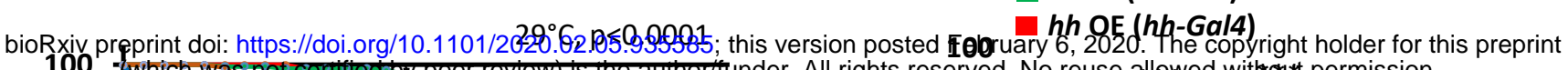

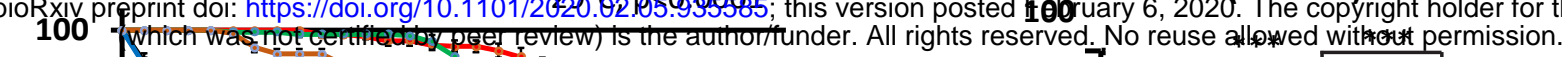
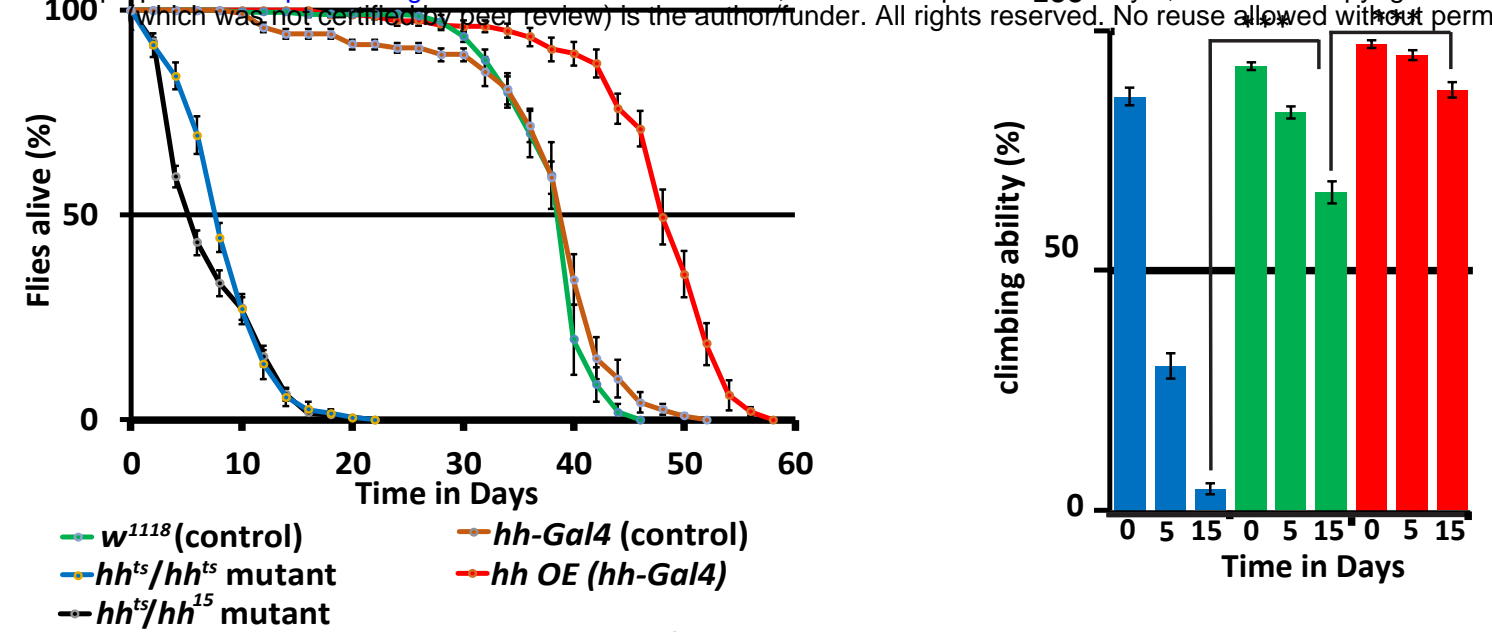

C

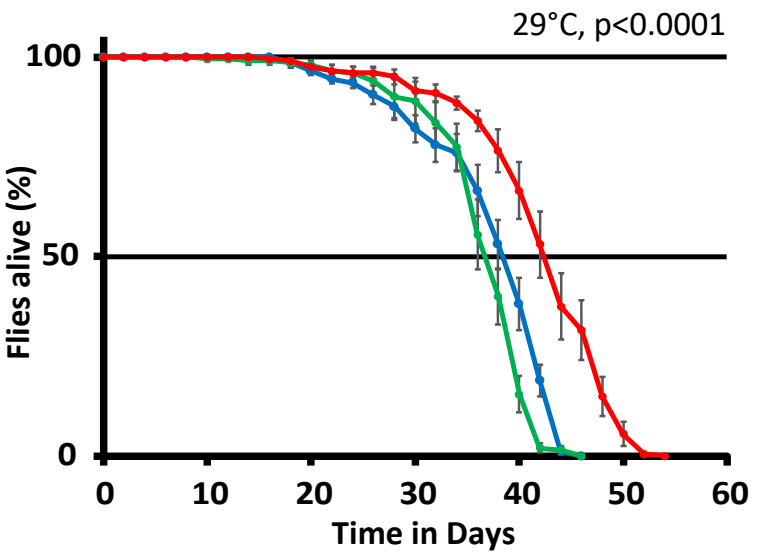

$\rightarrow$ hh-Gal4 (control) $\quad-$ hh OE (hh-Gal4)

$\rightarrow$ hh OE + elav-Gal80

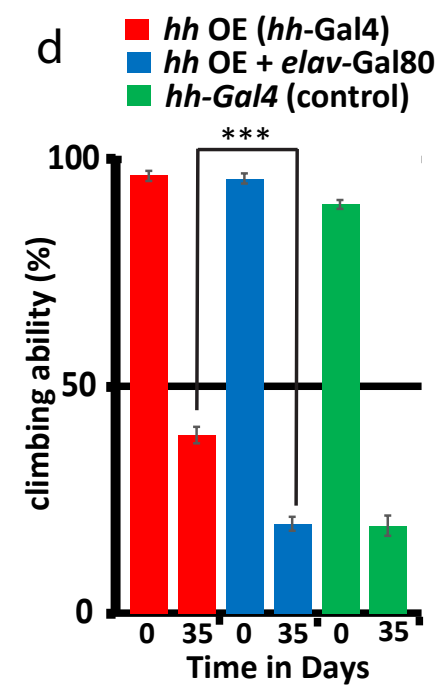

e

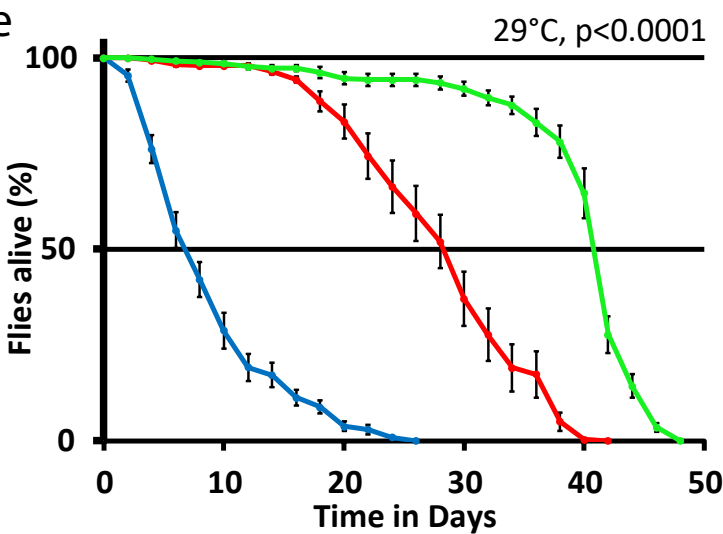

- hh mutant (vGlut-Gal4)

- vGlut-Gal4 (control)

- hh mutant + hh OE (vGlut-Gal4)

f $\quad h$ h mutant (vGlut-Gal4)

vGlut-Gal4 (control)

bh mutant + hh OE (vGlut-Gal4)

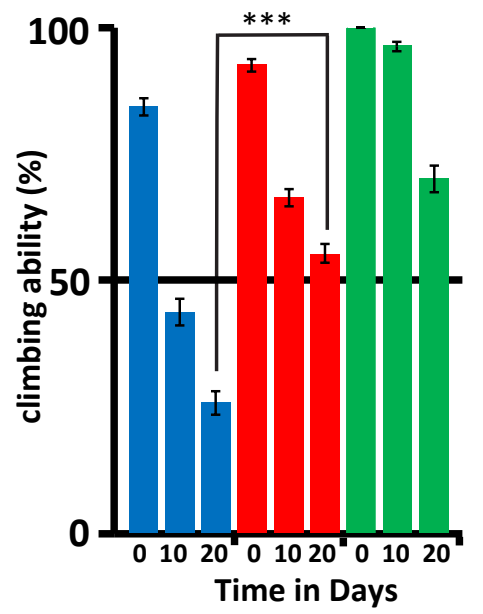




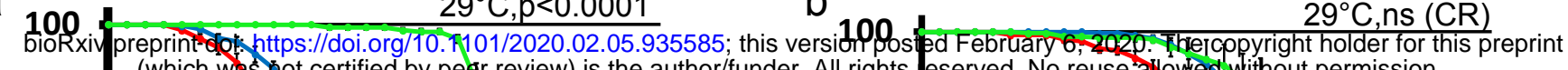

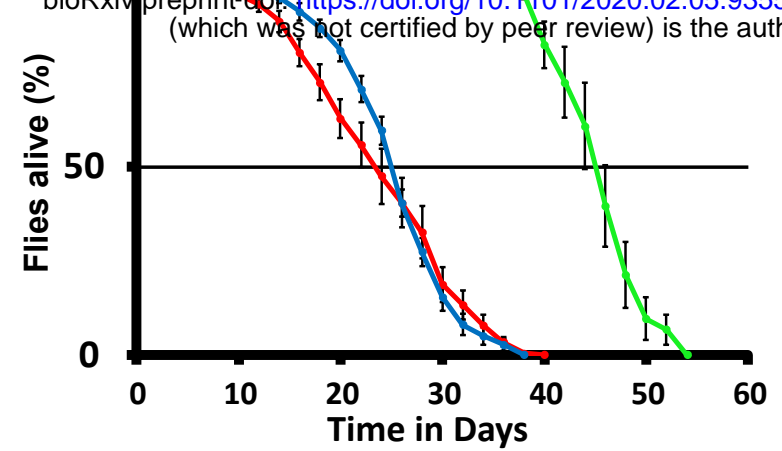

- RNAi smo (repo-Gal4) -control (repo-Gal4)

$\rightarrow$ RNAi ci (repo-Gal4)

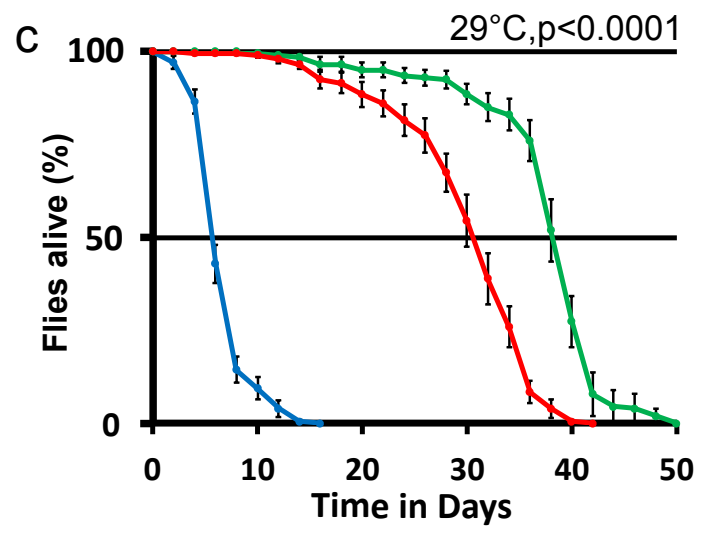

$\rightarrow$ hh mutant (repo-Gal4) $\rightarrow$ control (repo-Gal4)

$\rightarrow c i{ }^{a c t} O E$ (repo-Gal4) in $h h$ mutant

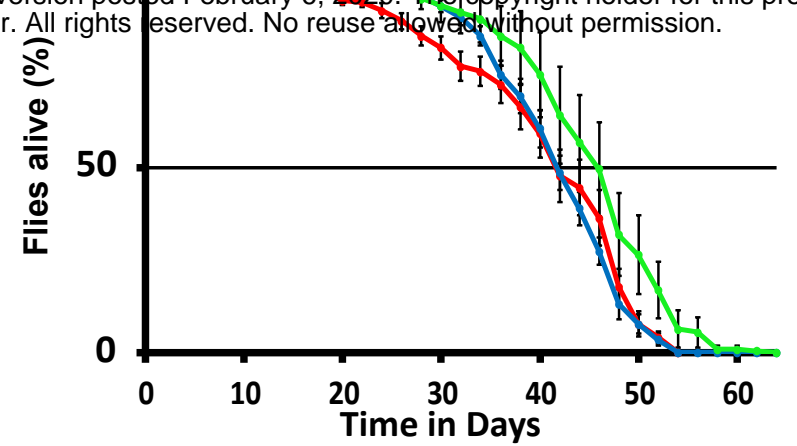

- RNAi smo (elav-Gal4) - control (elav-Gal4)

- RNAi ci (elav-Gal4)

d

- hh mutant (repo-Gal4)

- ci act $O E$ (repo-Gal4) in hh mutant

- control (repo-Gal4)
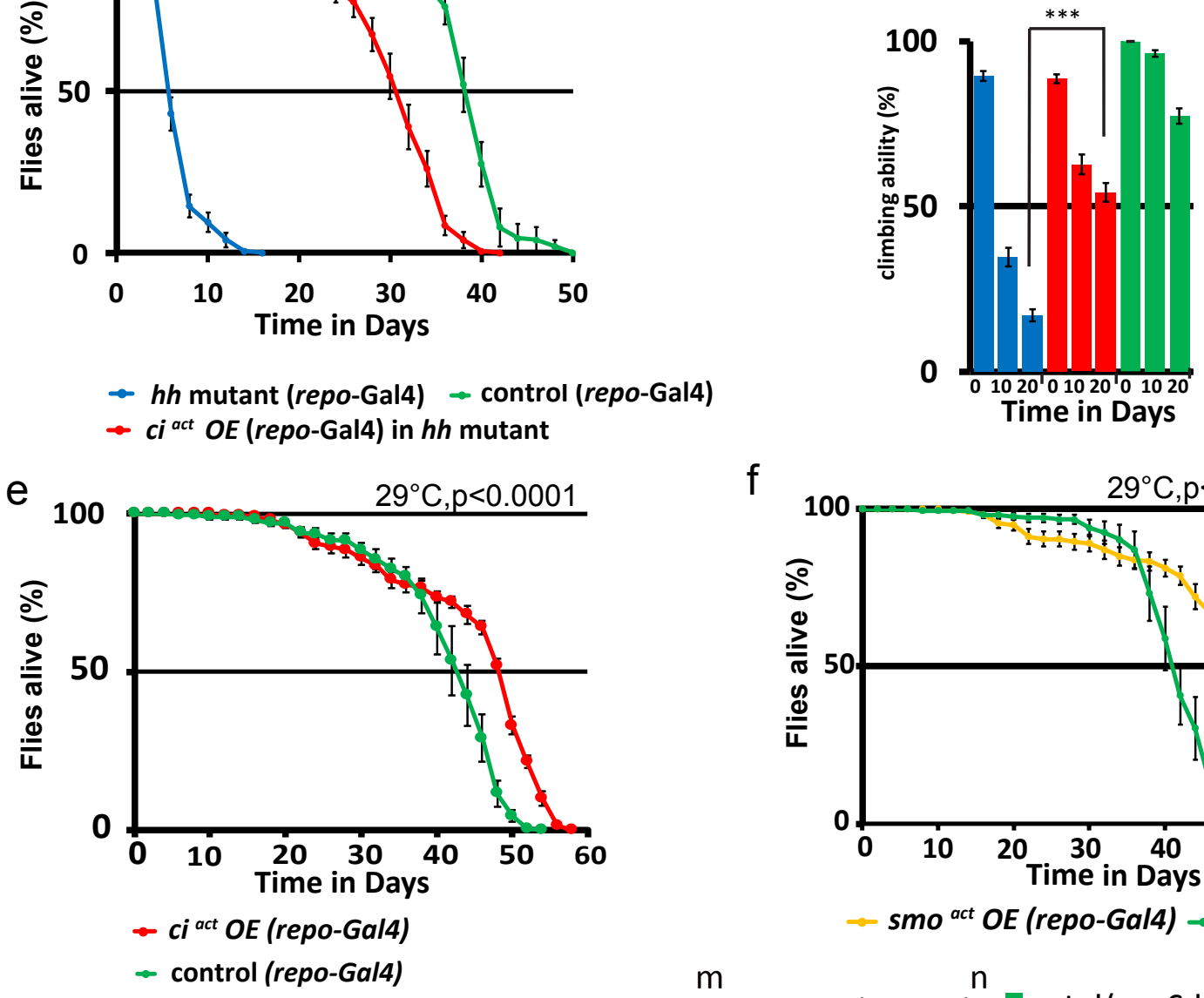

f

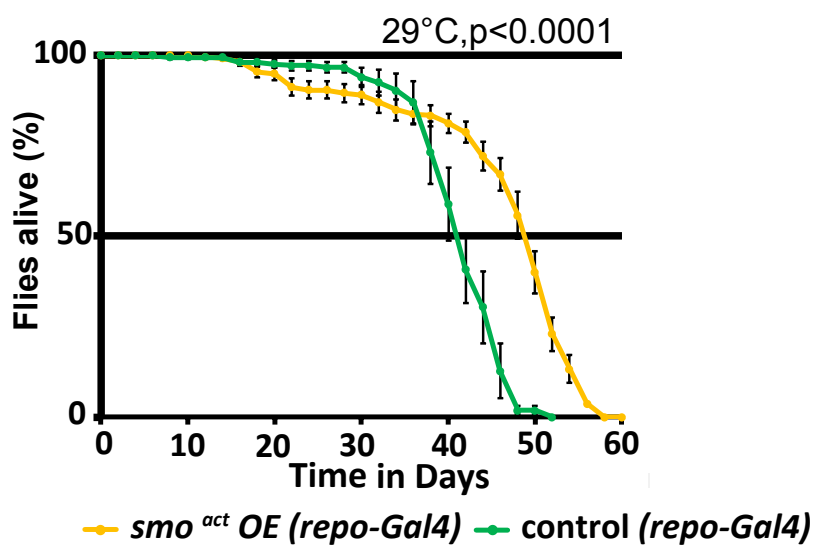

m

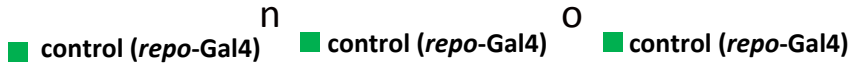 $\square h$ mutant (repo-Gal4) $\square h$ mutant (repo-Gal4) $\square h$ h mutant (repo-Gal4)
- ci $i^{\text {act }} O E$ (repo-Gal4) in $\square c c^{\text {act }} O E$ (repo-Gal4) in $\square c i^{\text {cct }} O E$ (repo-Gal4) in hh mutant hh mutant hh mutant
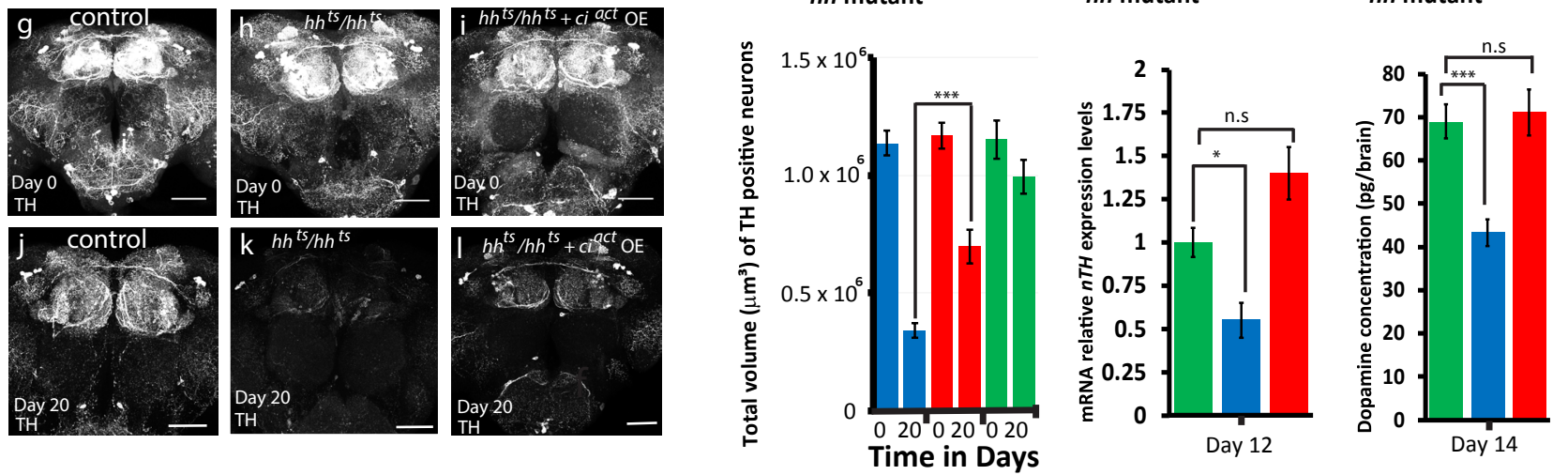


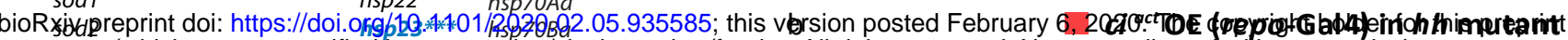

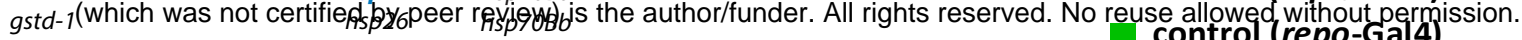

$\begin{array}{lll}\text { catalase } & h s p 27 & \text { hsc70-4 } \\ \text { Autophagy components } & \text { hsp40*** } & \text { hsp83 } \\ \text { lamp-1 } & h s p 67 B a & \text { BiP }\end{array}$

$\begin{array}{ll}\text { lamp-1 } & \text { hsp67Ba } \\ \text { atg } 8 a & \text { hsp68*** }\end{array}$

$\operatorname{atg} 1$
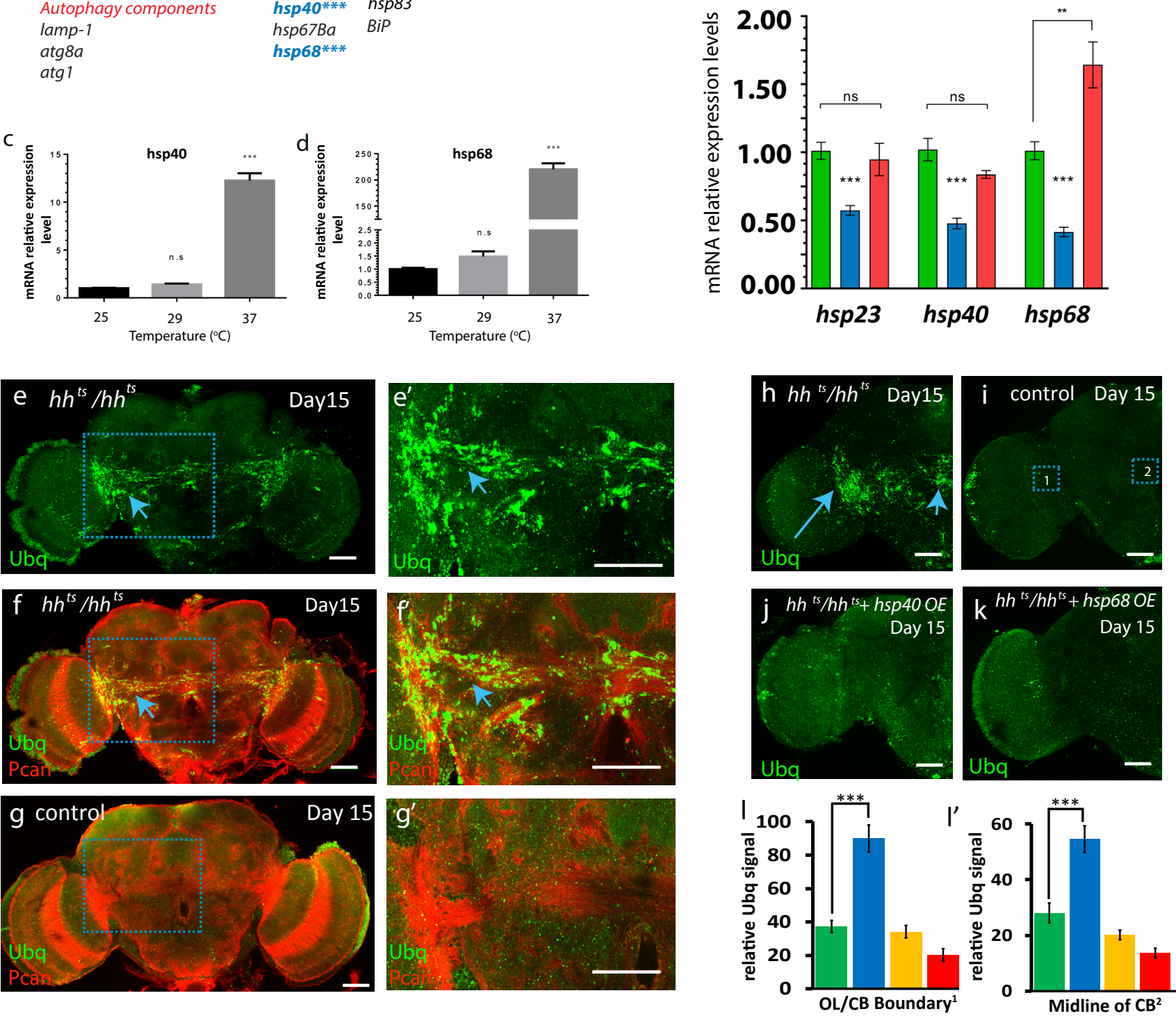

control (repo-Gal4)

hh mutant (repo-Gal4)
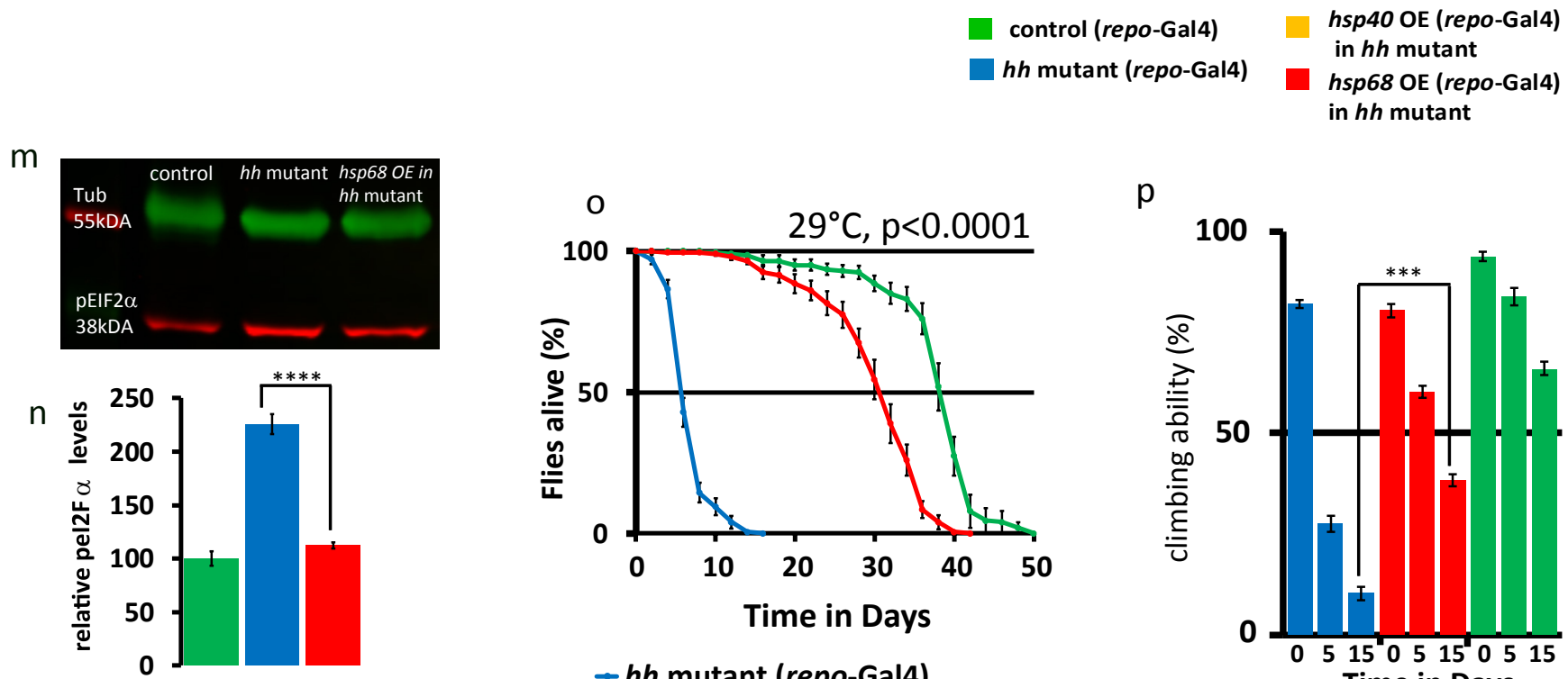

control (repo-Gal4)

- hh mutant (repo-Gal4)

hsp68 OE (repo-Gal4) in hh mutant
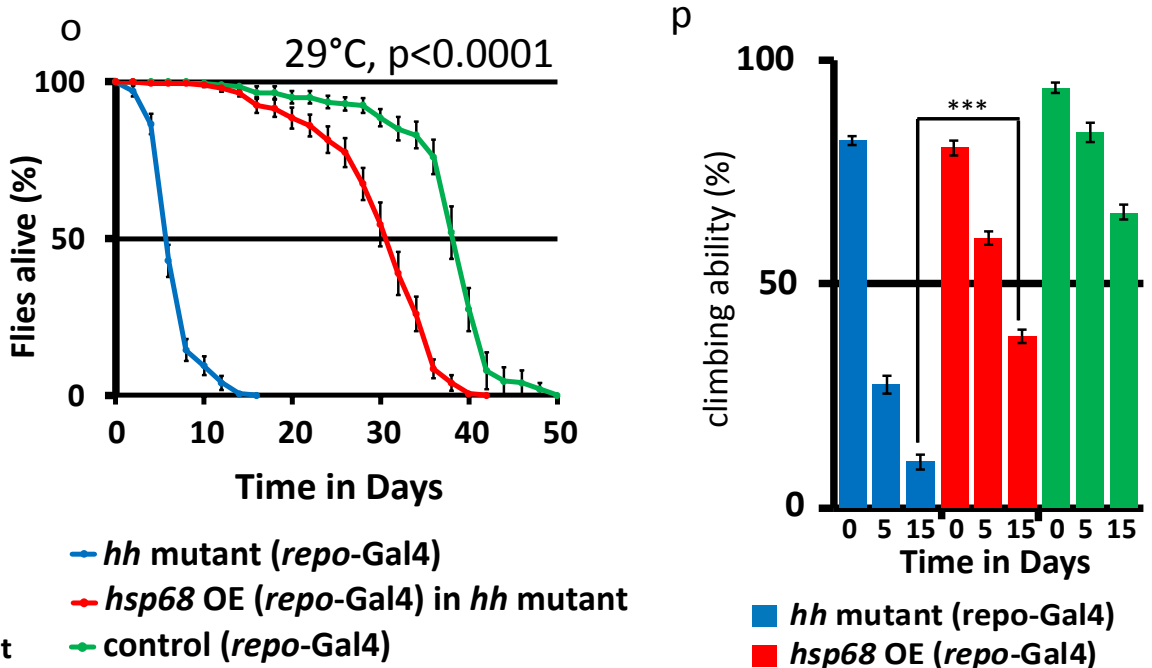

hh mutant (repo-Gal4)

- hsp68 OE (repo-Gal4)

in $h \boldsymbol{h}$ mutant

control (repo-Gal4) 


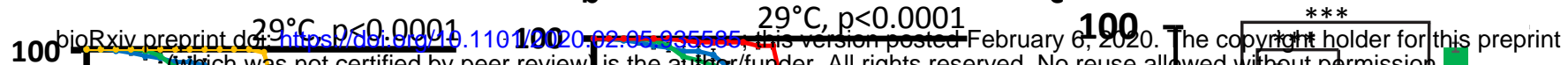

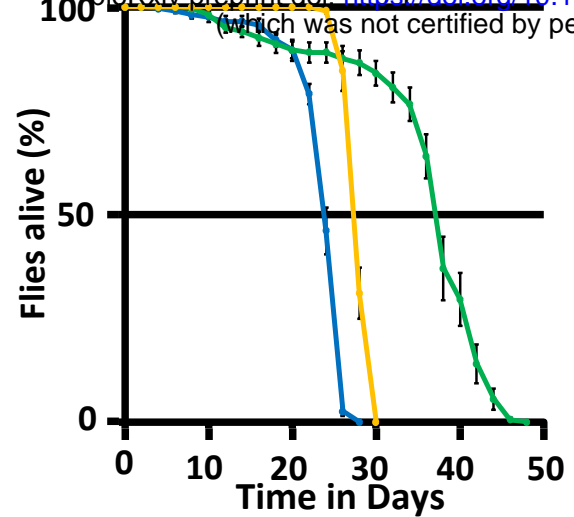

$-A \beta 42^{E 693 G}($ repo-Gal4) + GFP NLS

$-A \beta 42^{\mathrm{E} 693 \mathrm{G}}$ (repo-Gal4) + Smo ${ }^{\text {Act }}$

- control (repo-Gal4)

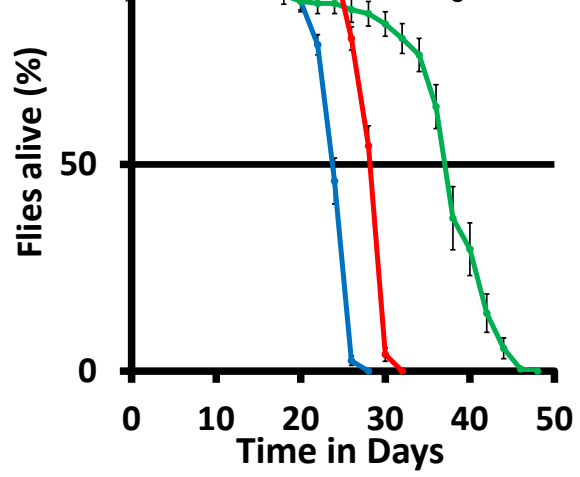

- A $\beta 42^{\text {E693G }}$ (repo-Gal4) + GFP NLS

- A $\beta 42^{\mathrm{E693G}}$ (repo-Gal4) + Hsp68

$\rightarrow$ control (repo-Gal4)

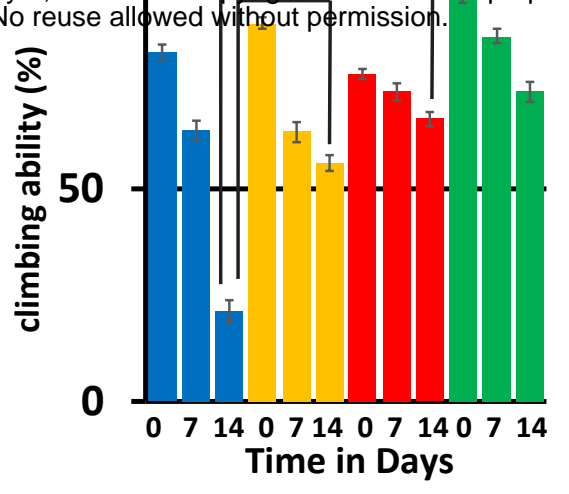

$A \beta 422^{\text {E693G }}$ (repo-Gal4) + GFP NLS

$A \beta 42^{\text {E693G }}$ (repo-Gal4) + Smo ${ }^{\text {Act }}$ $A \beta 42^{\text {E693G }}$ (repo-Gal4) + Hsp68 control (repo-Gal4)
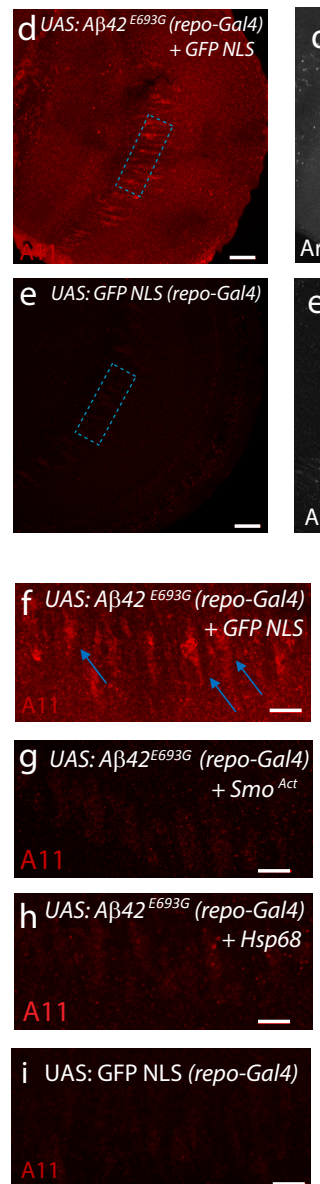
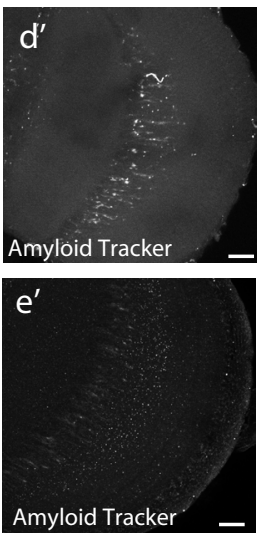
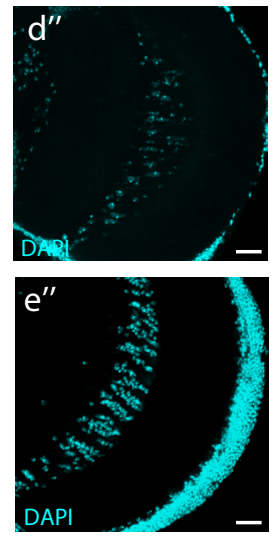
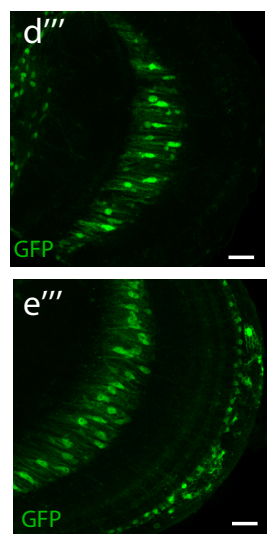
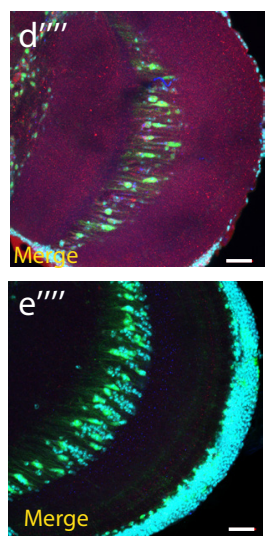
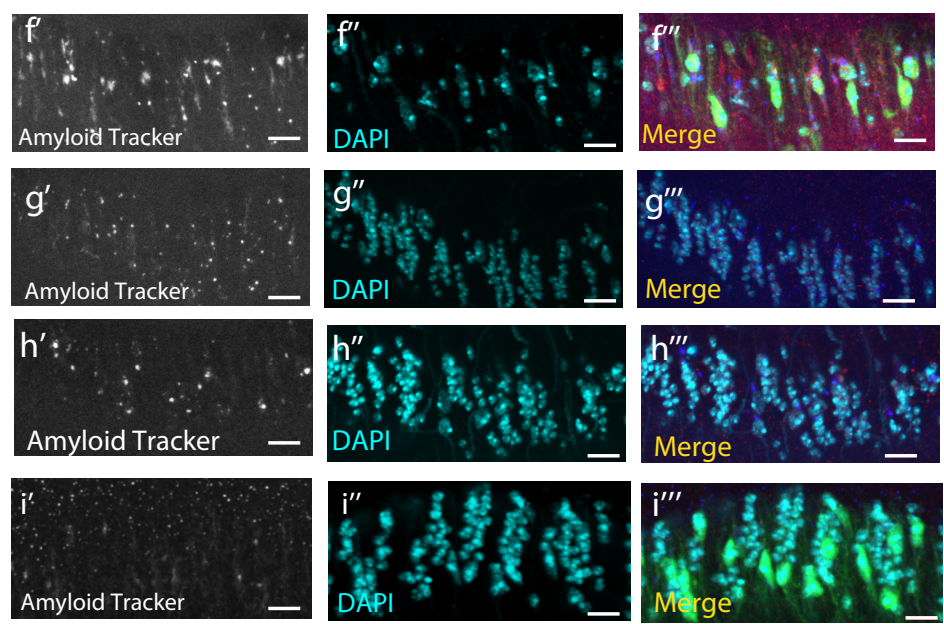

control (repo-Gal4)

A $\beta 42^{\mathrm{E} 693 \mathrm{G}}$ (repo-Gal4) + GFP NLS

A $\beta 42^{\mathrm{E} 693 \mathrm{G}}$ (repo-Gal4) + Smo ${ }^{\mathrm{Act}}$

- $A \beta 42^{\mathrm{E} 693 \mathrm{G}}$ (repo-Gal4) + Hsp68

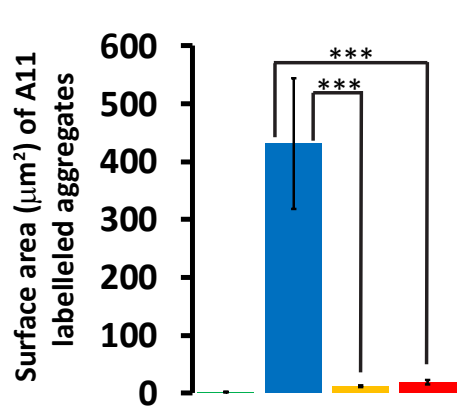

k

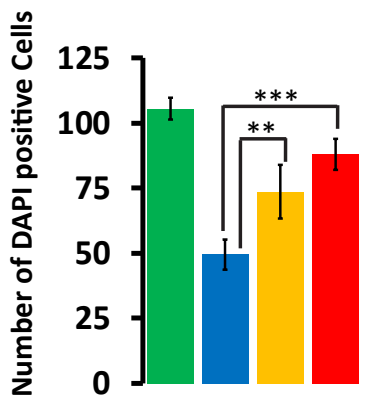


1. Activation of Hh signalling

2. Hh receiving module in glial cells

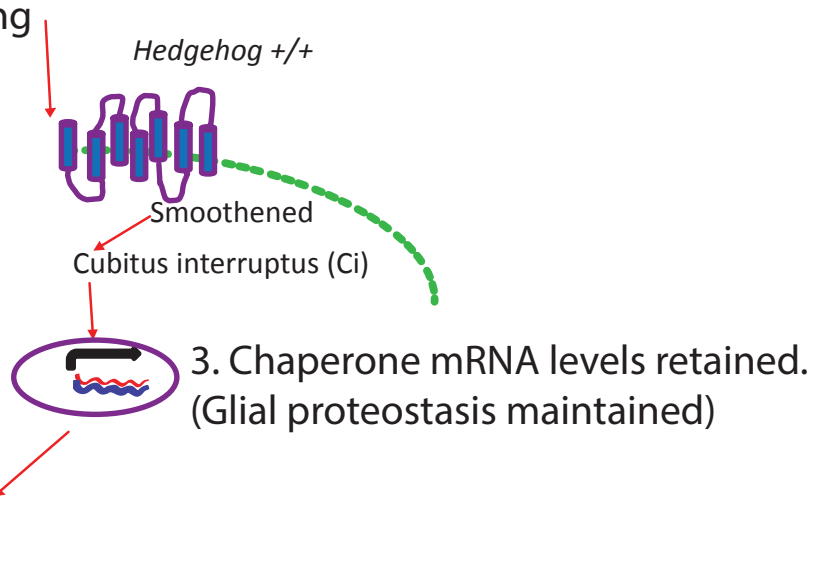

4. DA neuron integrity/Dopamine levels maintained

\section{Adult Brain in absence of Hh signalling - 'Hh signalling off'}

Hedgehog -/-

1. Inhibition of Hh signalling

2. Hh receiving module in glial cells

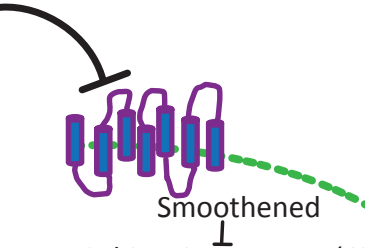

Cubitus interruptus (Ci)

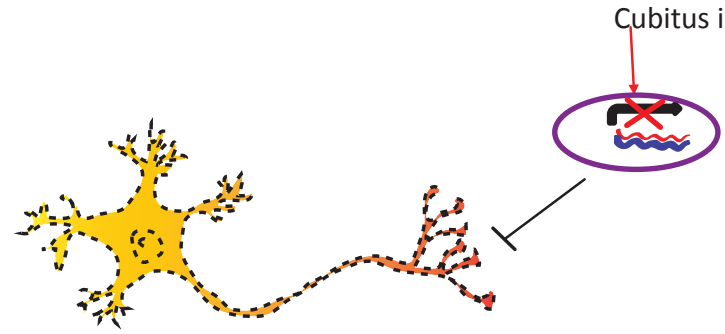

3. Chaperone mRNA levels decrease.

(Glial proteostasis declines).

\section{pEIF2A $\{$ Ubq $\uparrow$}

4. DA neuron degeneration/loss

lifespan\mobility】

\Dopamine \ Tyrosine Hydroxylase 

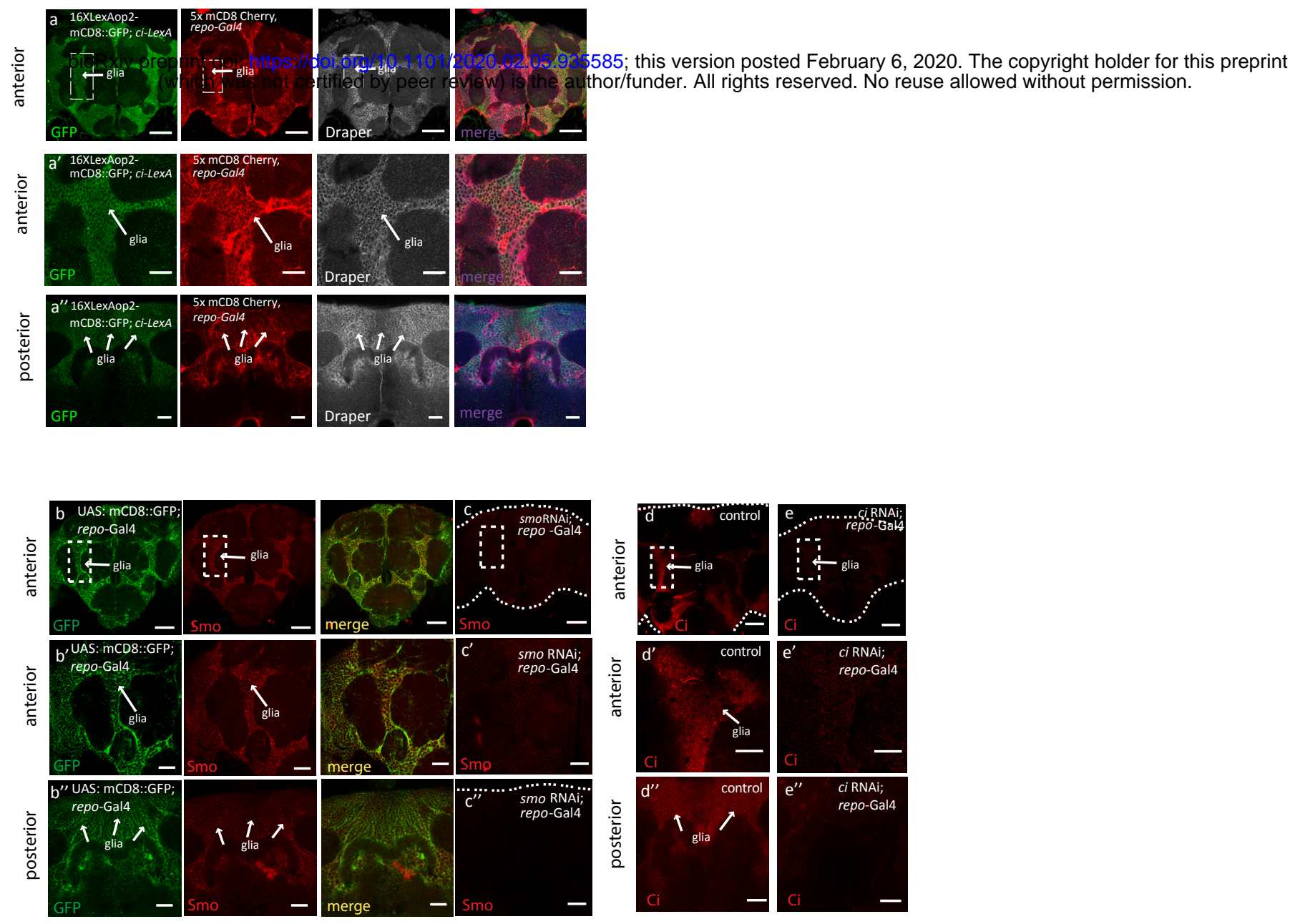

\section{f}

Schematic $h h$-gal4 trojan line
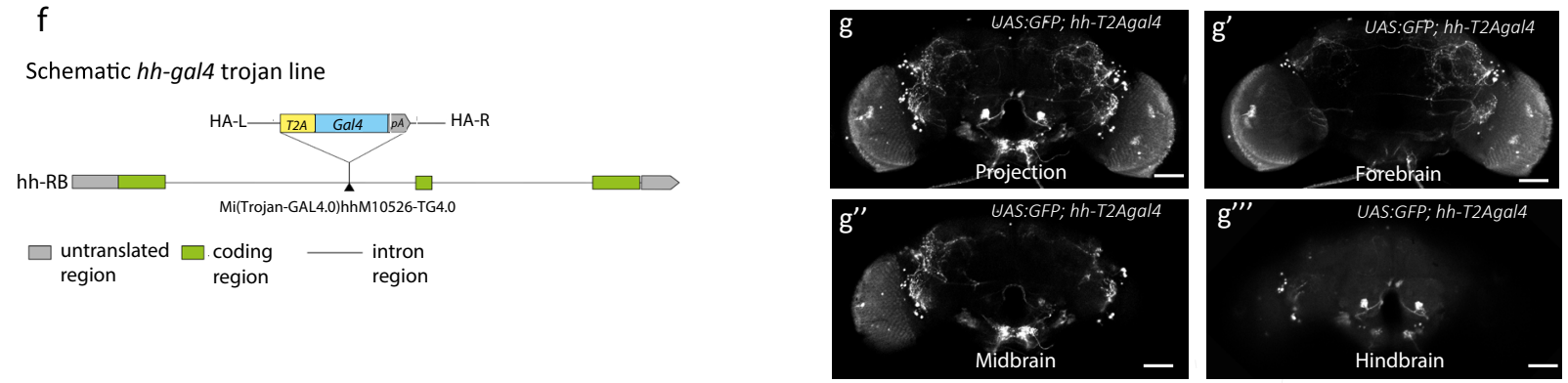

(h) $h$ h positive GABAergic neurons

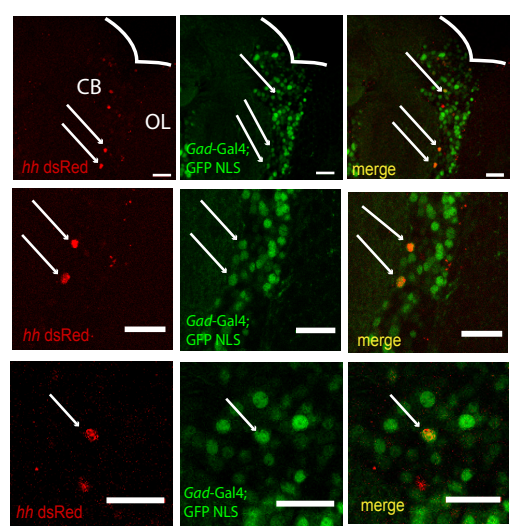

(i) $h h$ positive Glutamatergic neurons

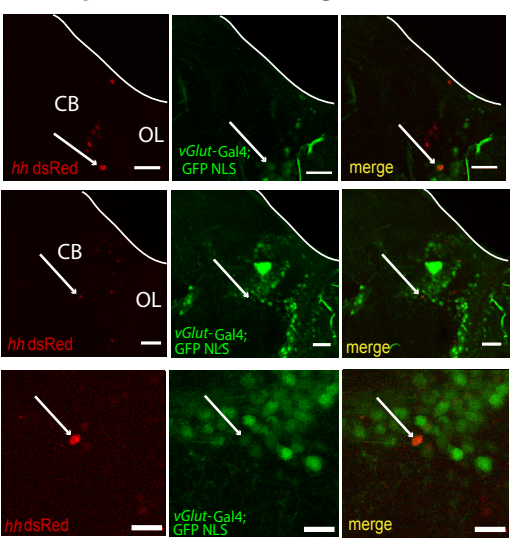

j

\begin{tabular}{|l|l|c|}
\hline $\begin{array}{l}\text { GAL4 } \\
\text { Driver }\end{array}$ & $\begin{array}{l}\text { Cell/Neuron } \\
\text { type }\end{array}$ & $h h$ dsRed \\
\hline Th-Gal4 & Dopaminergic & - \\
\hline ChAT-Gal4 & Cholinergic & - \\
\hline ddc-Gal4 & $\begin{array}{l}\text { Dopaminergic/ } \\
\text { Serotonergic }\end{array}$ & - \\
\hline vGlut-Gal4 & Glutamatergic & + \\
\hline Gad-Gal4 & GABAergic & + \\
\hline tdc2-Gal4 & Octopaminergic & - \\
\hline repo-Gal4 & Glia & - \\
\hline
\end{tabular}


bioRxiv preprint dd (which $\mathrm{n}$
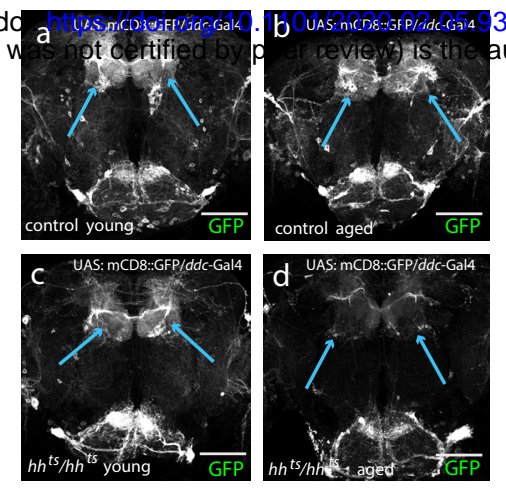

35585-this varsiont

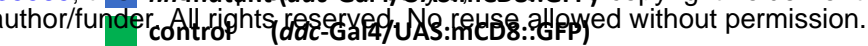
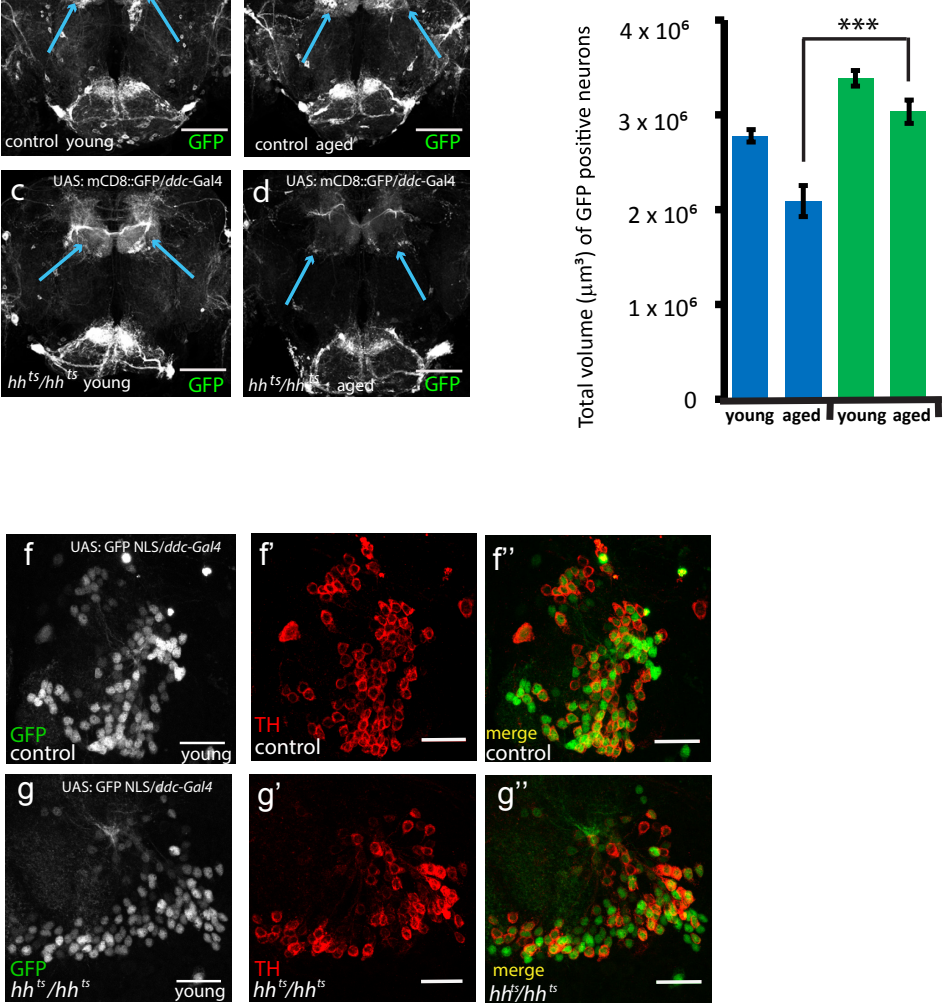

Figure S2. 
bioRxiv preprint doi: https://doi.org/1029
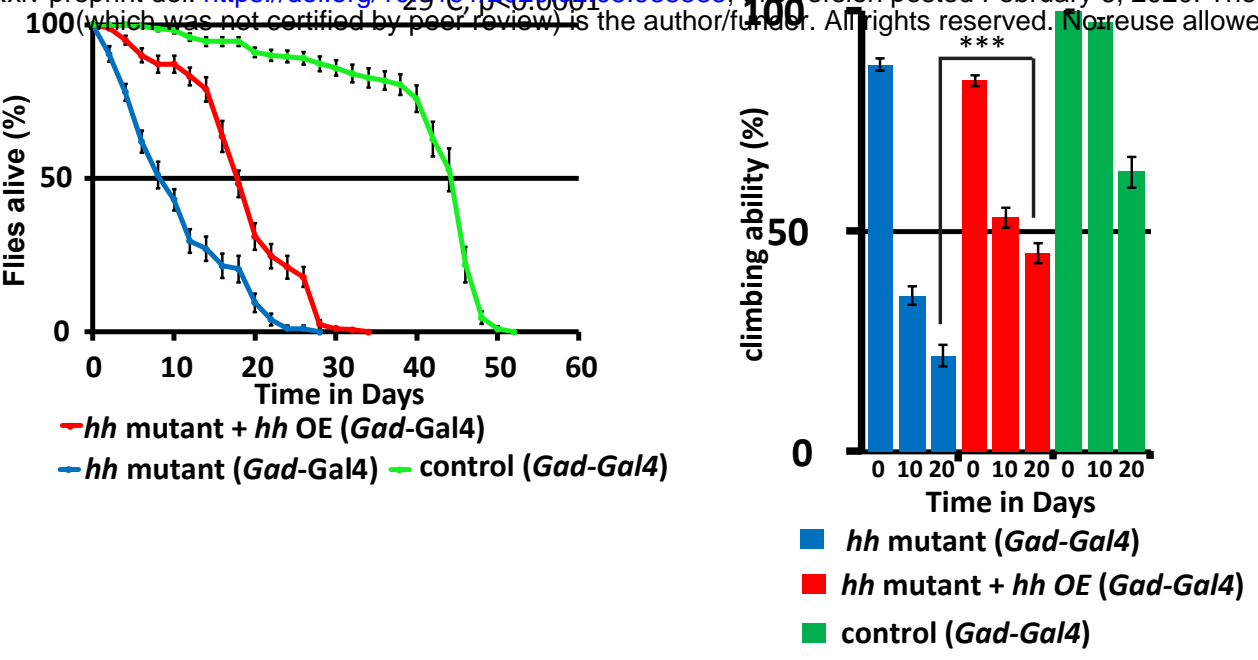

C

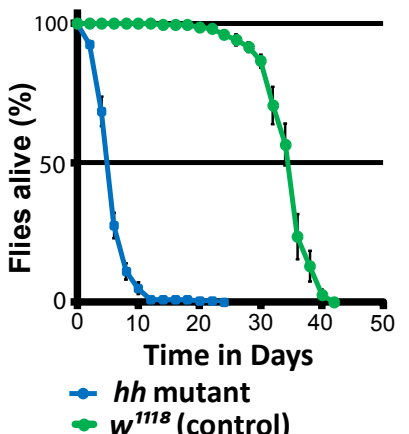

g

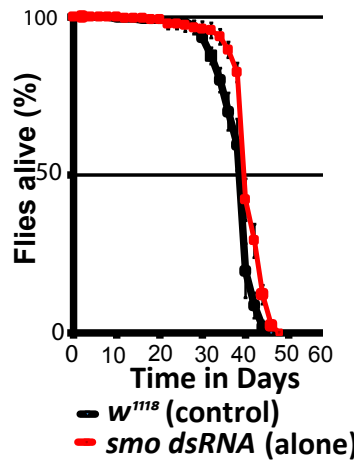

d

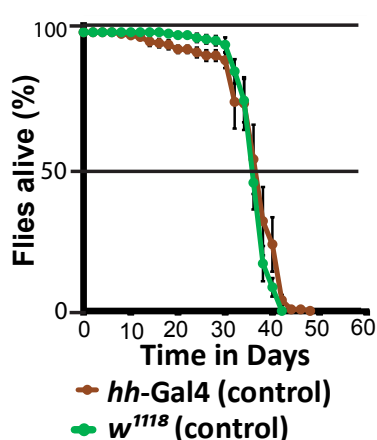

h

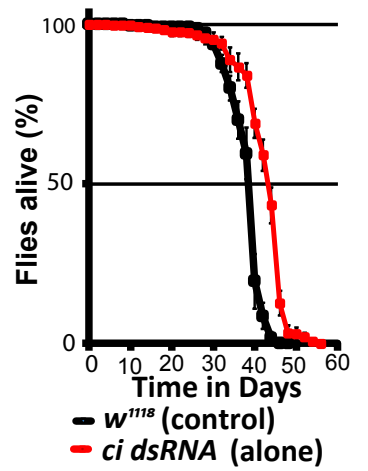

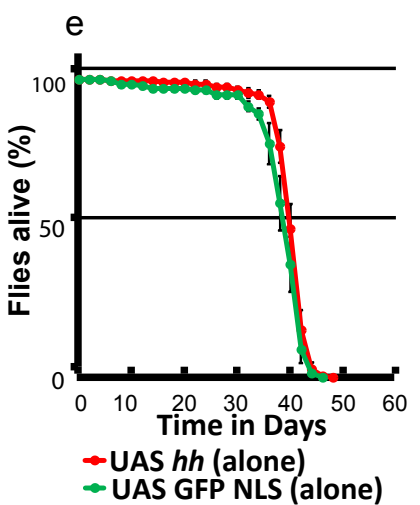
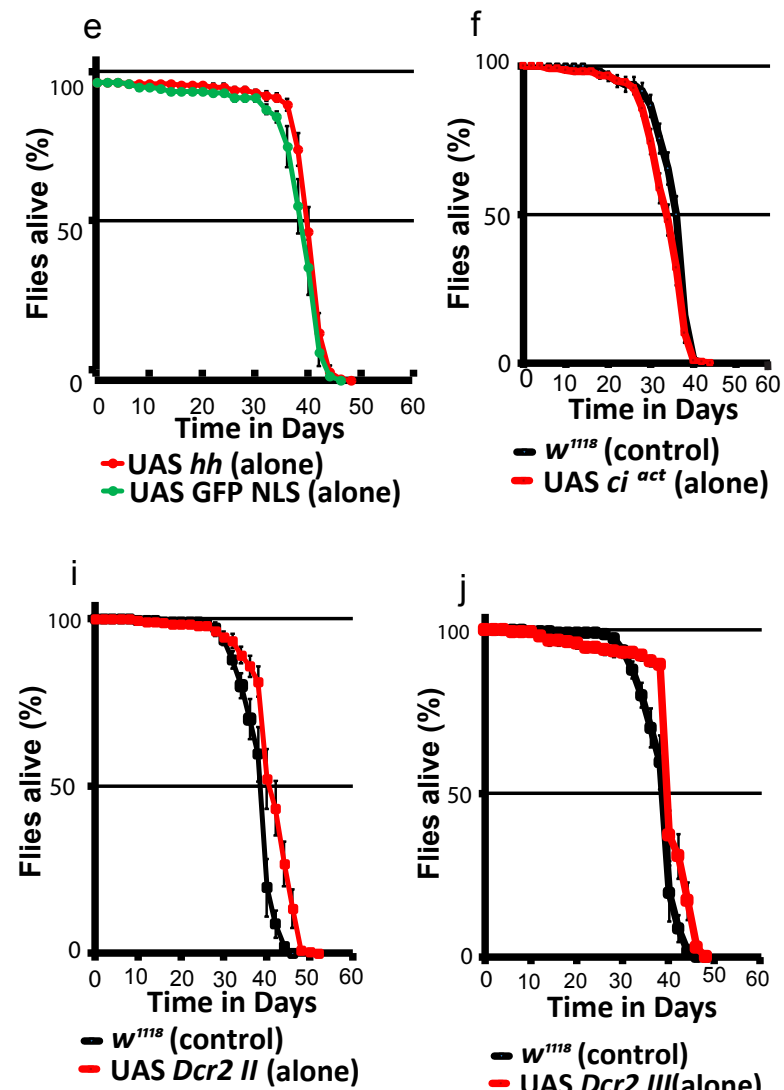

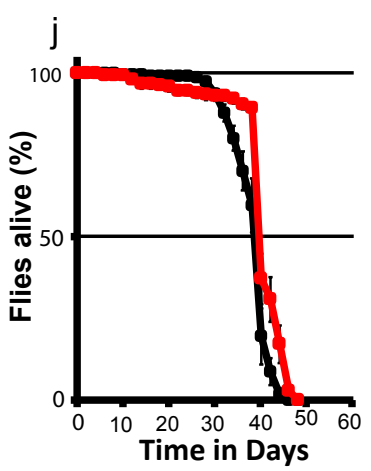
- UAS Dcr2 III(alone)
- $w^{m 118}$ (control)

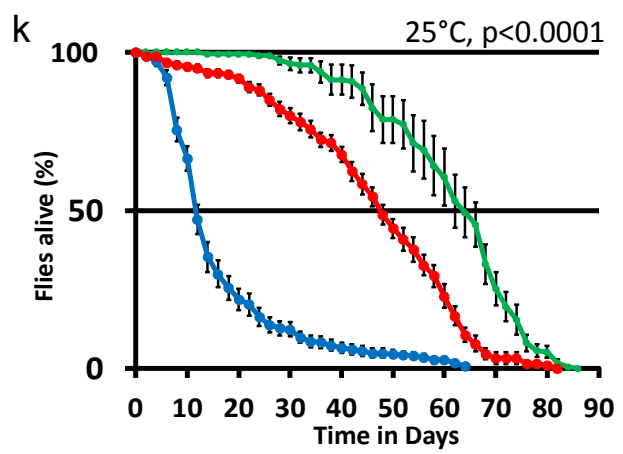

$\rightarrow$ hh mutant (repo-Gal4) $\rightarrow$ control (repo-Gal4)

- ci ${ }^{\text {act }}$ OE (repo-Gal4) in hh mutant 


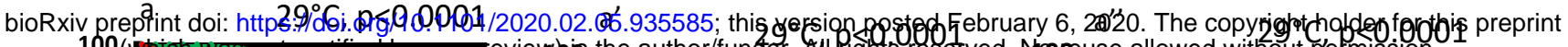
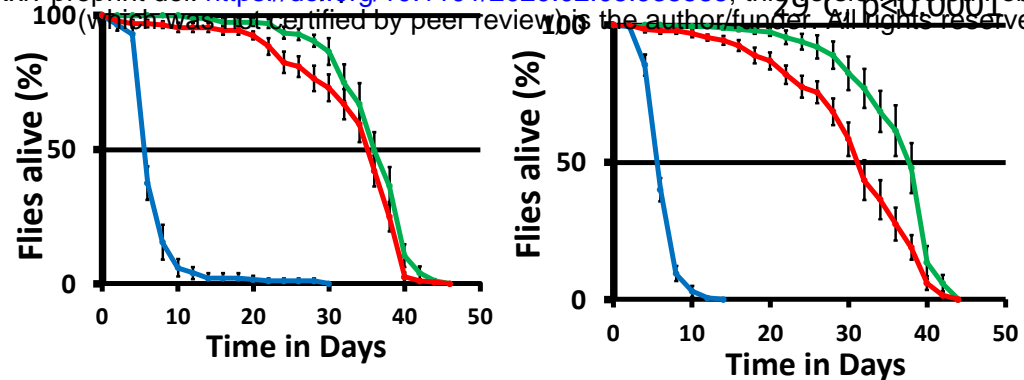

- hh mutant (repo-Gal4)

$\rightarrow$ ci $^{\text {act }} \mathrm{OE}($ repo-Gal4) in $h$ h mutant

$\rightarrow$ control (repo-Gal4)

\section{- hh mutant (repo-Gal4)}

$\rightarrow c i^{\text {act }} \mathrm{OE}($ (repo-Gal4) in $h$ h mutant

$\rightarrow$ control (repo-Gal4)

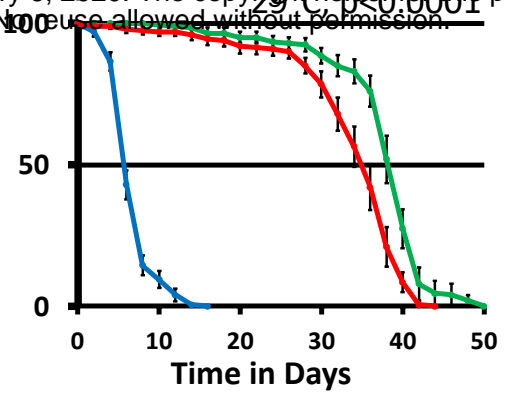

- hh mutant (repo-Gal4)

- ci ${ }^{\text {act }}$ OE (repo-Gal4) in hh mutant

- control (repo-Gal4)
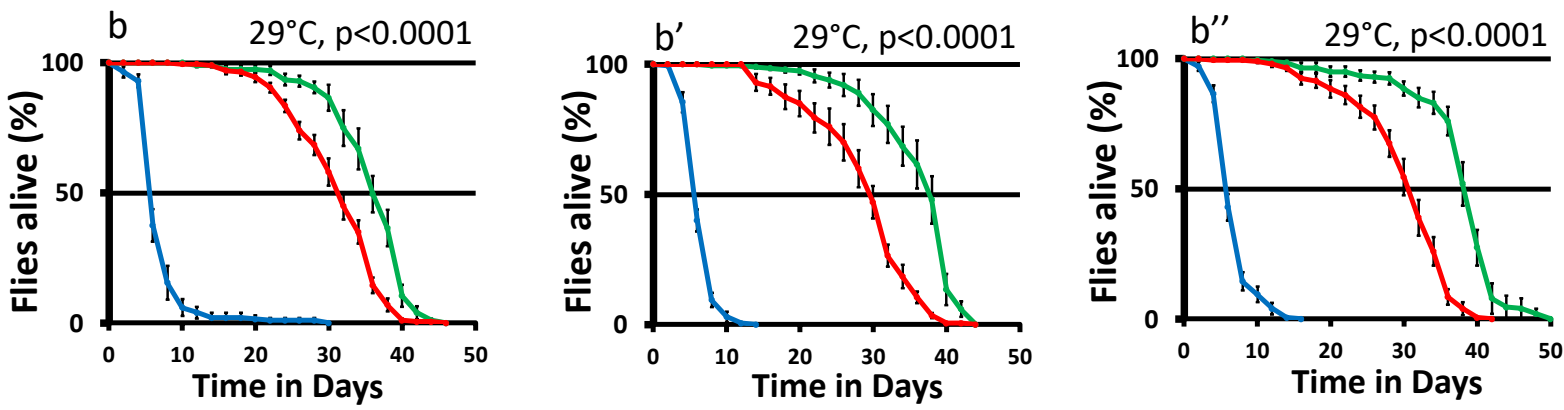

$-h h$ mutant (repo-Gal4)

- hsp68 OE (repo-Gal4) in hh mutant

$\rightarrow$ control (repo-Gal4)

- hh mutant (repo-Gal4)

- hsp68 OE (repo-Gal4) in hh mutant

- hh mutant (repo-Gal4)

- control (repo-Gal4)

$\rightarrow$ control (repo-Gal4)
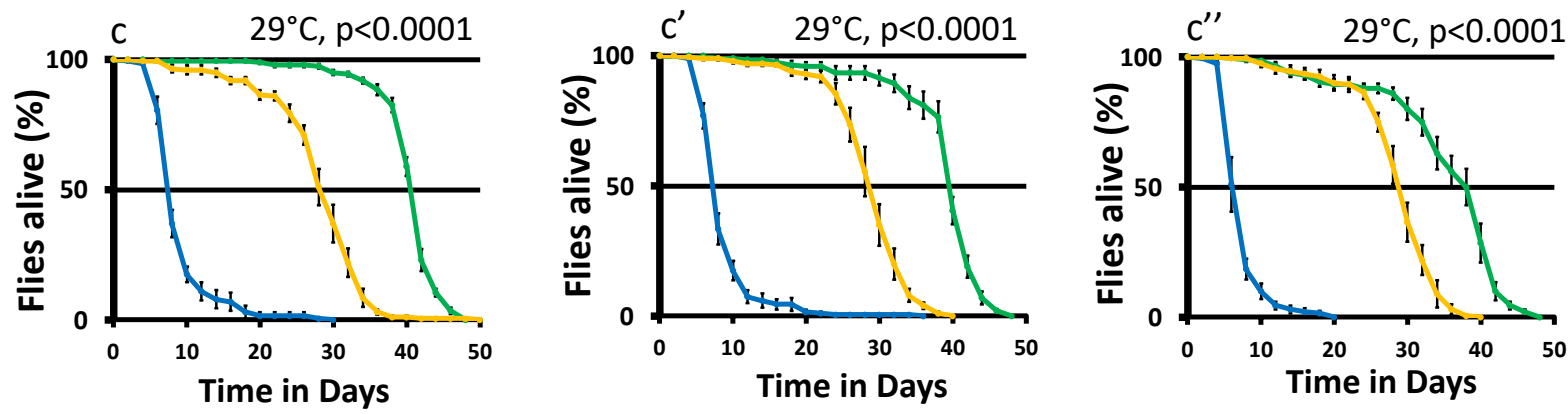

- hh mutant (repo-Gal4)

$\rightarrow h h$ mutant (repo-Gal4)

- hh mutant (repo-Gal4)

- hsp40 OE (repo-Gal4) in hh mutant

$-h s p 40$ OE (repo-Gal4) in hh mutant

- control (repo-Gal4)

- control (repo-Gal4)
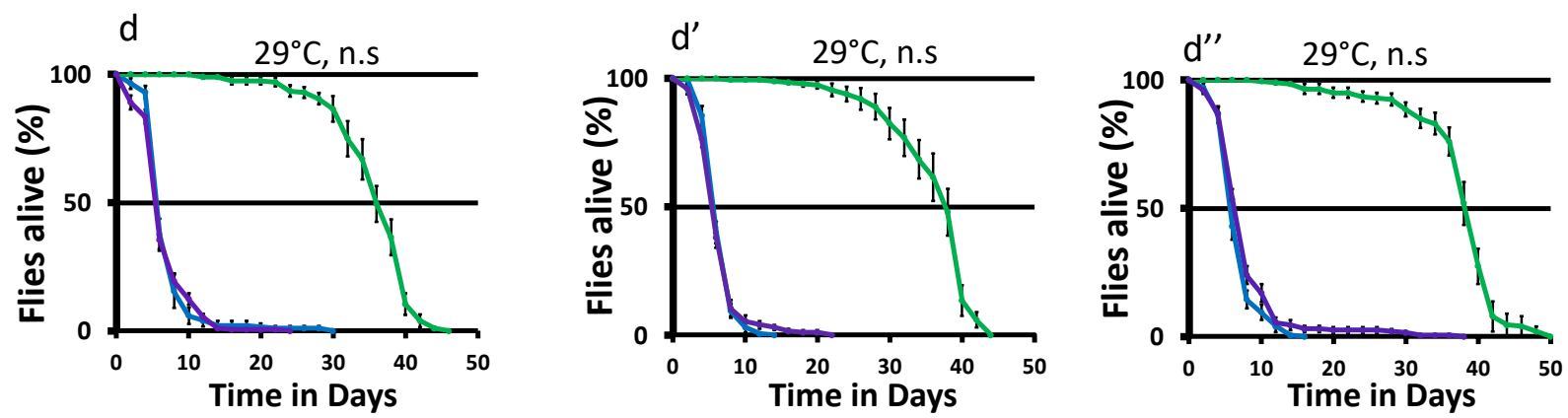

- hh mutant (repo-Gal4)

- hsp23 OE (repo-Gal4) in hh mutant - control (repo-Gal4)

- hh mutant (repo-Gal4)

- hsp23 OE (repo-Gal4) in hh mutant

- control (repo-Gal4)

- hh mutant (repo-Gal4)

- hsp23 OE (repo-Gal4) in hh mutant

- control (repo-Gal4) 

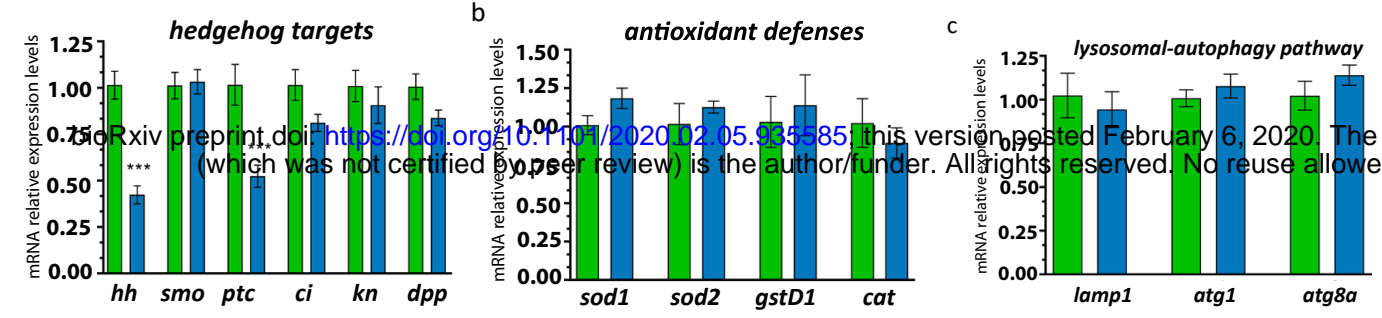

c
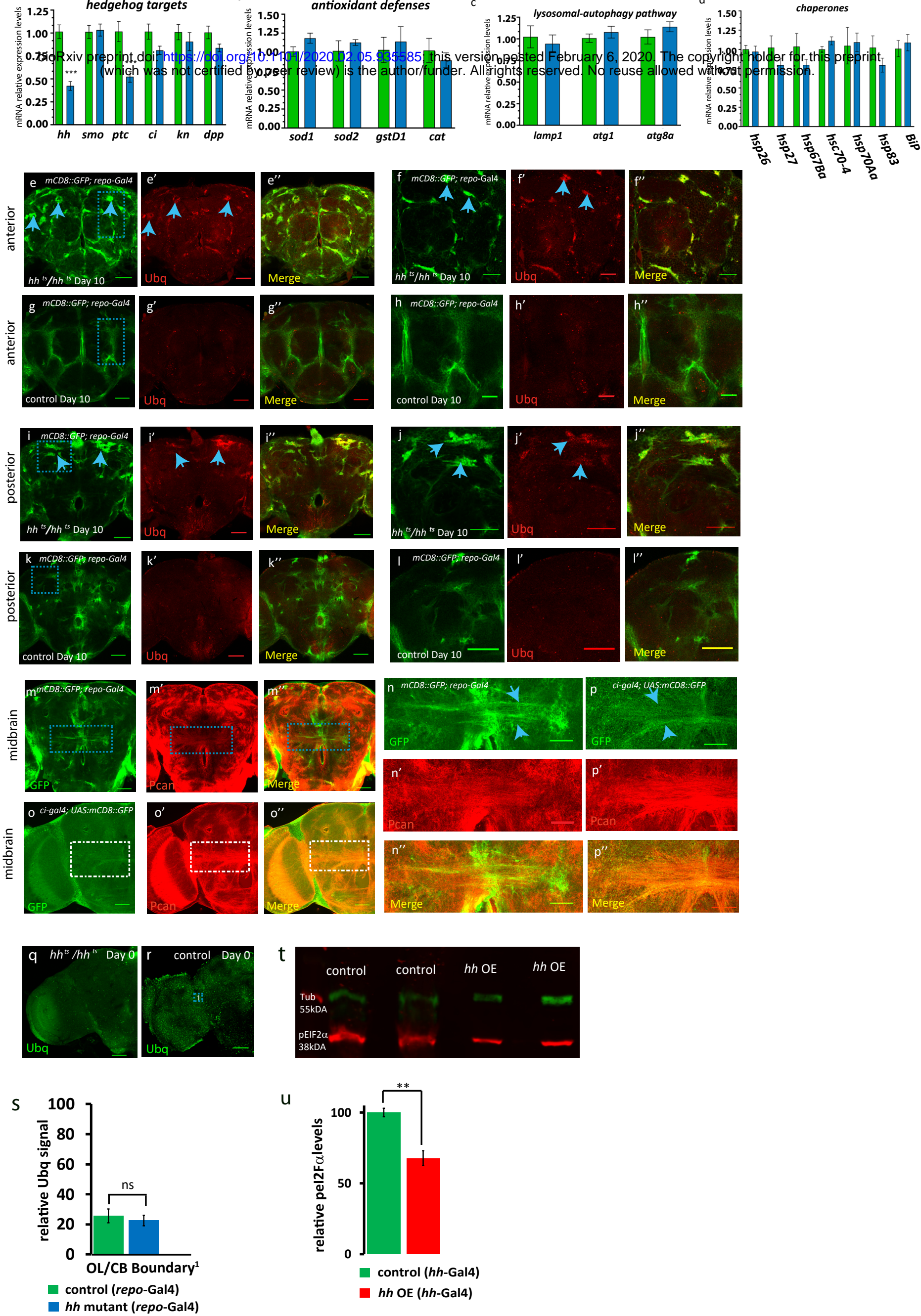

Figure S5. 

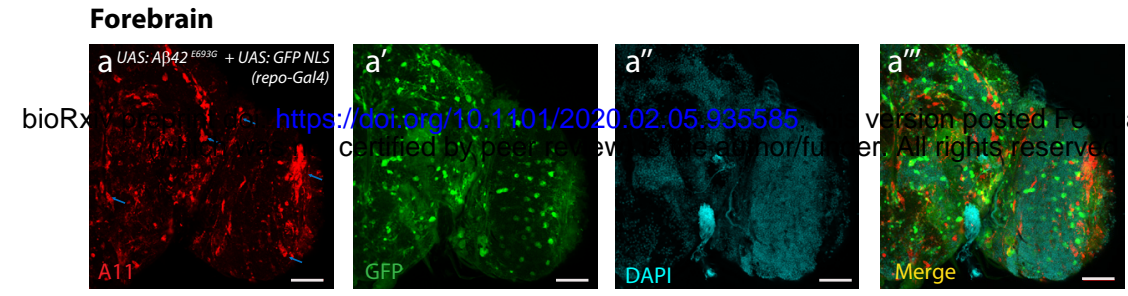

ary 6,2020 . The copyright holder for this preprint No reuse allowed without permission.
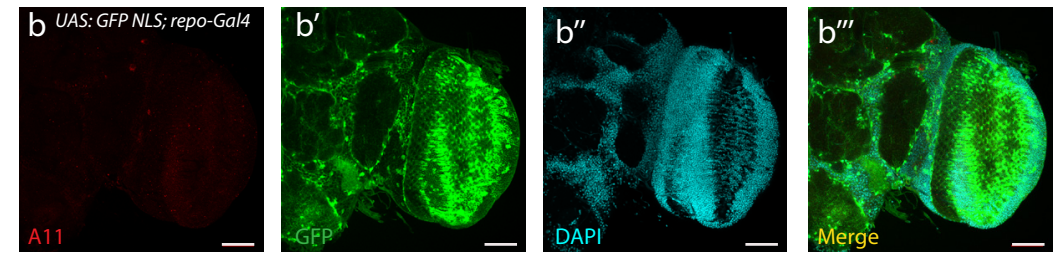

Hindbrain
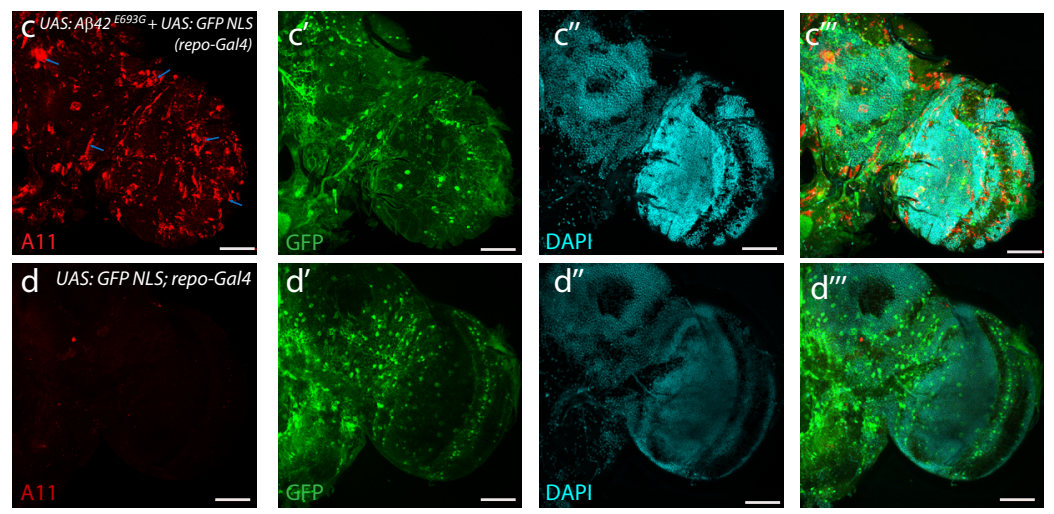

Midbrain
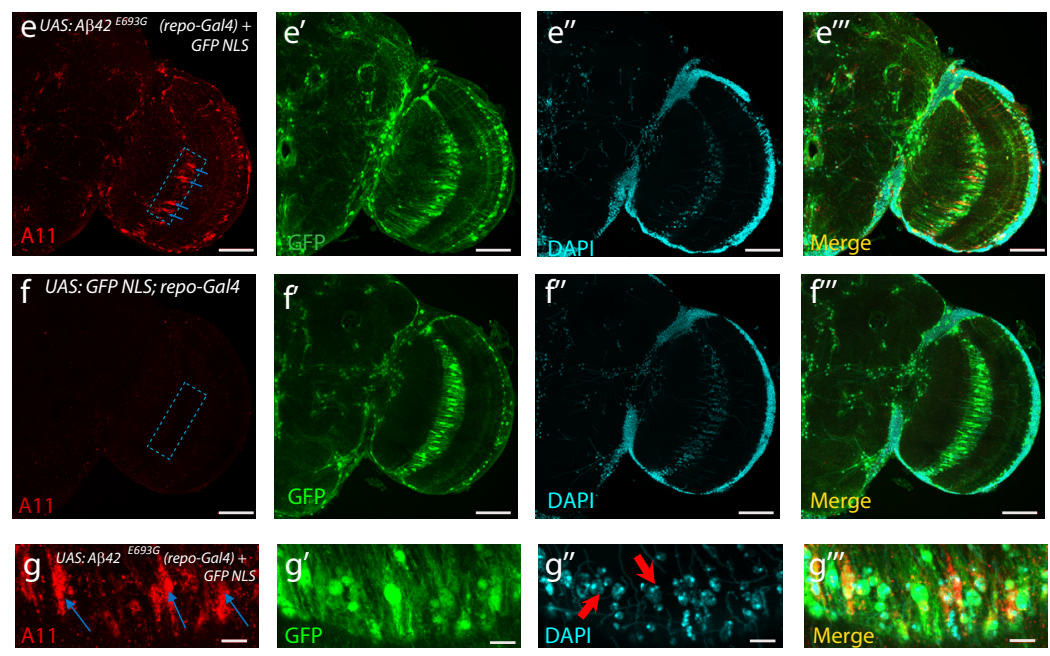

$\mathrm{h}$
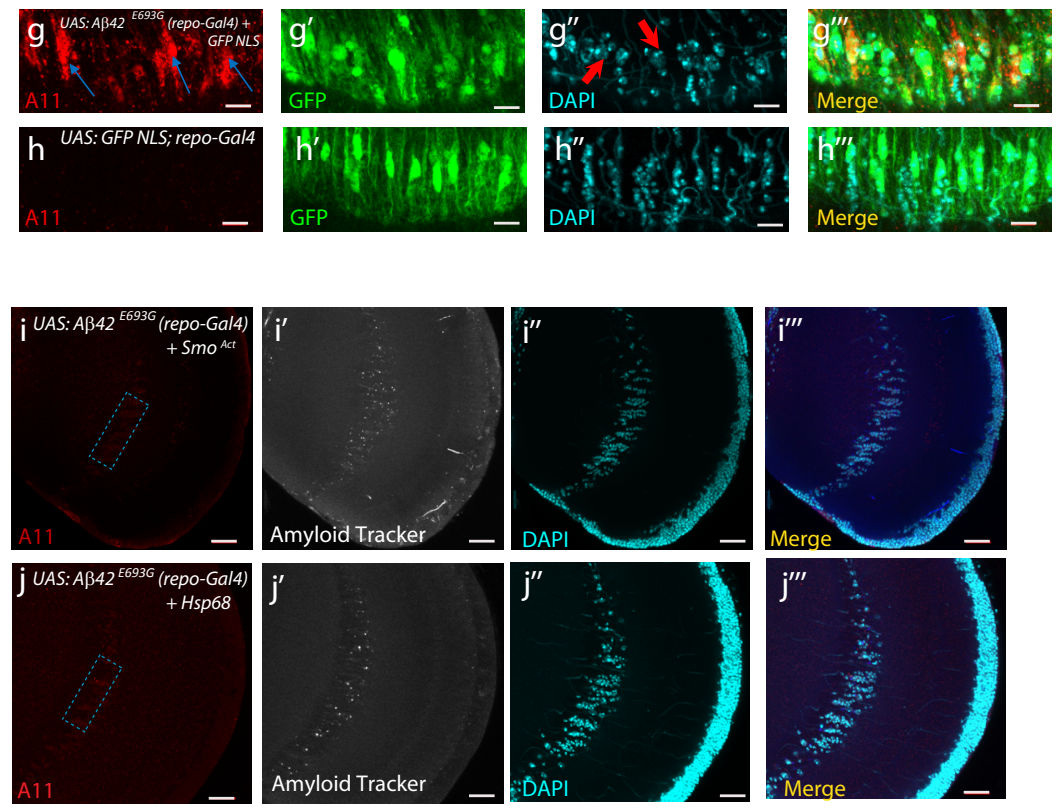

Figure S6.

Rallis et al, 2019 


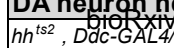

ing 505 ;

(3) 480914 .

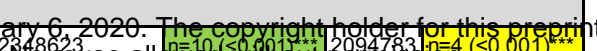

control: Ddc-GAL4/UAS:mCD8::GF

DA neuron network volume of Hh mutants (TH immunostaining)

\begin{tabular}{l|l}
$h h^{\text {ts2 }}, D d c-G A L 4 /$ UAS:mCD8::GFP, $h h^{\text {ts2 }}$ & $1 \mathrm{~m}$,
\end{tabular}

control: Ddc-GAL4/UAS:mCD8::GFP

$1 \mathrm{k}, 1 \mathrm{l}$

1323223 n=12 (n.s)

1097554.8

\begin{tabular}{|l|l|l|}
\hline$n=10(<0.05)^{\star}$ & 651896 & $n=4(<0.001)^{\star * *}$ \\
\hline$n=10(<0.05)^{\star}$ & 1178530 & $n=8(<0.001)^{* * *}$
\end{tabular}

Genotype

Figure $1331695 \quad n=10$ (n.s)

1272523.2

$\mathrm{n}=10(<0.05)^{\star}$

DA neuron volume of Hh mutant flies expressing $\mathrm{Ci}$ Act In glia (TH immunostaining)

control: UAS:GFP NLS/GAL80 ${ }^{\text {ts }}$;Repo-GAL4/+

GAL80 $^{\text {ts }} /+;$ UAS: GFP NLS, $h h^{\text {ts } 2} /$ Repo-GAL4, $h h^{\text {ts } 2}$

$3 g, 3 j$

GAL80 ${ }^{t s} /+;$ UAS:Ci PKA, $h h^{\text {ts } 2} /$ Repo-GAL4, $h h^{\text {ts } 2}$

$3 \mathrm{~h}$

\begin{tabular}{l|l|l}
\hline $3,3 k$ & 1135597 & $n=10$ \\
\hline
\end{tabular}

\begin{tabular}{l|l|l}
$3 \mathrm{i}, 3 \mathrm{l}$ & 1166615 & (n.s) $\mathrm{n}=10$ \\
\hline
\end{tabular}

995048.5

P value

\begin{tabular}{l|l} 
& 341226.125 \\
\hline
\end{tabular}

$\mathrm{n}=6(<0.001)^{\star * *}$

$\mathrm{n}=5(<0.001)^{\star * *}$ 


\section{Supplementary Table 2: Summary of Lifespan Assays}

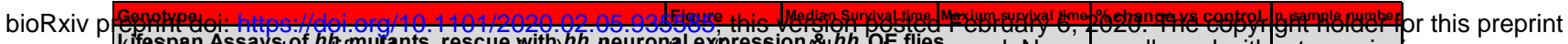

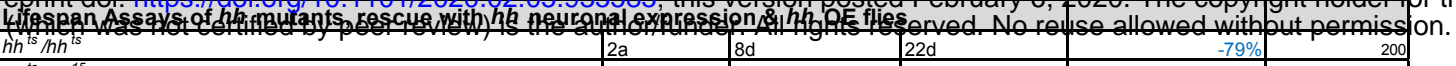

control: $w^{111}$

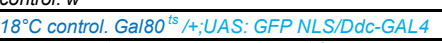

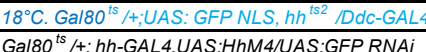

control: Gal80ts/t; ;hh GAL4/UAS:GFP RNAi

$\mathrm{h}^{\mathrm{ts}} \mathrm{hh} h^{\mathrm{t}}$

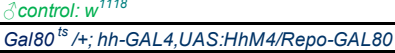

Gal80 is $t+$; hh-GAL4,UAS:HhM4/Elav-GAL80:

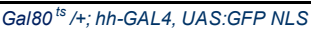

Gal80 ts + ; $;$ hh-GAL4,UAS:HhM4/UAS:GFP NLS

control: UAS:GFP NLS/Gal80 $0^{\text {ts }}$ :VGlut-GAL4

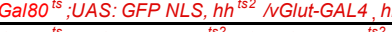

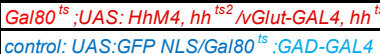

Gal80 $0^{15} ;$ UAS: GFP NLS, $h h^{152} /$ GAD-GAL4, hh

Gal80; :UAS

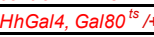

control: UAS:GFP $N L S /+$

Lifespan Assays of Ci/Smo RNAi knockdown and $h h$ mutant rescue with $\mathrm{C}^{\text {act }}$ in $\mathrm{G}$

control: UAS: Dcr2/Gal80 ts; UAS:GFP RNAi/Repo-GAL4

UAS: Smo RNAi/+; Smo ${ }^{\times 43}$, UAS: Dcr2/Gal80 $0^{\text {ts }} ;$ Repo-GAL4/+

UAS: Ci RNAi/Gal80 $0^{\text {ts }} ;$ UAS:Dcri/Repo-Gal4

control: ELAV-GAL4/+:UAS: DCr2/Gal80 $0^{\text {s: }}$ UAS:GFR RNAi/

UAS: Smo RNAilELAV-GAL4;Smo ${ }^{443}, U A S: D c r 2 / G a / 80^{t s}$;

ELAV-GAL4/+;UAS:Dcr2/Gal80 $0^{\text {ts }} ;$ UAS:Ci RNAi/ +

control: UAS:GFP NLS/Gal $80^{\text {ts }}$;Repo-GAL4/+

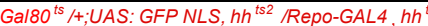

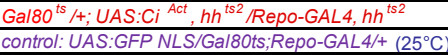

Gal80 is $t+;$ UAS: GFP NLS, hhts2 /Repo-GAL4, hits2 $\left(25^{\circ} \mathrm{C}\right.$

Gal $80^{15}+$ + UAS:Ci ${ }^{\text {Act }}$ hhts $2 /$ Repo-GAL4, hits2 $2\left(25^{\circ} \mathrm{C}\right.$

Exp1.control: UAS:GFP NLS/Gal/80 $0^{\text {ts }}$; Repo-GAL4/+

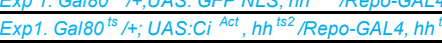

Exp2. control: UAS:GFP NLS/Gal80 ts ;Repo-GAL4/+

Exp2. Gal80 $0^{\text {ts }} /+;$ UAS: GFP NLS, $h h^{\text {ts2 }} /$ Repo-GAL4, $h h^{\text {ts2 }}$

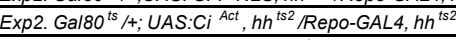

Exp3. Gal80 is $1+;$ UAS: GFP NLS, $h h^{\text {ts2 }} /$ Repo-GAL4, hh

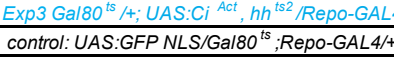

Gal80 ${ }^{\text {ts }} /+$ : UAS:Ci ${ }^{\text {Act }} /$ Repo-GAL4

control: UAS:GFP NLS/Gal80ts:Repo-GAL4

Gal80ts/+; UAS:Smo ${ }^{\text {Act } / R e p o-G A L 4}$

Exp1. control: w1118

UAS: $\mathrm{Ci}^{\mathrm{Act} / \mathrm{t}}$

Exp2. control: W1118

UAS/SMO RNAi/+

UAS:CIRNAII/

UAS:Dcr2/+ (III) -
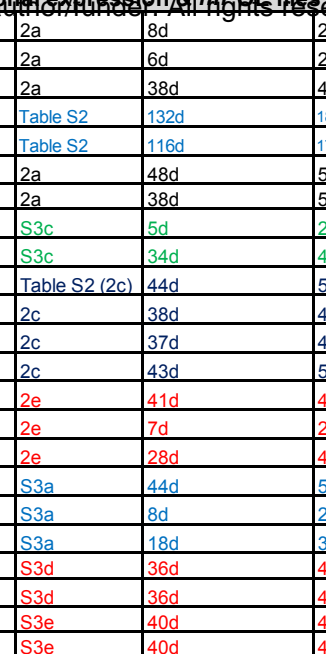

Lifespan Assays of $h h$ mutant rescue with expression of $H s p 23, H s p 40$ and $H s p 68$ in the Glia

Exp1.control: UAS:GFP NLS/Gal80 $0^{\text {ts }}$; Repo-GAL4/A

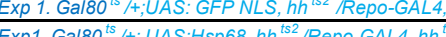

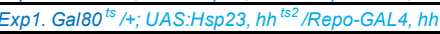

Exp2. control: UAS:GFP NLS/Gal80 ts; Repo-GAL4/+

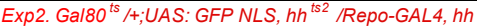

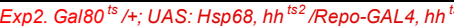

Exp2. Gal $80^{t s} /+;$ UAS: Hsp 23, $h h^{\text {ts2 }} /$ Repo-GAL4, $h h^{\text {ti2 }}$

Exp3. control: UAS:GFP NLS/Gal818 ; Repo-GAL4/+

Exp3. Gal80 $1 / 4+, U A S:$ GFP NLS, hht

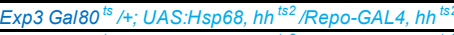

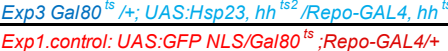

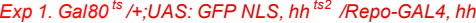

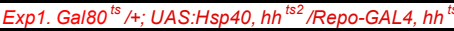

Exp2. control: UAS:GFP NLS/Gal80 ${ }^{15}$;Repo-GAL4/+

Exp2. Gal80 ts $/+;$ UAS: GFP NLS, $h h^{\text {ts2 }} /$ Repo-GAL4, hh

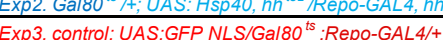

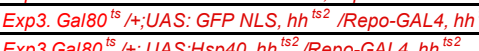

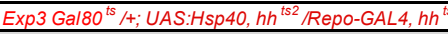

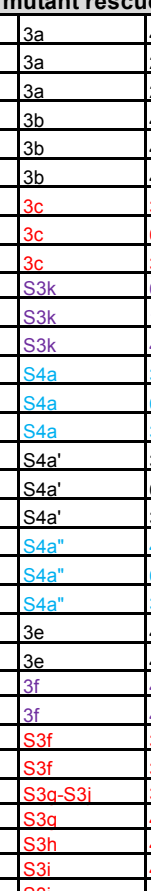

$44 d$

$25 \mathrm{~d}$

$23 \mathrm{~d}$

$42 \mathrm{~d}$

$42 \mathrm{~d}$

$+2$

)

6d

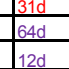

$38 \mathrm{~d}$

$6 \mathrm{~d}$

31d $-4 d$

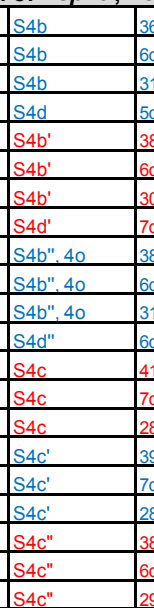

\begin{tabular}{|l|l|}
\hline $36 d$ \\
\hline $6 d$ \\
\hline $31 d$ \\
\hline $5 d$ \\
\hline $38 d$ \\
\hline $6 d$ \\
\hline $30 d$ \\
\hline $7 d$ \\
\hline $38 d$ \\
\hline $6 d$ \\
\hline $31 d$ \\
\hline $6 d$ \\
\hline $41 d$ \\
\hline $7 d$ \\
\hline $28 d$ \\
\hline $39 d$ \\
\hline $7 d$ \\
\hline $28 d$ \\
\hline $38 d$ \\
\hline $6 d$ \\
\hline $29 d$ \\
\hline
\end{tabular}

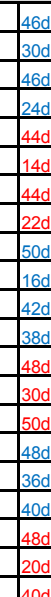

Lifespan Assays of human Ab42 ${ }^{\text {E693G }}$ expressing flies with expression of Smo ${ }^{\text {Act }}$ and $\mathrm{Hsp68}$ in the glia

control:UAS:GFP NLS/Repo-GAL4

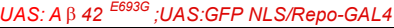

UAS: A $\beta 422^{\text {E693G }}$ :UAS:Smo ${ }^{\text {Act }}$ Repo-GAL4

UAS: $A \beta 42^{\text {E693G }} ;$ UAS:Hsp $68 / R e p o-G A L 4$

Control: UAS:GFP NLS/Repo-GAL4

UAS:A $42^{\text {E693G }}$,UAS:GFP NLS/Repo-GAL4

UAS: A $\beta 422^{\text {E693G } ; U A S: S m o}{ }^{\text {Act }}$ Repo-GAL4

UAS:A $342^{\text {E693G }}$ UAS:Hsp68/Repo-GAL4

\begin{tabular}{|l|l|l|}
\hline Figure & $20 \%$ survival time & N \\
\hline $5 \mathrm{a}, 5 \mathrm{~b}$ & $32 \mathrm{~d}$ & $48 \mathrm{~d}$ \\
\hline $5 \mathrm{a}, 5 \mathrm{~b}$ & $22 \mathrm{~d}$ & $28 \mathrm{~d}$ \\
\hline $5 \mathrm{a}$ & $26.3 \mathrm{~d}$ & $30 d$ \\
\hline $5 \mathrm{~b}$ & $26 \mathrm{~d}$ & $32 d$ \\
\hline Table S2 & $24.5 \mathrm{~d}$ & $46 \mathrm{~d}$ \\
\hline Table S2 & $16.2 \mathrm{~d}$ & $32 d$ \\
\hline Table S2 & $20.2 \mathrm{~d}$ & $42 d$ \\
\hline
\end{tabular}

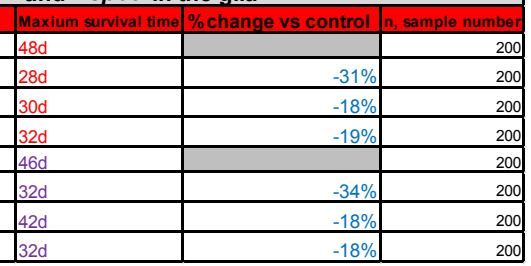




\section{Supplementary Table 3: Summary of Climbing ability}

bioRxiv preprint doi: https://doi.org/10.1101/2020.02.05.935585; this version posted February 6, 2020. The copyright holder for this preprint

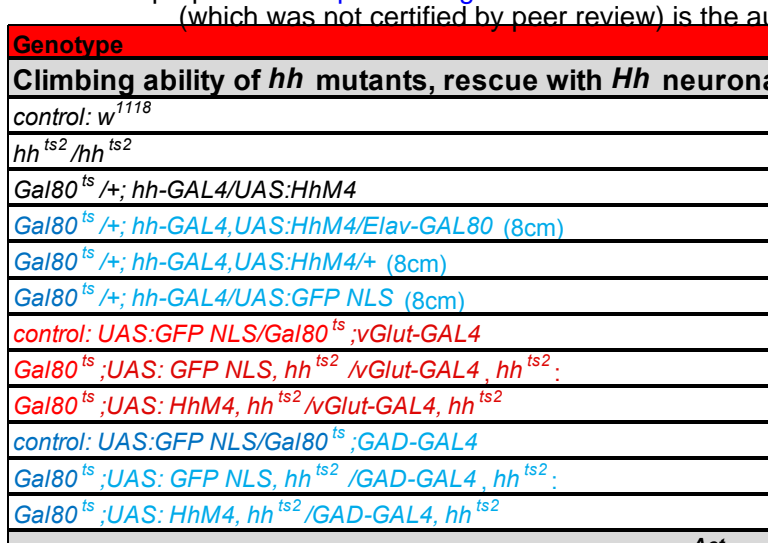

\begin{tabular}{|c|c|c|c|}
\hline \multirow{2}{*}{\multicolumn{2}{|c|}{\begin{tabular}{l|l} 
Figure & 0 Day climbing ability \\
I expression $\&$ H OE flies
\end{tabular}}} & Aged climbing ability & \multirow[t]{2}{*}{ Two-way Anova test } \\
\hline & & & \\
\hline $2 \mathrm{~b}$ & $93 \pm 0.8 \% ; 83 \pm 1.4 \%(5 d)$ & $66 \pm 2.2 \%(15 d)$ & \\
\hline $2 \mathrm{~b}$ & $86 \pm 1.8 \% ; 30 \pm 2.7(5 \mathrm{~d})$ & $4 \pm 1.1 \%(15 d)$ & $<0.001^{* \star *}$ \\
\hline $2 \mathrm{~b}$ & $97 \pm 0.8 \% ; 95 \pm 1.0(5 d)$ & $88 \pm 1.6 \%(15 d)$ & $<0.001^{* * *}$ \\
\hline $2 \mathrm{~d}$ & $96+1.1 \%$ & $20 \pm 1.5 \%(35 d)$ & $<0.001^{\star \star *}$ \\
\hline $\mathrm{d}$ & $96 \pm 1.0 \%$ & $39 \pm 1.7 \%(35 d)$ & \\
\hline $2 \mathrm{~d}$ & $90 \pm 1.7 \%$ & $19 \pm 2.1 \%(35 d)$ & $<0.001^{* * *}$ \\
\hline $2 f$ & $100 \pm 0 \%$ & $70 \pm 2.6 \%(20 d)$ & $<0.001^{* \star *}$ \\
\hline$\underline{2 f}$ & $84 \pm 1.8 \%$ & $26 \pm 2.3 \%(20 d)$ & $<0.001^{* * *}$ \\
\hline $2 f$ & $93 \pm 1.3 \%$ & $55 \pm 2.0 \%(20 \mathrm{~d})$ & \\
\hline$S 3 b$ & $100 \pm 0.3 \%$ & $63 \pm 3.4 \%(20 d)$ & $<0.001^{* \star *}$ \\
\hline$S 3 b$ & $88 \pm 1.5 \%$ & $22 \pm 2.4 \%(20 d)$ & \\
\hline $\mathrm{S} 3 \mathrm{~b}$ & $84 \pm 1.2 \%$ & $45 \pm 2.2 \%(20 d)$ & $<0.001^{\star \star *}$ \\
\hline & & & \\
\hline & $100 \pm 0 \%$ & $77 \pm 2.2 \%(20 d)$ & $<0.001^{* * *}$ \\
\hline $3 d$ & $89 \pm 1.4 \%$ & $17 \pm 1.8 \%(20 \mathrm{~d})$ & \\
\hline $3 d$ & $89 \pm 1.2 \%$ & $54 \pm 2.9 \%(20 d)$ & $<0.001^{\text {*** }}$ \\
\hline the $g$ & & & \\
\hline $4 \mathrm{p}$ & $94 \pm 1.2 \%$ & $66 \pm 1.8 \%(15 d)$ & $<0.001^{* * *}$ \\
\hline $4 p$ & $82 \pm 1.0 \%$ & $10 \pm 1.6 \%(15 d)$ & \\
\hline $4 \mathrm{p}$ & $80 \pm 1.7 \%$ & $38 \pm 1.5 \%(15 d)$ & $<0.001^{* * *}$ \\
\hline ith ex & ion of Smo ${ }^{A c t}$ an & Hsp68 in the glia & \\
\hline $5 c$ & $95 \pm 1.0 \%$ & $73 \pm 2.3 \%(14 d)$ & $<0.001^{\text {*** }}$ \\
\hline $5 c$ & $82 \pm 1.9 \%$ & $21 \pm 2.7 \%(14 \mathrm{~d})$ & \\
\hline & $89 \pm 1.3 \%$ & $56 \pm 1.8 \%(14 d)$ & $<0.001^{\star * \star *}$ \\
\hline & $77 \pm 1.2 \%$ & $66 \pm 1.6 \%(14 d)$ & $<0.001^{* \star *}$ \\
\hline
\end{tabular}




\section{Explanation of genotypes}

\section{genotype}

ci-Gal4; UAS GFP NLS

$w^{1118}$; UAS:GFP NLS ; ci-

Gal4 Trojan

\section{Figure 1b-b"}

UAS:mCD8::GFP; repo-

Gal4

$w^{1118} ;$ UAS:mCD8 ::GFP/rep

o-Gal4

Figure 1c-c', S1b-b", S5g-

h"; S5k-n"

UAS:mCD8::GFP; $\boldsymbol{h} \boldsymbol{h}-\mathrm{Gal} \mathbf{4}$ $w^{1118 ;}$ UAS: $m C D 8:: G F P / h h-$ Gal4

Expression of GFP-NLS in $\mathrm{Ci}$ expressing cells

Repo-

Gal4>mCD8::GFP
hh-Gal4>

mCD8::GF

$P$

\section{Figure 1e-e'";}

UAS:GFP; $h h-G a l 4$

$w^{1118}$; UAS:GFP/hh-

T2AGal4 (Trojan)

\section{Figure S1g-g"';}

\section{hh-Gal4 (control)}

$w^{1118} ; \mathrm{Gal} 80^{t s} /+; h h-$

GAL4/UAS: GFP NLS

\begin{tabular}{l|l} 
Gal80 $^{\text {ts }}$ & $\begin{array}{l}\text { Gal4>GF } \\
\text { P NLS }\end{array}$
\end{tabular}

hh-Gal4>

GFP

$P$ NLS
Expression of a membrane

bound

mCD8::GFP, specifically in glia

Expression of a membrane bound mCD8::GFP, specifically in hh expressing cells

Expression of a cytoplasmic GFP, specifically in hh expressing cells

Overexpression of GFP NLS (neutral transgene) in hh expressing cells using the UAS Gal4 binary system and the thermosensitive variant of Gal80, repressor of Gal4. 


\section{Explanation of genotypes}

\section{Control}

$w^{1118}$; UAS:GFP::NLS/ddc-

Gal4

Figure 1g-g", 1i-j, S2f-f"'

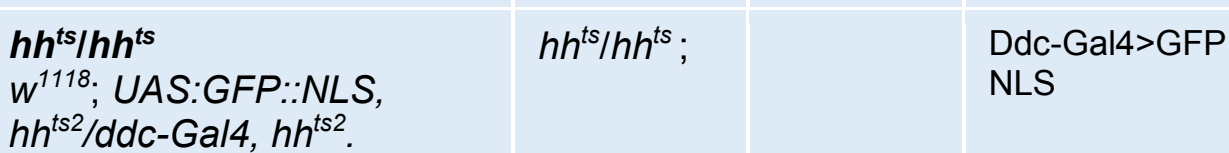

Figure 1h-h", 1i-1i, S2g-g""

mCD8::GFP/ddc-Gal4

$W^{1118}$;

UAS :mCD8 ::GFP/ddc-

Gal4

Figure 1k-l, 1o S2a-b, S2e
$h h^{t s} / h h^{t s}$;
mCD8::GFP/ddc-Gal4
$w^{1118}$; UAS :mCD8 $:: G F P$,
$h h^{t s} / h h^{t s}$
Ddc-
Gal4>mCD8::GF
$\mathrm{P}$

$h h^{t s 2} / d d c-G a l 4, h h^{t s 2}$.

Figure 1m-n, 10, S2c-d, $\underline{\text { S2e }}$

Ddc-

Gal4>mCD8::GF

$\mathrm{P}$
Expression of a nuclear GFP, specifically in Dopaminergic neurons

Expression of a nuclear GFP, specifically in Dopaminergic neurons in a $h h^{t s 2} / h h^{t s 2}$ mutant background.

Expression of a membrane bound mCD8::GFP, specifically in Dopaminergic neurons

Expression of a membrane bound mCD8::GFP, specifically in Dopaminergic neurons in a $h h^{t s 2} / h h^{t s 2}$ mutant background.

fly strain with $w^{1118}$ control genetic background

A hh temperature sensitive allele $\left(h h^{t s 2}\right)$, at permissive temperature $\left(18^{\circ} \mathrm{C}\right)$ is inactive, at

Figure 2a-b, S3c 


\section{Explanation of genotypes}

\begin{tabular}{|c|c|c|c|}
\hline & & & $\begin{array}{l}\text { non-permissive } \\
\text { temperature } \\
\left(29^{\circ}\right) \text {, is } \\
\text { considered a } \\
h h \text { null. }\end{array}$ \\
\hline $\begin{array}{l}\boldsymbol{h}^{\boldsymbol{h}^{t s}} / \boldsymbol{h} \boldsymbol{h}^{15} \text { mutant } \\
w^{1118} ; h h^{t s 2} / h h^{15} \\
\text { Figure 2a }\end{array}$ & $h h^{t s 2} / h h^{15}$ & & $\begin{array}{l}\text { A heteroallelic } \\
\text { combination of } \\
\text { the } h h \\
\text { temperature } \\
\text { sensitive allele } \\
\left(h h^{t s 2}\right) \text { and the } \\
\text { null allele } h h^{15} \text {. }\end{array}$ \\
\hline $\begin{array}{l}\text { hh-Gal4 (control) } \\
w^{1118} ; \text { Gal80 } /+; h h- \\
\text { GAL4/UAS:GFP RNAi } \\
\text { Figure 2a, }\end{array}$ & Gal $80^{\text {ts }}$ & $\begin{array}{l}\text { Gal4>GFP } \\
\text { RNAi }\end{array}$ & $\begin{array}{l}\text { Overexpression } \\
\text { of GFP RNAi } \\
\text { (neutral RNAi) } \\
\text { in } h h \text { expressing } \\
\text { cells using the } \\
\text { UAS Gal4 binary } \\
\text { system and the } \\
\text { thermosensitive } \\
\text { variant of Gal80, } \\
\text { repressor of } \\
\text { Gal4. }\end{array}$ \\
\hline $\begin{array}{l}\text { hh OE (hh-Gal4): } \\
\text { w }^{1118 ;} \text { Gal80 } / \mathrm{ts}+\text {; } h \text {-Gal4, } \\
\text { UAS:HhM4/UAS:GFP RNAi } \\
\text { Figure 2a-b }\end{array}$ & Gal80ts & $\begin{array}{l}\text { Gal4>Hh } \\
\text { M4 }\end{array}$ & $\begin{array}{l}\text { Overexpression } \\
\text { of } h h \text { in } h h- \\
\text { expressing } \\
\text { cells using the } \\
\text { UAS Gal4 } \\
\text { binary system } \\
\text { and the } \\
\text { thermosensitive } \\
\text { variant of } \\
\text { Gal80, } \\
\text { repressor of } \\
\text { Gal4. At } 29^{\circ} \mathrm{C} \text {, } \\
\text { Gal80 activity is } \\
\text { abolished, } \\
\text { leading to the } \\
\text { full activity of } \\
\text { Gal4 under } h h \\
\text { promoter }\end{array}$ \\
\hline
\end{tabular}




\section{Explanation of genotypes}

\begin{tabular}{|c|c|c|c|c|c|}
\hline $\begin{array}{l}\text { hh OE (hh-Gal4): } \\
w^{1118 ;} \text { Gal } 80^{t s} /+; h h \\
\text { Gal4,UAS:HhM4/UAS:GFP } \\
\text { NLS } \\
\text { Figure 2c-d, S5t-u }\end{array}$ & Gal $80^{\text {ts }}$ & $\begin{array}{l}\text { Gal4>Hh } \\
\text { M4 }\end{array}$ & & & $\begin{array}{l}\text { See above - } \\
h h \text { OE (hh- } \\
\text { Gal4): } \\
\text { explanation }\end{array}$ \\
\hline $\begin{array}{l}\text { hh OE + elav-Gal80: } \\
\text { w }^{1118} ; \text { Gal } 80^{\text {ts }} / \text { elav-Gal80; } \\
\text { hh-GAL4,UAS:HhM4/+ } \\
\text { Figure 2c-d }\end{array}$ & Gal80 ${ }^{\text {ts }}$ & $\begin{array}{l}\text { Gal4>Hh } \\
\text { M4 }\end{array}$ & Gal80 & & $\begin{array}{l}\text { Hh OE with } \\
\text { constitutive } \\
\text { expression of } \\
\text { the Gal80 } \\
\text { repressor } \\
\text { which } \\
\text { represses } \\
\text { expression of } \\
\text { Gal4 and thus } \\
\text { the UAS Hh } \\
\text { transgene in all } \\
\text { Elav positive } \\
\text { neurons. }\end{array}$ \\
\hline 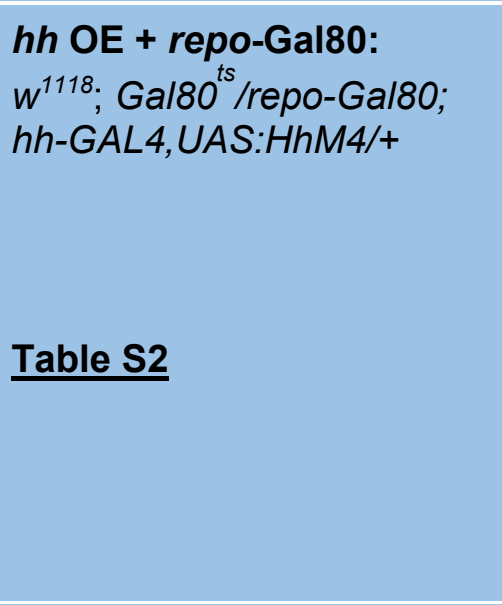 & Gal80 & $\begin{array}{l}\text { Gal4>Hh } \\
\text { M4 }\end{array}$ & & Gal80 & $\begin{array}{l}\text { Hh OE with } \\
\text { constitutive } \\
\text { expression of } \\
\text { the Gal80 } \\
\text { repressor } \\
\text { which } \\
\text { represses } \\
\text { expression of } \\
\text { Gal4 and thus } \\
\text { the UAS Hh } \\
\text { transgene in all } \\
\text { Repo positive } \\
\text { glia. }\end{array}$ \\
\hline $\begin{array}{l}\text { hh mutant + hh OE (vGlut- } \\
\text { Gal4) } \\
w^{11118} ; \text { Gal80 ts. UAS: HhM4, } \\
h h^{t s 2} / V G / u t-G A L 4, h h^{t s 2} \\
\text { Figure 2e-f }\end{array}$ & $\begin{array}{l}\text { Gal } 80^{\text {ts }} \\
h h^{t s 2} / h h^{t s 2}\end{array}$ & & $\begin{array}{l}\text { vGlut- } \\
\text { Gal4>HhM4 }\end{array}$ & & $\begin{array}{l}\text { Overexpression } \\
\text { of } h h \text { in } \\
\text { glutamatergic } \\
\text { neurons in a } \\
h h^{t s 2} / h h^{t s 2} \\
\text { mutant } \\
\text { background. }\end{array}$ \\
\hline $\begin{array}{l}\text { hh mutant (vGlut-Gal4) } \\
w^{1118} ; \text { Galdo } 0^{t s} ; U A S: G F P \\
N L S, h h^{t s 2} / V G / u t-G A L 4, h h^{t s 2} \\
\text { Figure 2e-f }\end{array}$ & $\begin{array}{l}\text { Gal } 80^{\text {ts }} \\
h h^{t s 2} / h h^{t s 2}\end{array}$ & & $\begin{array}{l}\text { vGlut Gal4>GFP } \\
\text { NLS }\end{array}$ & & $\begin{array}{l}\text { Overexpression } \\
\text { of GFP-NLS in } \\
\text { glutamatergic } \\
\text { neurons in a } \\
h h^{t s 2} / h h^{t s 2}\end{array}$ \\
\hline
\end{tabular}




\section{Explanation of genotypes}

\begin{tabular}{|c|c|c|c|c|}
\hline & & & & $\begin{array}{l}\text { mutant } \\
\text { background. }\end{array}$ \\
\hline $\begin{array}{l}\text { vGlut-Gal4 (control) } \\
\text { w }^{1118 ;} \text { Gal80 ts ; UAS:GFP } \\
\text { NLS/vGlut-GAL4 } \\
\text { Figure 2e-f }\end{array}$ & Gal80 ts & $\begin{array}{l}\text { vGlut Gal4>GFP } \\
\text { NLS }\end{array}$ & & $\begin{array}{l}\text { Overexpression } \\
\text { of GFP NLS in } \\
\text { glutamatergic } \\
\text { neurons }\end{array}$ \\
\hline 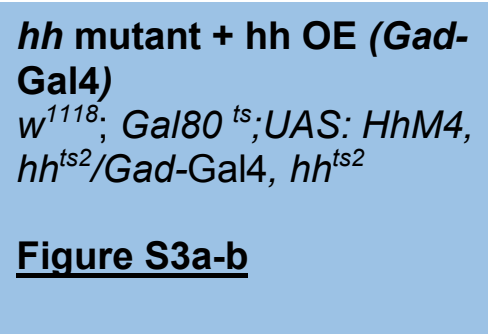 & $\begin{array}{l}\text { Gal80 ts } \\
h h^{t s 2} / h h^{t s 2}\end{array}$ & Gad Gal4>HhM4 & & $\begin{array}{l}\text { Overexpression } \\
\text { of } h h \text { in } \\
\text { GABAergic } \\
\text { neurons in a } \\
h h^{t s 2} / h h^{t s 2} \\
\text { mutant } \\
\text { background. }\end{array}$ \\
\hline $\begin{array}{l}\text { hh mutant (Gad-Gal4) } \\
\text { w }^{1118 ;} \text { Gal80 }{ }^{t s} ; \text { UAS:GFP } \\
\text { NLS, } h h^{t s 2} / G a d-G a l 4, h h^{t s 2} \\
\text { Figure S3a-b }\end{array}$ & $\begin{array}{l}\text { Gal80 ts } \\
h h^{t s 2} / h h^{t s 2}\end{array}$ & $\begin{array}{l}\text { Gad Gal4>GFP } \\
\text { NLS }\end{array}$ & & $\begin{array}{l}\text { Overexpression } \\
\text { of GFP NLS in } \\
\text { GABAergic } \\
\text { neurons in a } \\
h h^{t s 2} / h h^{t s 2} \\
\text { mutant } \\
\text { background. }\end{array}$ \\
\hline $\begin{array}{l}\text { Gad-Gal4 (control) } \\
\text { w }^{1118 ;} \text { Gal80 }{ }^{t s} ; \text { UAS:GFP- } \\
\text { NLS/Gad-Gal4 } \\
\text { Figure S3a-b }\end{array}$ & Gal80 ts & $\begin{array}{l}\text { Gad Gal4>GFP } \\
\text { NLS }\end{array}$ & & $\begin{array}{l}\text { Overexpression } \\
\text { of GFP NLS in } \\
\text { GABAergic } \\
\text { neurons }\end{array}$ \\
\hline $\begin{array}{l}\text { RNAi ci (repo-Gal4) } \\
\text { w }^{1118 ;} \text {; UAS: Dcr2 /Gal80 ts; } \\
\text { UAS: Ci RNAi /Repo-Gal4 } \\
\text { Figure 3a, S1e-e" }\end{array}$ & Gal80 ts & & $\begin{array}{l}\text { Repo-Gal4> RNAi } \\
\text { ci }\end{array}$ & $\begin{array}{l}\text { Glial-specific } \\
\text { RNAi } \\
\text { knockdown of } \\
\text { ci }\end{array}$ \\
\hline $\begin{array}{l}\text { RNAi smo (repo-Gal4) } \\
\text { w }^{1118 ;} \text {; UAS: smo RNAi/+; } \\
\text { smo }{ }^{42} \text {, UAS: Dcr2/Gal80"s. } \\
\text { Repo-GAL4/+ } \\
\text { Figure 3a, S1c-c" }\end{array}$ & $\begin{array}{l}\text { Gal80 } \\
\text { smo }^{\text {ts }}\end{array}$ & & $\begin{array}{l}\text { Repo-Gal4> RNAi } \\
\text { smo }\end{array}$ & $\begin{array}{l}\text { Glial-specific } \\
\text { RNAi } \\
\text { knockdown of } \\
\text { smo in a } \\
\text { smo } 442 \\
\text { heterozygote } \\
\text { mutant } \\
\text { background. }\end{array}$ \\
\hline control (repo-Gal4) & Gal80 ts & & $\begin{array}{l}\text { Repo-Gal4> RNAi } \\
\text { GFP }\end{array}$ & $\begin{array}{l}\text { Glial-specific } \\
\text { knockdown of }\end{array}$ \\
\hline
\end{tabular}




\title{
Explanation of genotypes
}

$w^{1118} ;$ UAS:GFP RNAi/

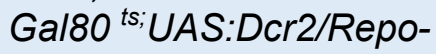

GAL4

\section{Figure 3a}

\section{RNAi ci (elav-Gal4)}

$w^{1118}$, elav-Gal4/+; UAS:

Dcr2/Gal80 ${ }^{\text {ts. }}$ UAS:ci RNAi/+

\section{Figure 3b}

\section{RNAi smo (elav-Gal4)}

$w^{1118}$, UAS: smo RNAilelav

Gal4; smo ${ }^{X 42}$,

UAS:Dcr2/Gal80 ${ }^{\text {ts }}$

\section{Figure $3 b$}

\section{control (elav-Gal4)}

$w^{1118}$, elav-

Gal4/+;UAS:Dcr2 UAS:GFP

RNAi/Gal80 $0^{\text {ts }}$

\section{Figure $3 b$}

$c i{ }^{\text {act }}$ OE (repo-Gal4) in $h h$ mutant

$w^{1118 ;}$ Gal80 ${ }^{\text {ts. }}$ UAS: $i^{\text {act }}$,

$h h^{t s 2} / r e p o-G a l 4, h h^{t s 2}$

Figure 3c-d, 3i, 3l-o, 4b

\section{S3k S4a-a"}

\section{hh mutant (repo-Gal4)}

w $^{1118 ;}$ Gal80 ts; UAS:GFP

$N L S, h h^{t s 2} / r e p o-G a l 4, h h^{t s 2}$

Figure 3c-d, 3h, 3k, 3m-o,

4b, 4e-f, 4h, 4l-p S3k S4a-

d", S5S

control (repo-Gal4)

w $^{1118 ;}$; UAS:GFP NLS/ Gal80

ts; Repo-GAL4

\author{
Gal80 ts; \\ Elav-Gal4> \\ RNAi ci
}

Gal80 ${ }^{\text {ts }}$;

$s m 0^{X 42}$

Elav-Gal4>

RNAi smo

control GFP

RNAi

Neuron-specific RNAi knockdown of ci

Neuron-specific RNAi

knockdown of smo in a $s m o^{\mathrm{X} 42}$ heterozygote mutant background.

Gal80 ts;

Elav-Gal4>

RNAi GFP

Gal80 ts ;

$h h^{t s 2} / h h^{t s 2}$

Repo-Gal4> ${ }^{\text {act }}$

Overexpression of an activated form of $c i\left(c i^{\text {act }}\right)$ in glia in

$h h^{t s 2} / h h^{t s 2}$

mutant background.

Gal80 ts:

$h h^{t s 2} / h h^{t s 2}$

Gal80 ts
Repo-Gal4> GFP NLS

Repo-Gal4> GFP NLS
Overexpression of GFP-NLS in glia in $h h^{t s 2} / h h^{t s 2}$ mutant background.

Overexpression of GFP-NLS in glia 


\section{Explanation of genotypes}

Figure 3c-g, 3j, 3m-o, 4b, 4q-g', 4i 4l-p, 5a-c, 5e-e"”, 5i-m, S3k S4a-d", S5r-s, S6b-b"', S6d-d"', S6f-f'", S6h-h"'

ci ${ }^{\text {act }}$ OE (repo-Gal4)

$w^{1118 ;}$ Gal80 ts; UAS:ci

act/repo-Gal4

\section{Figure 3e}

smo act OE (repo-Gal4)

W $^{1118 ;}$ Gal80 ts. UAS:smo

act/repo-Gal4

\section{Figure $3 f$}

16xLexAop2-

mCD8GFP/5x mCD8-

Cherry; ci-LexA/repo-Gal4

$w^{1118}, 16 x$ LexAop2-

mCD8GFP/5xmCD8-

Cherry; ci-LexA/repo-Gal4

\section{Figure S1a-a"}

\section{Gal80 ts}

Repo-Gal4> ci ${ }^{\text {act }}$

\section{Gal80 ts}

Repo-Gal4> smo act

RepoGal4>mCD8::Ch erry

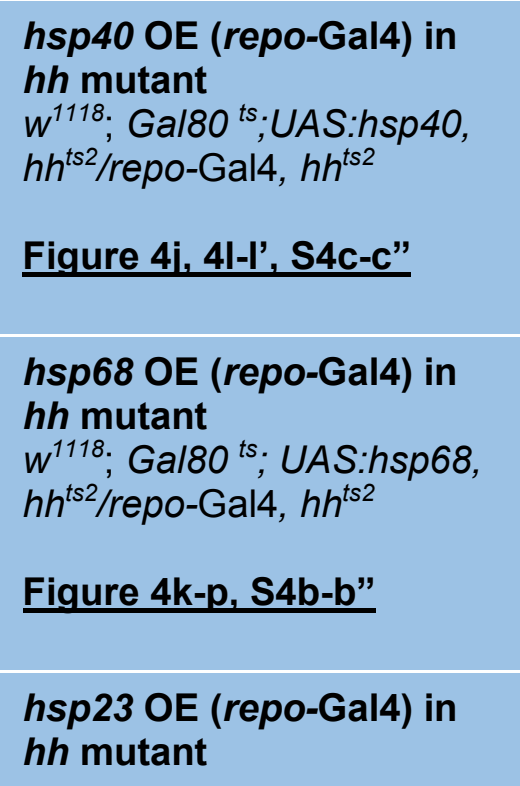

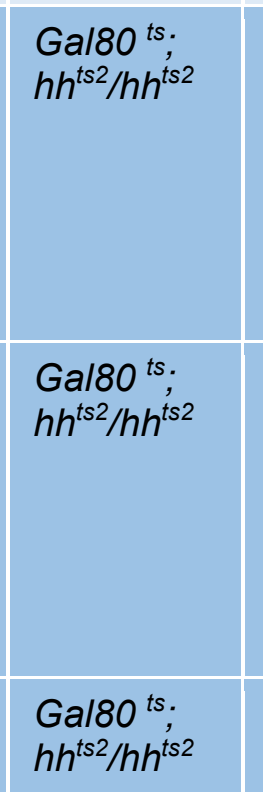

Repo-Gal4> hsp40

Or

Overexpres
of an activ
form of ci
in glia
Overexpress
of an acti
form of sm
(smo act)
Expressio
membran
bound
mCD8::Ch
specifically
glia (repo-
along with
expressio
mCD8GF
Ci expres
cells (using
exAop-lex
Overexpres
(stem) of $h s p 40$ in glia in $h h^{t s 2} / h h^{t s 2}$ mutant background.

Repo-Gal4> hsp68

Overexpression of hsp68 in glia in $h h^{t s 2} / h h^{t s 2}$ mutant background.

Repo-Gal4> hsp23

Overexpression of $h s p 23$ in glia 


\section{Explanation of genotypes}

\begin{tabular}{|c|c|c|c|}
\hline $\begin{array}{l}w^{1118 ;} \text { Gal80 }{ }^{t s} ; \text { UAS:hsp23, } \\
h h^{t s 2} / \text { repo-Gal4, } h h^{t s 2} \\
\text { Figure S4d-d" }\end{array}$ & & & $\begin{array}{l}\text { in } h h^{t s 2} / h h^{t s 2} \\
\text { mutant } \\
\text { background. }\end{array}$ \\
\hline 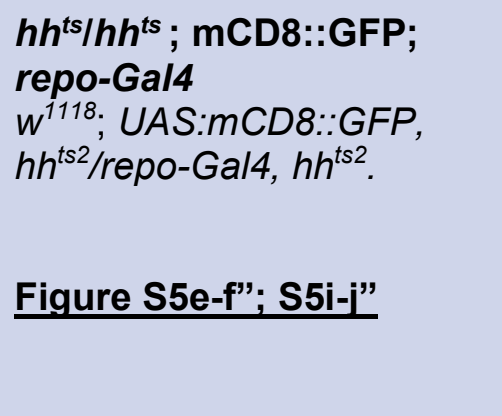 & $h h^{t s 2} / h h^{t s 2}$ & $\begin{array}{l}\text { Repo- } \\
\text { Gal4>mCD8::GFP }\end{array}$ & $\begin{array}{l}\text { Expression of a } \\
\text { membranous } \\
\text { bound } \\
\text { mCD8::GFP, } \\
\text { specifically in } \\
\text { glia in a } \\
h h^{t s 2} / h h^{t s 2} \\
\text { mutant } \\
\text { background. }\end{array}$ \\
\hline $\begin{array}{l}\text { ci-Gal4; mCD8-GFP } \\
\text { w}^{1118 ;} \text { UAS:mCD8 GFP; } \\
\text { ci-Gal4 Trojan } \\
\text { Figure S5o-p", }\end{array}$ & & & $\begin{array}{l}\text { Expression of a } \\
\text { membrane } \\
\text { bound } \\
\text { mCD8::GFP } \\
\text { specifically in } c i \\
\text { expressing } \\
\text { cells }\end{array}$ \\
\hline 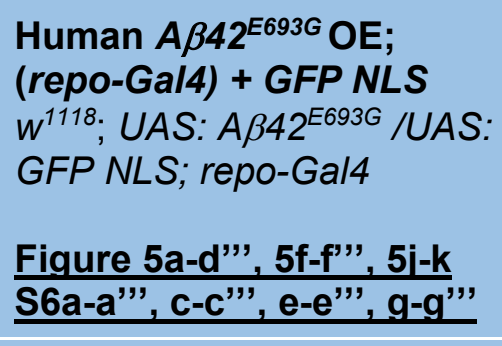 & & $\begin{array}{l}\text { Repo-Gal4> } \\
A \beta 42^{E 693 G}\end{array}$ & $\begin{array}{l}\text { Overexpressio } \\
\mathrm{n} \text { of human } \\
\text { artic variant } \\
\text { A } 342^{E 693 G} \text { and } \\
\text { GFP NLS in } \\
\text { glia }\end{array}$ \\
\hline 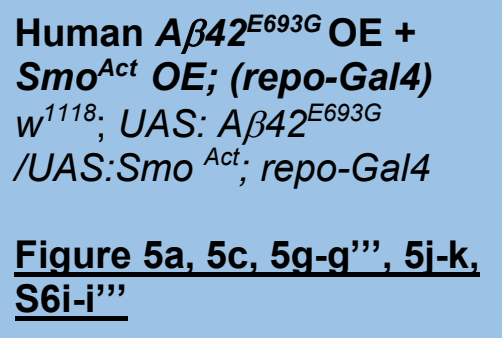 & & $\begin{array}{l}\text { Repo-Gal4> } \\
\text { A } \beta 42^{E 693 G} / \text { Smo } \\
\text { Act }\end{array}$ & $\begin{array}{l}\text { Overexpressio } \\
\mathrm{n} \text { of human } \\
\text { artic variant } \\
A \beta 42^{E 693 G} \text { and } \\
S m 0^{A c t} \text { in glia }\end{array}$ \\
\hline $\begin{array}{l}\text { Human } A \beta 42^{E 693 G} \text { OE; } \\
\text { (repo-Gal4) + Hsp68 } \\
\text { W }^{1118 ;} \text { UAS: A } A 42^{E 693 G} \\
\text {;UAS:Hsp68/ repo-Gal4 } \\
\text { Figure 5b-c, h-h"”, 5j-k, } \\
\text { S6j-j"'” }\end{array}$ & & $\begin{array}{l}\text { Repo-Gal4> } \\
A \beta 42^{E 693 G ; h s p 68}\end{array}$ & $\begin{array}{l}\text { Overexpressio } \\
\mathrm{n} \text { of human } \\
\text { artic variant } \\
A \beta 42^{E 693 G} \text { and } \\
\text { hsp68 in glia }\end{array}$ \\
\hline
\end{tabular}


bioRxiv preprint doi: https://doi.org/10.1101/2020.02.05.935585; this version posted February 6, 2020. The copyright holder for this preprint (which was not certified by peer review) is the author/funder. All rights reserved. No reuse allowed without permission.

\section{Table S4. $\quad$ Explanation of genotypes}

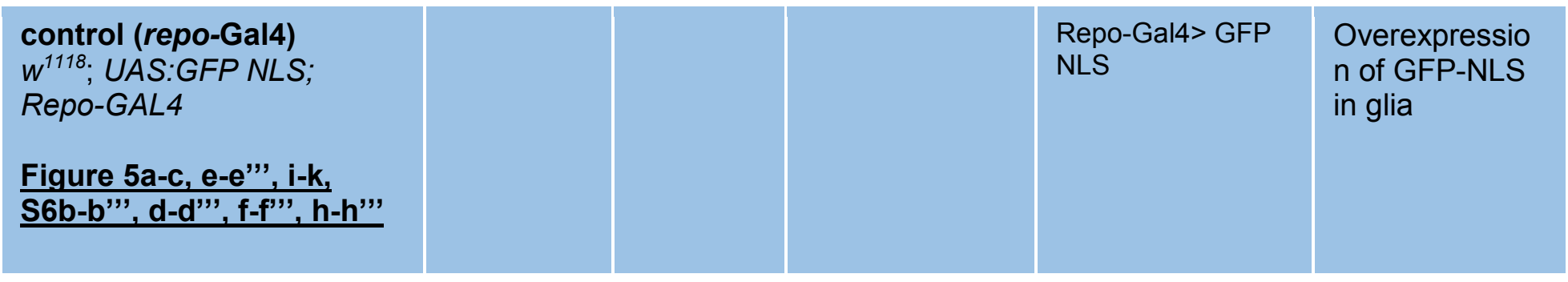


Table S5. Statistics for Lifespan Assays

bioRxiv preprint doi: https://doi.org/10.1101/2020.02.05.935585; this version posted February 6, 2020. The copyright holder for this preprint (which was not certified by peer review) is the author/funder. All rights reserved. No reuse allowed without permission.

\begin{tabular}{|c|c|c|c|c|c|}
\hline \multicolumn{2}{|c|}{ Data Set 1} & \multicolumn{2}{|c|}{ Control Data Set 2} & \multirow[b]{2}{*}{$P$ value } & \multirow[b]{2}{*}{ Figure } \\
\hline Genotype & $\begin{array}{l}\text { Median } \\
\text { Survival } \\
\text { Time }\end{array}$ & Genotype & $\begin{array}{l}\text { Median } \\
\text { Survival } \\
\text { Time }\end{array}$ & & \\
\hline hh OE (hh-Gal4) & $48 d$ & hh-Gal4 (control) & $38 d$ & $P<0.0001$ & $2 a$ \\
\hline$h h^{t s} / h h^{t s}$ mutant & $8 d$ & $w^{1118}$ (control) & $38 d$ & $P<0.0001$ & $2 a$ \\
\hline$h h^{t s} / h h^{15}$ mutant & $6 d$ & $w^{1118}$ (control) & $38 d$ & $P<0.0001$ & $2 a$ \\
\hline hh OE + elav-Gal80: & $38 d$ & hh OE (hh-Gal4) & $43 d$ & $P<0.0001$ & $2 c$ \\
\hline$h h$ OE (hh-Gal4) & $43 d$ & hh-Gal4 (control) & $37 d$ & $P<0.0001$ & $2 c$ \\
\hline $\begin{array}{l}\text { hh mutant + hh OE } \\
\text { (vGlut-Gal4) }\end{array}$ & $28 d$ & $\begin{array}{l}\text { hh mutant (vGlut- } \\
\text { Gal4) }\end{array}$ & $7 d$ & $P<0.0001$ & $2 \mathrm{e}$ \\
\hline $\begin{array}{l}\text { hh mutant + hh OE } \\
\text { (Gad-Gal4) }\end{array}$ & $18 d$ & $\begin{array}{l}\text { hh mutant (Gad- } \\
\text { Gal4) }\end{array}$ & $8 d$ & $P<0.0001$ & S3a \\
\hline $\begin{array}{l}\text { RNAi smo (repo- } \\
\text { Gal4) }\end{array}$ & $25 d$ & control (repo-Gal4) & $44 d$ & $P<0.0001$ & $3 a$ \\
\hline RNAi ci (repo-Gal4) & $23 d$ & control (repo-Gal4) & $44 d$ & $P<0.0001$ & $3 a$ \\
\hline $\begin{array}{l}C i^{\text {act }} \text { OE (repo-Gal4) } \\
\text { in } h h \text { mutant }\end{array}$ & $31 d$ & $\begin{array}{l}\text { hh mutant (repo- } \\
\text { Gal4) }\end{array}$ & $6 d$ & $P<0.0001$ & $3 c$ \\
\hline $\begin{array}{l}\text { hsp40 OE (repo- } \\
\text { Gal4) in hh mutant }\end{array}$ & $28 d$ & $\begin{array}{l}\text { hh mutant (repo- } \\
\text { Gal4) }\end{array}$ & $7 d$ & $P<0.0001$ & S4C \\
\hline $\begin{array}{l}\text { hsp68 OE (repo- } \\
\text { Gal4) in hh mutant }\end{array}$ & 31d & $\begin{array}{l}\text { hh mutant (repo- } \\
\text { Gal4) }\end{array}$ & $6 d$ & $P<0.0001$ & $\begin{array}{l}\text { 4o, } \\
\text { S4b" }\end{array}$ \\
\hline$c i^{\text {act }}$ OE (repo-Gal4) & $48 d$ & control (repo-Gal4) & $42 d$ & $P<0.0001$ & $3 e$ \\
\hline $\begin{array}{l}\text { smo act OE (repo- } \\
\text { Gal4) }\end{array}$ & $49 d$ & control (repo-Gal4) & 41d & $P<0.0001$ & $3 f$ \\
\hline $\begin{array}{l}\text { Human } A \beta 42^{E 693 G} \\
\text { OE; (repo-Gal4) }\end{array}$ & $\begin{array}{l}22 d \\
(20 \% \\
\text { survival } \\
\text { time) }\end{array}$ & $\begin{array}{l}\text { Human } A \beta 42^{E 693 G} \\
\text { OE/ Smo }{ }^{A c t} \\
\text { OE;(repo-Gal4) }\end{array}$ & $\begin{array}{l}26 \mathrm{~d} \\
(20 \% \\
\text { survival } \\
\text { time) }\end{array}$ & $P<0.0001$ & $5 a$ \\
\hline $\begin{array}{l}\text { Human } A \beta 42^{E 693 G} \\
\text { OE; (repo-Gal4) }\end{array}$ & $\begin{array}{l}22 d \\
(20 \% \\
\text { survival } \\
\text { time) }\end{array}$ & $\begin{array}{l}\text { Human } A \beta 42^{E 693 G} \\
\text { OE; } h s p 68 \text { OE } \\
\text { (repo-Gal4) }\end{array}$ & $\begin{array}{l}26 \mathrm{~d} \\
(20 \% \\
\text { survival } \\
\text { time) }\end{array}$ & $P<0.0001$ & $5 b$ \\
\hline
\end{tabular}


Table S6.

Statistics of Climbing ability

bioRxiv preprint doi: https://doi.org/10.1101/2020.02.05.935585; this version posted February 6, 2020. The copyright holder for this preprint (which was not certified by peer review) is the author/funder. All rights reserved. No reuse allowed without permission.

\begin{tabular}{|c|c|c|c|c|c|}
\hline \multicolumn{2}{|c|}{ Data Set 1} & \multicolumn{2}{|c|}{ Control Data Set 2} & \multirow[b]{2}{*}{ P value } & \multirow[b]{2}{*}{ Figure } \\
\hline Genotype & $\begin{array}{l}\text { Climbing } \\
\text { activity (\%) }\end{array}$ & Genotype & $\begin{array}{l}\text { Climbing } \\
\text { activity (\%) }\end{array}$ & & \\
\hline hh OE (hh-Gal4) & $88 \%$ & $w^{1118}$ (control) & $66 \%$ & $\mathrm{P}<0.001$ & $2 b$ \\
\hline$h h^{t s} / h h^{t s}$ mutant & $4 \%$ & $w^{1118}$ (control) & $66 \%$ & $P<0.001$ & $2 b$ \\
\hline $\begin{array}{l}\text { hh OE + elav- } \\
\text { Gal80: }\end{array}$ & $20 \%$ & $\begin{array}{l}\text { hh OE (hh- } \\
\text { Gal4) }\end{array}$ & $39 \%$ & $P<0.001$ & $2 d$ \\
\hline $\begin{array}{l}\text { hh mutant + hh } \\
\text { OE (vGlut-Gal4) }\end{array}$ & $55 \%$ & $\begin{array}{l}\text { hh mutant } \\
\text { (vGlut-Gal4) }\end{array}$ & $26 \%$ & $P<0.001$ & $2 f$ \\
\hline $\begin{array}{l}\text { hh mutant + hh } \\
\text { OE (Gad-Gal4) }\end{array}$ & $45 \%$ & $\begin{array}{l}\text { hh mutant } \\
\text { (Gad-Gal4) }\end{array}$ & $22 \%$ & $P<0.001$ & S3b \\
\hline $\begin{array}{l}C i^{\text {act }} \text { OE (repo- } \\
\text { Gal4) in } h h \\
\text { mutant }\end{array}$ & $54 \%$ & $\begin{array}{l}\text { hh mutant } \\
\text { (repo-Gal4) }\end{array}$ & $17 \%$ & $P<0.001$ & $3 d$ \\
\hline $\begin{array}{l}\text { hsp68 OE (repo- } \\
\text { Gal4) in } h h \\
\text { mutant }\end{array}$ & $38 \%$ & $\begin{array}{l}\text { hh mutant } \\
\text { (repo-Gal4) }\end{array}$ & $10 \%$ & $P<0.001$ & $4 p$ \\
\hline $\begin{array}{l}\text { Human } A \beta 42^{E 693 G} \\
\text { OE/ SmoAct OE } \\
\text { (repo-Gal4) }\end{array}$ & $56 \%$ & $\begin{array}{l}\text { Human } \\
A \beta 42^{E 693 G} \text { OE; } \\
\text { (repo-Gal4) }\end{array}$ & $21 \%$ & $P<0.001$ & $5 c$ \\
\hline $\begin{array}{l}\text { Human } A \beta 42^{E 693 G} \\
\text { OE; hsp68 OE } \\
\text { (repo-Gal4) }\end{array}$ & $66 \%$ & $\begin{array}{l}\text { Human } \\
\text { A } 342^{E 693 G} \text { OE; } \\
\text { hsp68 OE } \\
\text { (repo-Gal4) }\end{array}$ & $21 \%$ & $P<0.001$ & $5 c$ \\
\hline
\end{tabular}

